\title{
Advancements in thermoelectric generators for enhanced hybrid photovoltaic system performance
}

\section{Samson Shittu}

Guiqiang $\mathrm{Li}^{\mathrm{a}}$ *

Guiqiang.Li@hull.ac.uk

\section{Yousef Golizadeh Akhlaghi}

Xiaoli Ma

Xudong Zhao ${ }^{\mathrm{a}, * *}$

Xudong.Zhao@hull.ac.uk

Emmanuel

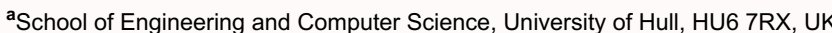

Institute of Robotics, Autonomous Systems and Sensing, University of Leeds, LS2 9JT, UK

*Corresponding author

${ }^{* *}$ Corresponding author

This work is licensed under a Creative Commons Attribution-NonCommercial-NoDerivatives 4.0 International License.

Abstract

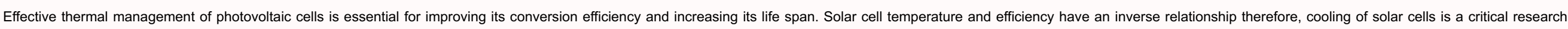

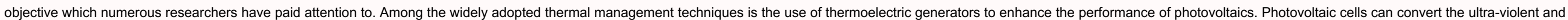

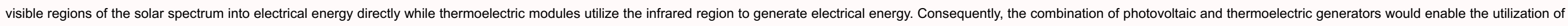

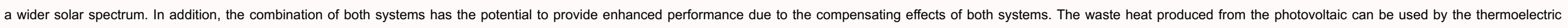

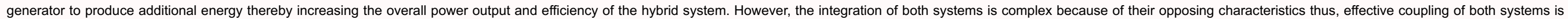

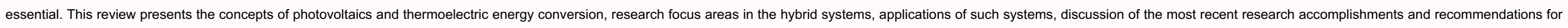
future research. All the essential elements and research areas in hybrid photovoltaic/thermoelectric generator are discussed in detailed therefore, this review would serve as a valuable reference literature.

Keywords: Hybrid photovoltaic system; Thermoelectric generator; Solar energy; Thermal management; Photovoltaic-thermoelectric

\section{Nomenclature}

Fill factor

Temperature, $\mathrm{K}$ 
Solar irradiance, $\mathrm{W} / \mathrm{m}^{2}$

$\mathrm{v}_{\mathrm{oc}}$

Open circuit voltage, $\mathrm{V}$

$\mathrm{I}_{\mathrm{sc}}$

Short circuit current, A

$P_{\text {in }}$

Incident power, W

ZT

Figure of merit

Greek symbols

a

Seebeck coefficient, V/K

$\kappa$

Thermal conductivity, W/m/K

$\sigma$

Electrical conductivity, S/m

$\eta$

Efficiency, \%

Abbreviations

PV

Photovoltaic

TE

Thermoelectric

PV/TEG

Photovoltaic/thermoelectric generator

TPV/TEG 
Cadmium telluride

CIGS

Copper indium gallium selenide

DSSC

Dye-sensitized solar cell

GaAs

Gallium arsenide

GaSb

Gallium antimonide

$\mathrm{CoSb}_{3}$

Copper antimony

InP

Indium phosphide

$\mathrm{Bi}_{2} \mathrm{Te}_{3}$

Bismuth telluride

$\mathrm{PbTe}$

Lead telluride

GeTe

Germanium telluride

SnTe

Tin telluride

I-V

Current-Voltage

TEG

Thermoelectric generator

a-Si

Amorphous silicon 
Lock-On mechanism

\section{Subscripts}

\section{Introduction}

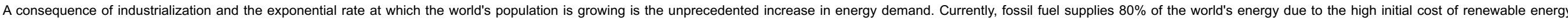

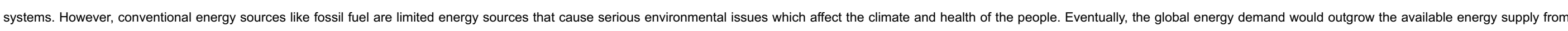

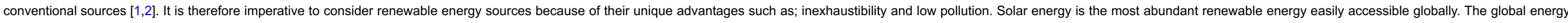
demand can be met by solar energy due to its vast energy capacity. In fact, the total solar radiation impinged on the energy surface is more than 7500 times the world's total annual primary energy consumption of $450 \mathrm{EJ}$ [3].

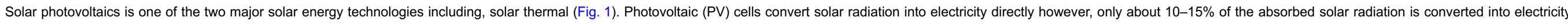

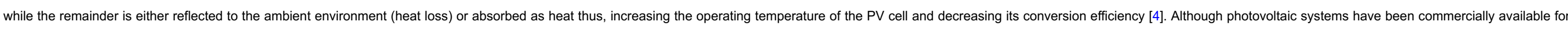

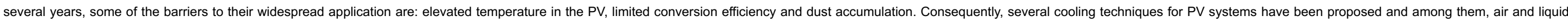

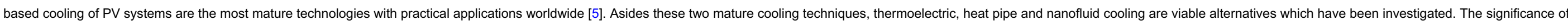

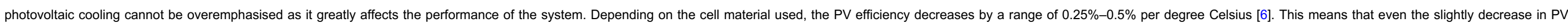




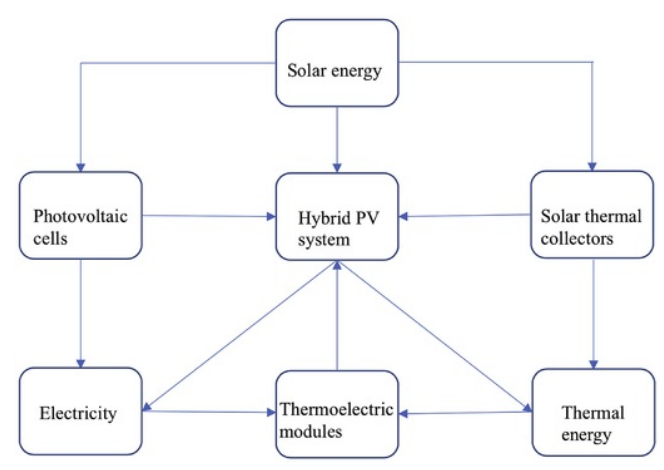

Fig. 1 Solar energy conversion technologies.

\section{alt-text: Fig. 1}

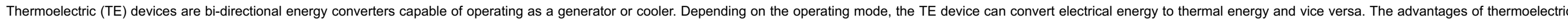

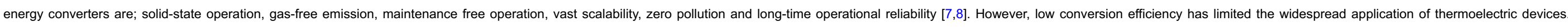

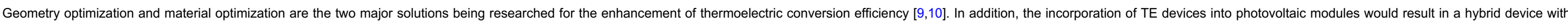

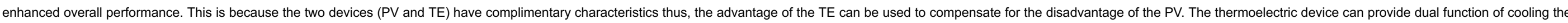
$\mathrm{PV}$ and producing additional energy.

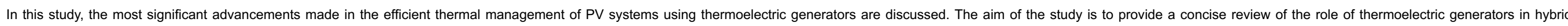

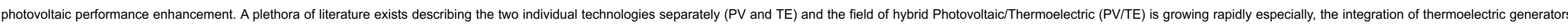

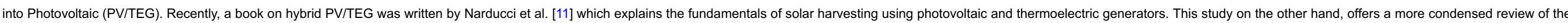

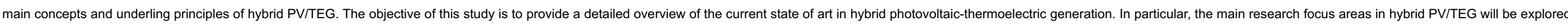

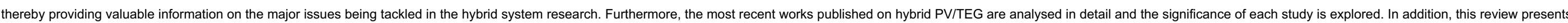

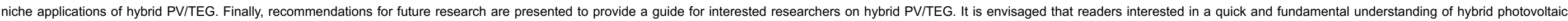

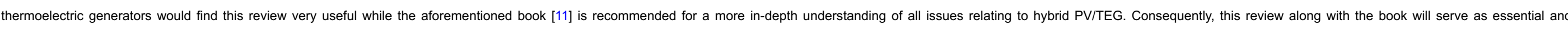
indispensable reference literatures on hybrid PV/TEG.

\section{Photovoltaic systems}

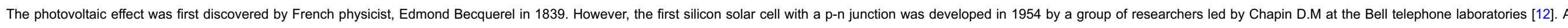
timeline of the progress on photovoltaic solar energy conversion is shown in Table 1 [13]. Photovoltaic systems can be grouped into three generations and they are [14]:

1) First generation systems: These are fully commercial systems based on crystalline silicon technology. They include monocrystalline and polycrystalline silicon cells.

2) Second generation systems: These are based on the photovoltaic thin film and they include, amorphous silicon, cadmium telluride and indium copper selenide, indium and gallium-diselenide.

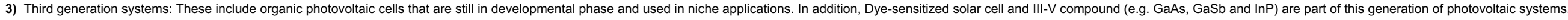

Table 1 Relevant dates to photovoltaic solar energy conversion [13] 
Nevill Mott and Walter Schottky

John Bardeen, Walter Brattain and William Shockley

Daryl Chapin, Calvin Fuller and Gerald Pearson

D.C Reynold, G. Leies, L.L. Antes and R.E. Marburger

Developed the theory of solid-state rectifier (diode)

Solar cells were used for the first time on an orbiting satellite Vanguard 1

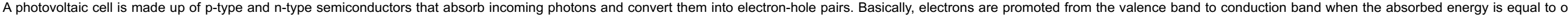

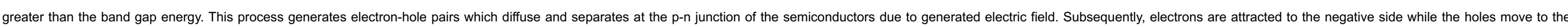

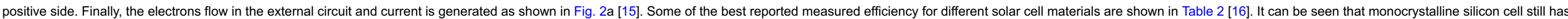
the best conversion efficiency however, PV material optimization research is still on-going and better efficiency values could be achieved in the future.

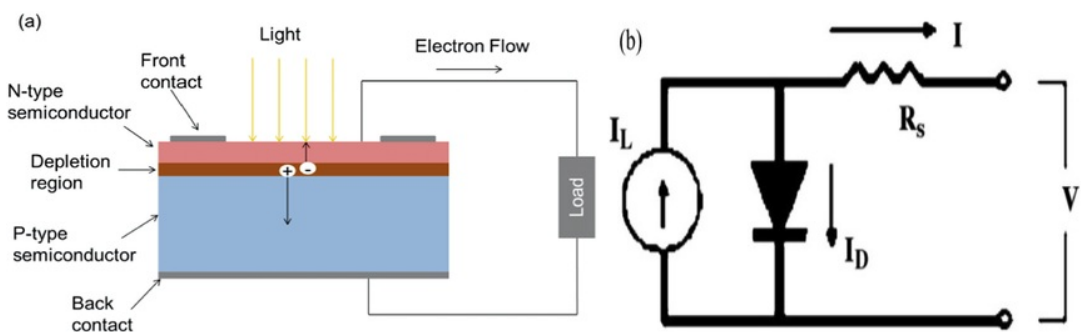

Fig. 2 Photovoltaic cell (a) p-n junction structure [15] and (b) simplified equivalent circuit [13]. alt-ext: Fig. 2

Table 2 Confirmed cell efficiency measured under the global Air Mass (AM) 1.5 , spectrum $\left(1000 \mathrm{~W} / \mathrm{m}^{2}\right)$ at $25^{\circ} \mathrm{C}[16]$. alt-text: Table 2

\begin{tabular}{|c|c|c|c|}
\hline Cell type & Efficiency (\%) & Description & Reference \\
\hline Silicon, (monocrystalline cell) & $26.7 \pm 0.5$ & Kaneka, n-type rear IBC & [133] \\
\hline Silicon, (polycrystalline cell) & $22.3 \pm 0.4^{\prime}$ & FhG-ISE, n-type & [134] \\
\hline III-V, GaAs & $28.8 \pm 0.9$ & Alta devices & [135] \\
\hline III-V, InP & $24.2 \pm 0.5$ & NREL & [136] \\
\hline Thin film, CIGS & $22.9 \pm 0.5$ & Solar frontier & {$[137,138]$} \\
\hline Thin film, CdTe & $21.0 \pm 0.4$ & First solar, on glass & [139] \\
\hline Amorphous silicon & $10.2 \pm 0.3$ & AIST & [140] \\
\hline Perovskite & $20.9 \pm 0.7$ & KRICT & [141] \\
\hline Dye sensitized & $11.9 \pm 0.4$ & Sharp & [142] \\
\hline
\end{tabular}




\subsection{Modelling of PV cell}

A photovoltaic cell can be modelled as a current source with a parallel diode as shown in Fig. 2b. The diode current can be obtained from the Shockley equation as [13]:

$$
I_{D}=\mathrm{I}_{0}\left[\exp \left(\frac{\mathrm{q}\left(\mathrm{V}+\mathrm{I} R_{S}\right)}{\gamma k T_{c}}\right)-1\right]
$$

Reverse saturation current is obtained as,

$$
\mathrm{I}_{0}=\mathrm{DT}_{a b}^{3} \exp \frac{\mathrm{q} \varepsilon_{G}}{A k \mathrm{~T}_{a b}}
$$

where $D$ is the diode diffusion factor, $T_{a b}$ is absolute temperature, $q$ is electron charge, $\varepsilon_{G}$ is material band gap energy, $k$ is Boltzmann constant and $A$ is cross sectional area.

Depending on required voltage and current levels, solar cells are connected in series and parallel respectively. The solar cell generator voltage and current can be obtained as,

$$
V_{g}=I_{g} R_{s} \frac{N_{s}}{N_{p}} \ln \left(1+\frac{N_{p} \mathrm{I}_{p h}-I_{g}}{N_{p} \mathrm{I}_{0}}\right)
$$

where $R_{s}$ is the series resistance, $N_{s}$ is number of cells in series, $N_{p}$ is number of cells in parallel and $I_{p h}$ is the cell photocurrent proportional to solar irradiance.

$$
I_{g}=\mathrm{I}_{p h}-\mathrm{I}_{0} \exp \left(\frac{\mathrm{q} V_{g}}{k T}-1\right)
$$

where $T$ is the cell temperature.

The PV cell short circuit current $\left(I_{s c}\right)$ can be obtained by setting $V_{g}=0$ and $I_{s c}=I_{p h}$. This value varies with cell irradiance and the PV cell open circuit voltage $\left(V_{o c}\right)$ can be obtained by setting $I_{g}=0$ thus,

$$
\mathrm{V}_{o c}=\frac{k T}{\mathrm{q}} \ln \left[\frac{\mathrm{I}_{p h}}{\mathrm{I}_{0}}\right]
$$

The maximum output power of the PV is expressed as,

$$
\frac{\mathrm{d}\left(V_{g} \times I_{g}\right)}{d t}=0
$$

$\mathrm{V}_{m p}=\mathrm{V}_{o c}-\frac{k T}{\mathrm{q}} \ln \left[\frac{\mathrm{V}_{m p}}{k t / \mathrm{q}}+1\right]$

The fill factor (FF) can be expressed as,

$$
F F=\frac{\mathrm{V}_{m p} \times \mathrm{I}_{m p}}{\mathrm{~V}_{o c} \times \mathrm{I}_{s c}}
$$

The efficiency of the PV can be expressed as,

$$
\eta_{p v}=\frac{F F \times \mathrm{V}_{o c} \times \mathrm{I}_{s c}}{\mathrm{P}_{i n}}
$$

where $P_{\text {in }}$ is the incident power on the PV cell.

\subsection{Influence of temperature on photovoltaic cells}

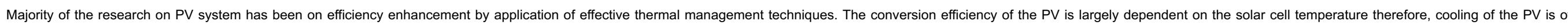

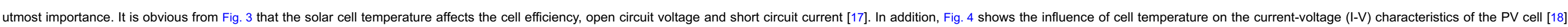




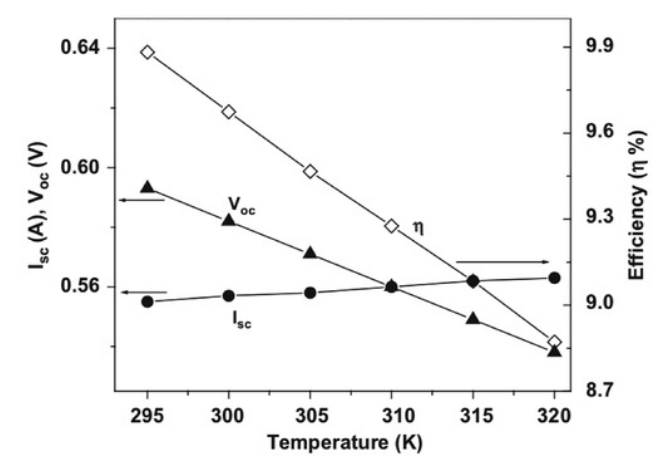

Fig. 3 Effect of cell temperature on efficiency, open circuit voltage and short circuit current of a monocrystalline silicon cell [17]

alt-text: Fig. 3

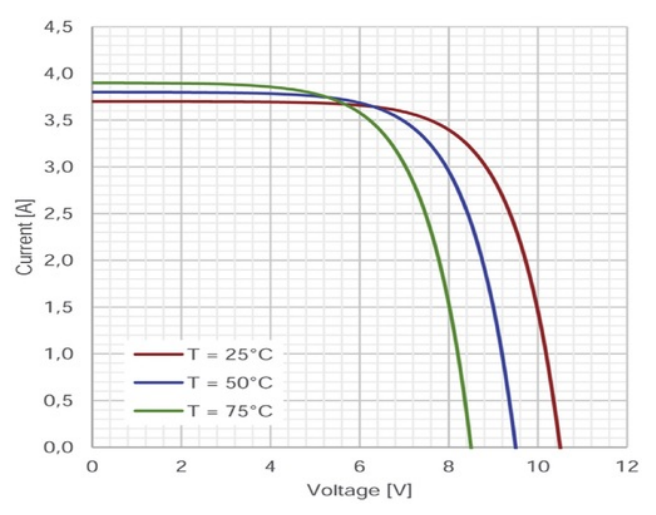

Fig. 4 Influence of temperature on the I-V characteristics of a photovoltaic cell [18] alt-text: Fig. 4

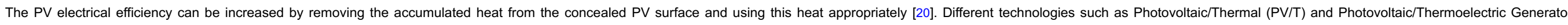

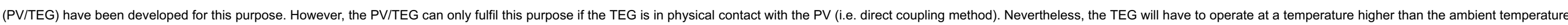
to produce some electrical power and it most likely will heat up the solar cell if not properly cooled. If the TEG is not in physical contact with the PV (i.e. spectrum splitting method), it cannot cool down the PV cell.

\section{Thermoelectric devices}

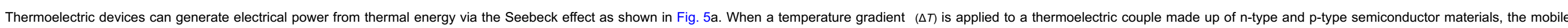

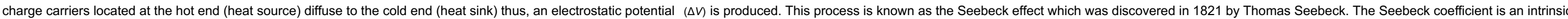
thermoelectric material property which is expressed as, 


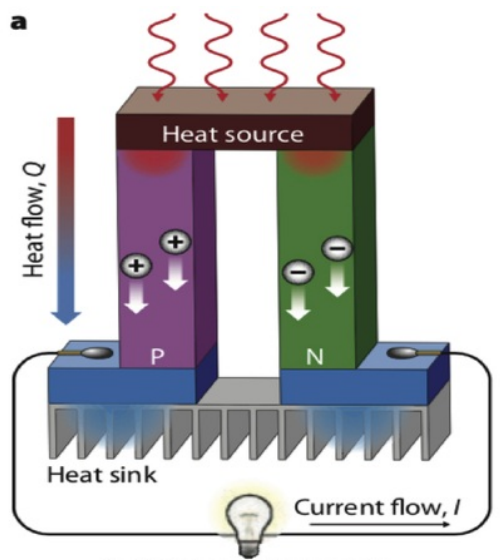

Power generation mode

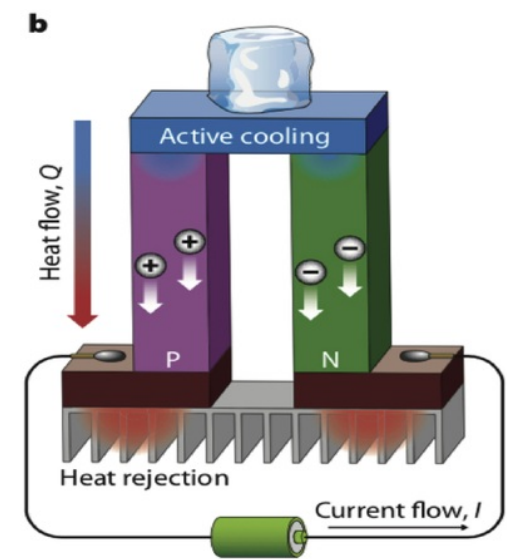

Active refrigeration mode

Fig. 5 Schematic of a thermoelectric (a) generator and (b) cooler [21]

alt-text: Fig. 5

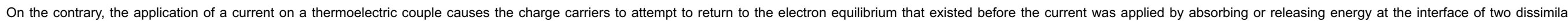
materials. This process is known as the Peltier effect which was discovered in 1834 by Jean-Charles Peltier and it is shown in Fig. 5b [21].

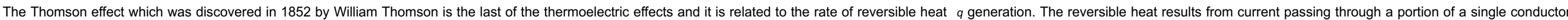
along which there is a temperature difference. Although Thomson effect is not of primary importance in thermoelectric devices, it is still essential for detailed calculations as it influences the device performance [22]

\subsection{Thermoelectric materials}

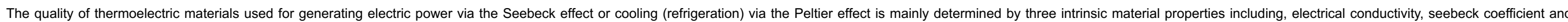

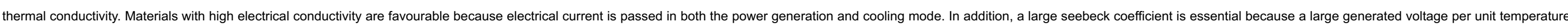

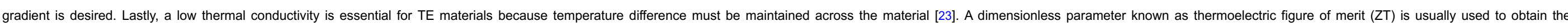
thermoelectric efficiency and it is expressed as [24],

$$
Z T=\frac{\alpha^{2} \sigma}{\kappa} \mathrm{T}
$$

where $\alpha$ is the Seebeck coefficient, $\sigma$ is the electrical conductivity, $k$ is the thermal conductivity and $T$ is the absolute temperature.

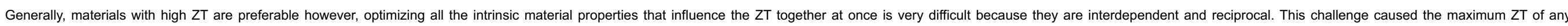

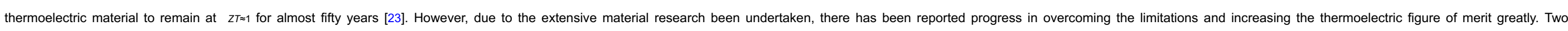

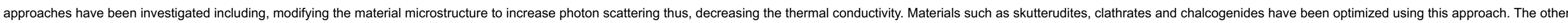

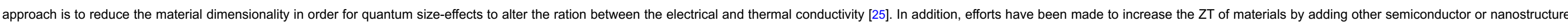

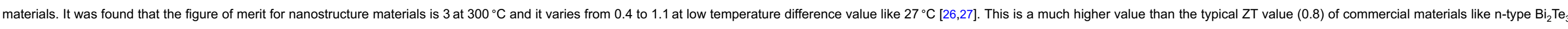
and p-type $\mathrm{Sb}_{2} \mathrm{Te}_{3}$ at temperatures below $150^{\circ} \mathrm{C}$ [24]. Super-lattice structure, plasma treatment and material segmentation are the other methods used in developing thermoelectric materials with high efficiency [28].

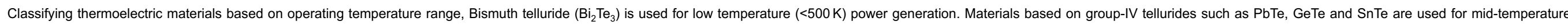

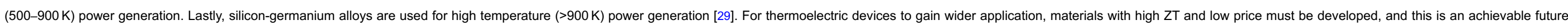
goal due to the extensive research being carried out in this area. 


\subsection{Modelling of thermoelectric generator and cooler}

The efficiency of a thermoelectric generator is expressed as [22]

Energy supplied to the load

$\eta=\overline{\text { Heat energy abosrbed at the hot junction }}$

Assuming constant thermoelectric material properties and negligible contact resistances, the efficiency can be expressed as,

$\eta_{\text {teg }}=\frac{\mathrm{I}^{2} \mathrm{R}}{\alpha I T_{H}=\kappa\left(T_{H}-T_{C}\right)-\frac{1}{2} \mathrm{I}^{2} \mathrm{R}}$

where $I$ is current, $R$ is series resistance, $T_{H}$ is hot side temperature and $T_{C}$ is cold side temperature.

The maximum conversion efficiency is given as,

$\eta_{\max }=\eta_{c} \frac{\sqrt{1+Z T}-1}{\sqrt{1+Z T}+\frac{T_{C}}{T_{H}}}$

where $\eta_{c}$ is the Carnot efficiency and it is expressed as,

$\eta_{c}=\frac{T_{H}-T_{C}}{T_{H}}$

The energy efficiency of the thermoelectric cooler is measured in terms of its coefficient of performance (COP) and it is expressed as [22]

$C O P=\frac{\text { Heat absorbed }}{\text { Electrical power input }}=\frac{\alpha I T_{C}-\frac{1}{2} \mathrm{I}^{2} \mathrm{R}-\kappa\left(T_{H}-T_{C}\right)}{\alpha I\left(T_{H}-T_{C}\right)+\mathrm{I}^{2} \mathrm{R}}$

The current $\overline{\mathrm{I}}$ for maximum cooling power is expressed as,

$\overline{\mathrm{I}}=\frac{\alpha T_{C}}{\mathrm{R}}$

The maximum coefficient of performance is given as,

$C O \mathrm{P}_{\max }=\frac{T_{C}\left[(1+Z T)^{\frac{1}{2}}-\frac{T_{H}}{T_{C}}\right]}{\left(T_{H}-T_{C}\right)\left[(1+Z T)^{\frac{1}{2}}+1\right]}$

As in the case of the thermoelectric generator, the figure of merit (ZT) also determines the maximum coefficient of performance that can be achieved.

\subsection{Applications of thermoelectric generator and cooler}

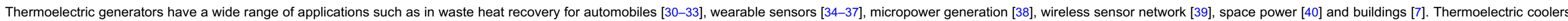

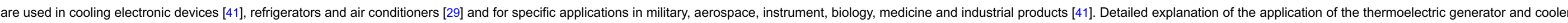
in the aforementioned sectors can be found in the referenced literatures. For the sake of this review, more focus is placed on the application of hybrid PV/TEG in the later sections.

\section{Hybrid photovoltaic/thermoelectric generator (PV/TEG)}

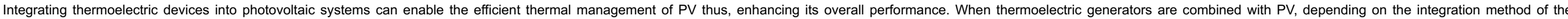

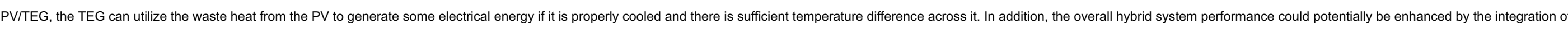
thermoelectric generators into PV if the system is properly designed although the is a possibility of reduced performance due to the complex relationship between PV and TEG. 


\subsection{Modelling of hybrid PV/TEG}

Generally, the overall efficiency of the hybrid PV/TEG is a sum of the individual efficiencies of the PV $\left(\eta_{p v}\right)$ and TEG $\left(\eta_{\text {teg }}\right)$ and it can be expressed as [11]:

$\eta_{p v / t e g}=\eta_{p v}+\eta_{t e g}=\frac{\mathrm{P}_{p v}}{\mathrm{P}_{i n} \mathrm{~A}_{p v}}+\frac{\mathrm{P}_{t e g}}{\mathrm{P}_{i n} \mathrm{~A}_{p v}}$

where $P_{p v}$ is the power output of the solar cell, $P_{\text {teg }}$ is the TEG power output, $A_{p v}$ is the solar cell area and $P_{i n}$ is the input solar power.

The above Eq. (19) is applicable for a simplified design of a hybrid PV/TEG where the PV and TEG are thermally coupled but electrically separated.

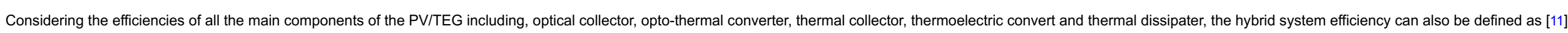
$\eta_{p v / t e g}=\eta_{p v}+\eta_{\text {teg }}=\eta_{p v}+\eta_{\text {opt }} \eta_{o t} \eta_{\text {teg }} \eta_{\text {diss }}$

where $\eta_{o p t}$ is the optical collector efficiency, $\eta_{o t}$ is the opto-thermal efficiency and $\eta_{\text {diss }}$ is the thermal dissipater efficiency

The optical collector efficiency is given as:

$\eta_{\text {opt }}=\frac{\mathrm{CP}_{\text {in }} \mathrm{A}_{\text {opt }} \tau_{\text {opt }}}{\mathrm{CP}_{\text {in }} \mathrm{A}_{\text {opt }}}=\tau_{\text {opt }}$

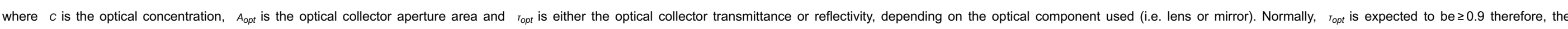
optical collector can be assumed to not absorb power thus, it does not heat up. Consequently, $\eta_{o p t}$ can be considered as temperature independent.

The thermal dissipater efficiency is given as:

$\eta_{\text {diss }}=1-\frac{\mathrm{P}_{\text {diss }}}{\mathrm{P}_{\text {steg }}^{\text {out }}}$

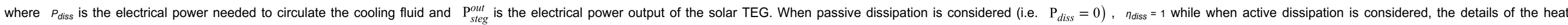
dissipater geometry has to be considered.

The opto-thermal efficiency of the hybrid system is given as:

$\eta_{o t}=\alpha_{o t c o n v} \tau_{\text {enc }} \tau_{h m}\left[1-\left(\eta_{p v}+\mathrm{R}_{p v}+\mathrm{T}_{p v}\right)\right]-\frac{\sigma \mathrm{A}_{a b s}\left[\varepsilon_{p v}^{\prime}\left(T_{h}^{4}-T_{a}^{4}\right)+\varepsilon_{\text {thcol }}\left(T_{h}^{4}-T_{c}^{4}\right)\right]}{\mathrm{CP}_{\text {in }} \tau_{\text {opt }} \mathrm{A}_{\text {opt }}}$

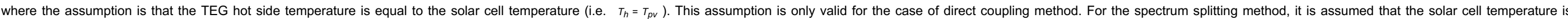

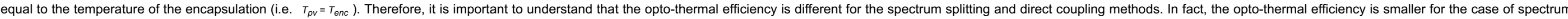

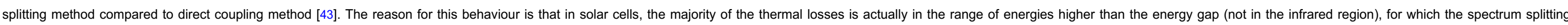

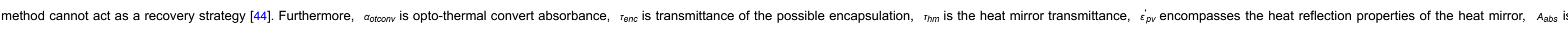
opto-thermal converter area, $T_{a}$ is ambient temperature and $R_{p v}$ is the reflected fraction of solar power by the solar cell.

The resultant emittance of the TEG parallel surfaces can be defined as [45]:

$$
\varepsilon_{\text {thcol' }}=\frac{1}{\frac{1}{\varepsilon_{\text {thcol }}}+\frac{1}{\varepsilon_{c}}-1}
$$

where $\varepsilon_{\text {thcol }}$ is the thermal collector emittance and $\varepsilon_{c}$ is emittance. 


$$
\varepsilon_{p v}^{\prime}=\varepsilon_{p v}\left(1-\eta_{h m}^{\mathrm{r}}\right)
$$

where $\eta_{h m}^{\mathrm{r}}$ is the back-reflecting efficiency for the heat coming from the solar cell and it is defined as:

$\eta_{h m}^{\mathrm{r}}=\left[\left(\frac{\int \mathrm{R}_{h m}(\lambda) d \lambda}{\int d \lambda}\right)_{i r}\right]$

where $R_{h m}(\lambda)$ is the reflectance of the heat mirror and the subscript 'ir' represents the evaluation of the integral over a range of wavelengths from 2500 to $30,000 \mathrm{~nm}$.

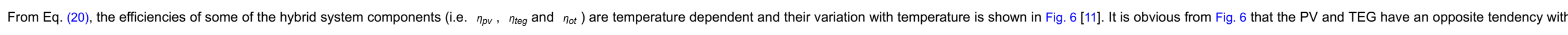

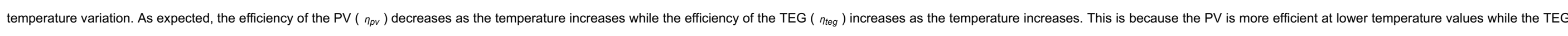

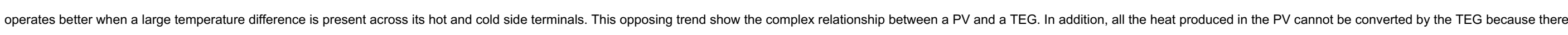

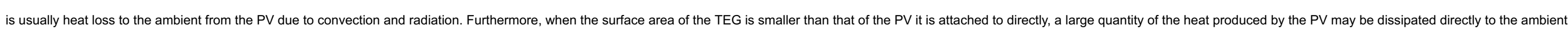
without passing through the TEG.

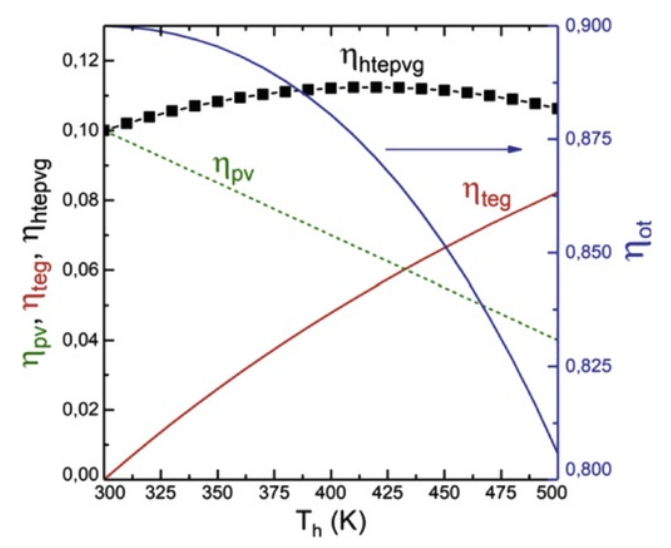

Fig. 6 Variation of hybrid system and individual components efficiency with temperature [11]

alt-text: Fig. 6

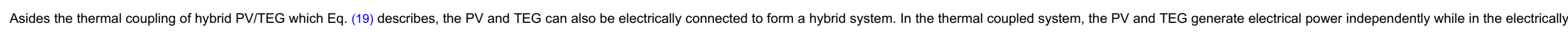

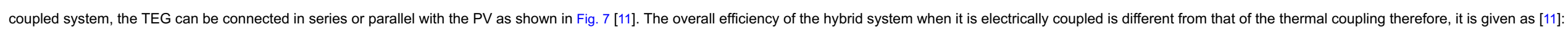

$\eta_{p v / t e g}=\eta_{p v}+\eta_{s t e g}-\left(1-\eta_{e l}\right)=\frac{\mathrm{P}_{p v}+\mathrm{P}_{s t e g}-\mathrm{P}_{e l-l o s s}}{\mathrm{P}_{i n} \mathrm{~A}_{p v}}$

where $\eta_{\text {steg }}$ is the solar TEG efficiency, $\eta_{e l}$ is the electrical hybridization efficiency and $P_{e l-l o s s}$ is the electrical power loss. 


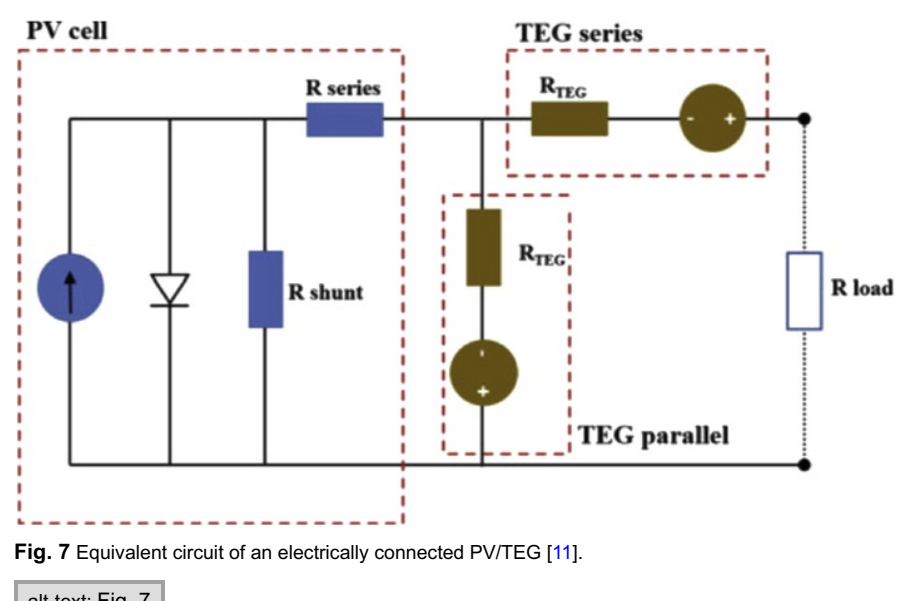

alt-text: Fig. 7

\subsection{Hybrid system integration methods}

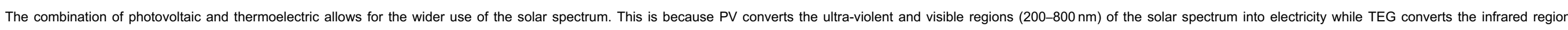

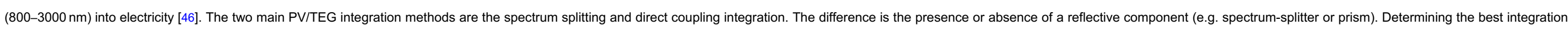

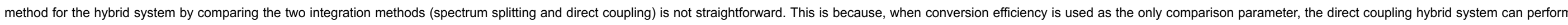

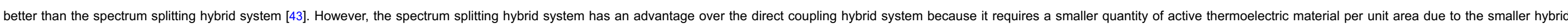

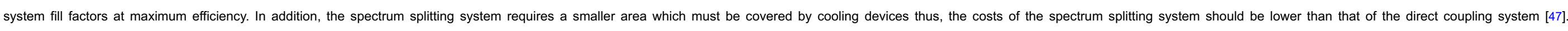

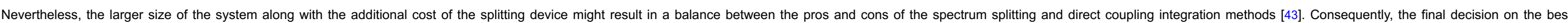

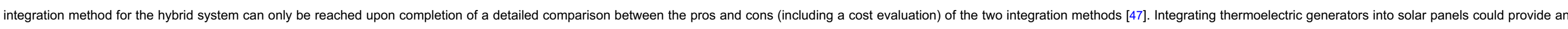

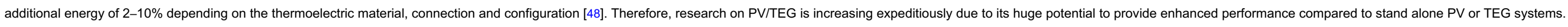

\subsubsection{Spectrum splitting method}

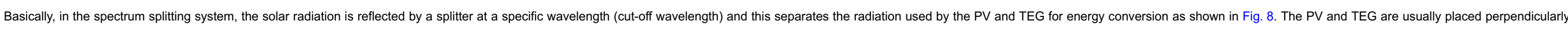

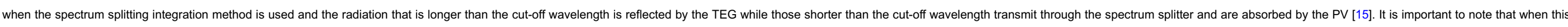
integration method is used, the PV and TEG work independently on converting solar energy into electricity thus, the TEG doesn't cool down the PV or use the PV's waste heat for energy conversion.

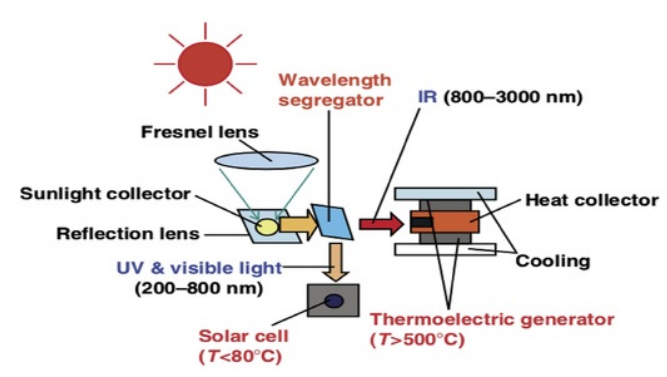

Fig. 8 Schematic of spectrum splitting PV/TEG integration [46]

alt-text: Fig. 8

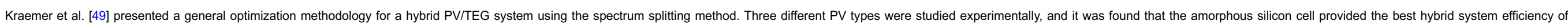




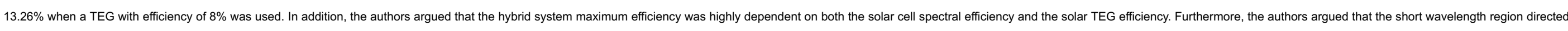
to the solar TEG is usually only a small portion of the total solar spectrum thus, it could be neglected. Regardless, it is worth noting that the authors used an assumed efficiency value for the TEG which may not be practical.

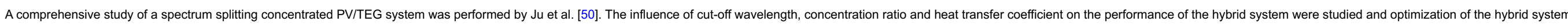

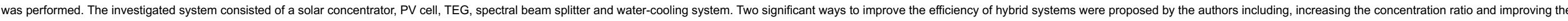

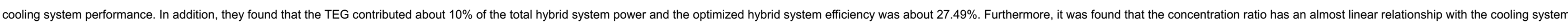

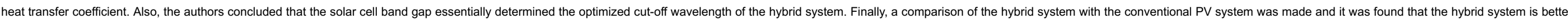
suited for high concentration conditions due to its enhanced performance. Although the results from this research are significant, an experimental validation of the simulation results wasn't performed.

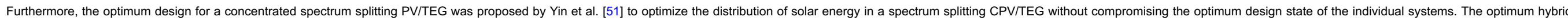

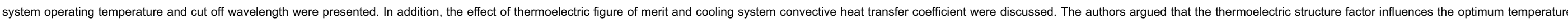

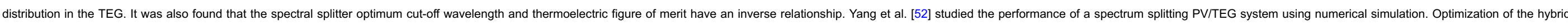

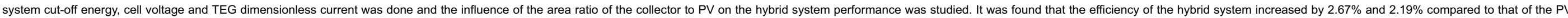
only system at concentration factors of 30 and 100 respectively.

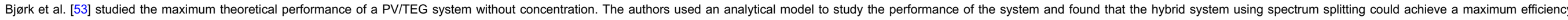

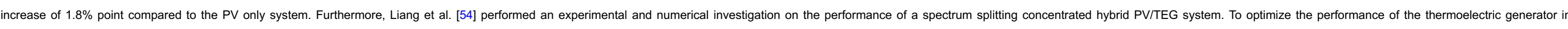

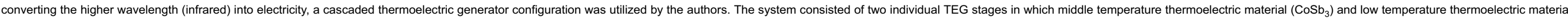

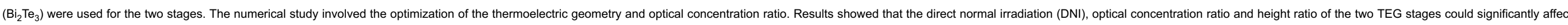

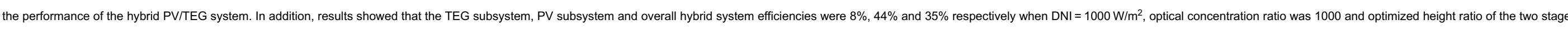
TEG was 0.6. The performance data for some of the spectrum splitting PV/TEG systems reviewed can be found in Table 4

Table 3 Power output and efficiency enhancement of hybrid system using different cooling systems [103] alt-text: Table 3

\begin{tabular}{|c|c|c|c|c|c|}
\hline & Natural cooling & Forced cooling & Water cooling & $\mathrm{SiO}_{2} /$ water nanofluid cooling & $\mathrm{Fe}_{3} \mathrm{O}_{4} /$ water nanofluid cooling \\
\hline Total power increase (\%) & Base & 4.885 & 5.776 & 8.26 & 6.284 \\
\hline Total efficiency increase (\%) & Base & 1.865 & 3.051 & 3.355 & 3.131 \\
\hline
\end{tabular}

Table 4 Summary of some selected spectrum splitting PV/TEG systems reviewed.

alt-text: Table 4

Reference silicon

Amorphous silicon

Polymer thin film

Ju et al. [50]

GaAs

Mizoshiri et al. [144] Amorphous silicon
Materia

N/A

N/A

N/A

Skutterudite

$\mathrm{CoSb}_{3}$

Thin- film Bismuth Experiment
Study type

Simulation

Simulation

Simulation

Simulation

Efficiency

PV/TEG

$11.45 \%$

$13.26 \%$

$8.32 \%$

$27.49 \%$

N/A
Remarks

PV

$9.09 \%$ TEG efficiency of $8 \%$ corresponding to figure of merit $(Z T=1.7)$ was used

$9.40 \%$

$3.41 \%$

N/A Figure of merit $(Z T=1.4)$ at $800 \mathrm{~K}$, heat transfer coefficient of $4500 \mathrm{~W} / \mathrm{m}^{2} / \mathrm{K}$ were used, and the optimized results were given.

Open circuit voltage of hybrid system increased by $1.3 \%$ compared to PV only system. 


\begin{tabular}{|c|c|c|c|c|c|c|}
\hline Li et al. [95] & N/A & N/A & Simulation & $31-34 \%$ & N/A & Figure of merit $(\mathrm{ZT}=1)$ was used and $30 \%$ power output enhancement was obtained. \\
\hline Elsarrag et al. [145] & $\begin{array}{l}\text { Monocrystalline } \\
\text { silicon }\end{array}$ & Bismuth telluride & $\begin{array}{l}\text { Experiment and } \\
\text { simulation }\end{array}$ & N/A & N/A & Hybrid system performed better than PV only system. \\
\hline \multirow{2}{*}{$\begin{array}{l}\text { Skjølstrup et al. } \\
\text { [146] }\end{array}$} & Amorphous silicon & N/A & Simulation & $19.1 \%$ & $15.8 \%$ & Beam splitter layer was 114 and TE efficiency was $8 \%$. \\
\hline & $\begin{array}{l}\text { Microcrystalline } \\
\text { silicon }\end{array}$ & N/A & Simulation & $19.8 \%$ & $17.5 \%$ & Beam splitter layer was 128 and TE efficiency was $8 \%$. \\
\hline Sibin et al. [147] & N/A & N/A & Experiment & N/A & N/A & ITO/Ag/ITO spectral beam splitter coating was developed, and it had a high visible transmittance of $88 \%$. \\
\hline Yin et al. [51] & GaAs & N/A & Simulation & $30 \%$ & N/A & Figure of merit was 1 and cut-off wavelength was equal to maximum wavelength of PV. \\
\hline Yang et al. [52] & Silicon & N/A & Simulation & $40.2 \%$ & $39.32 \%$ & Concentration factor was 100. \\
\hline Bjørk et al. [53] & N/A & N/A & Simulation & $\begin{array}{l}1.8 \% \text { points } \\
\text { increase }\end{array}$ & N/A & Maximum hybrid system efficiency without concentration was studied. \\
\hline Djafar et al. [148] & N/A & Bismuth telluride & Experiment & $\mathrm{N} / \mathrm{A}$ & N/A & Long wavelengths of around $800 \mathrm{~nm}$ were emitted by the halogen lamps for the TEG. \\
\hline Shou et al. [149] & Crystalline silicon & N/A & Simulation & $3.24 \%$ increase & N/A & Hybrid system had a filter at 150 suns. \\
\hline
\end{tabular}

\subsubsection{Direct coupling method}

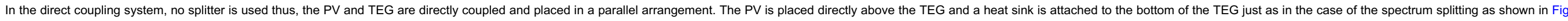

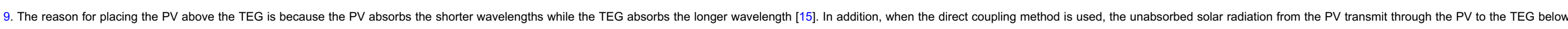
and this serves as the input heat flux for the TEG to generate some electrical power.

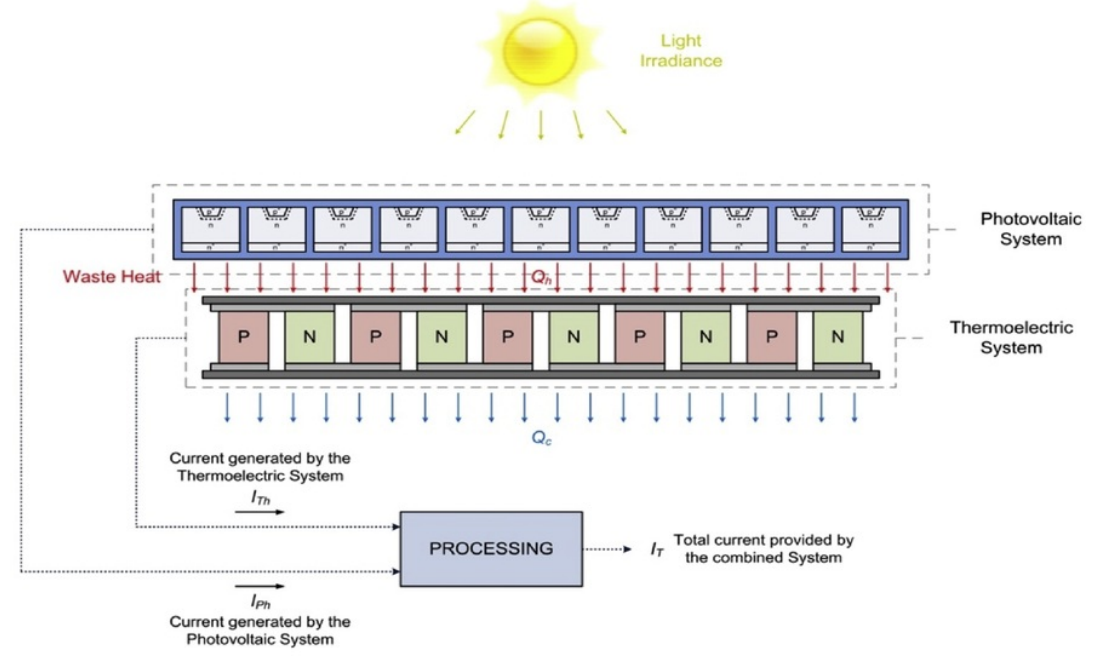

Fig. 9 Schematic of direct coupling PV/TEG integration [18].

alt-text: Fig. 9

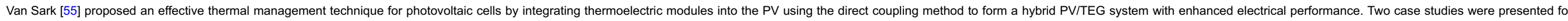

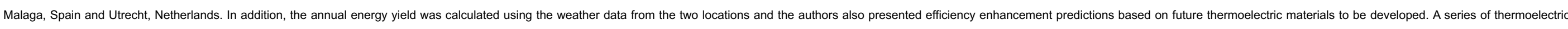

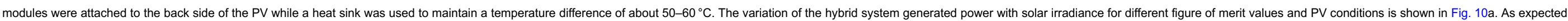




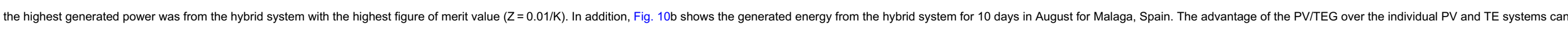

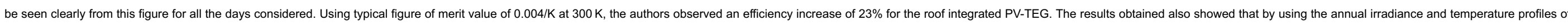

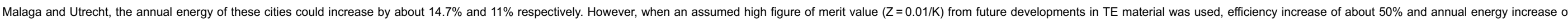
$24.9 \%$ were predicted by the authors. Notwithstanding, this research ignored radiation loss on the front cover and the idealized model developed, overestimated the results by about $10 \%$ for practical PV/TEG systems.
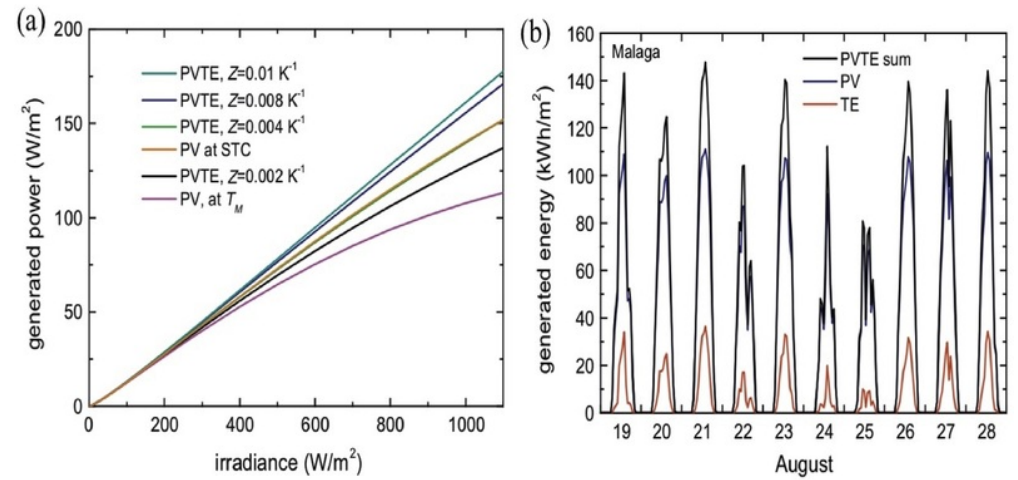

Fig. 10 Hybrid PV/TEG system (a) generated power for four figure of merit Z values and (b) total energy for 10-day period in August for Malaga, Spain [55].

\section{alt-text: Fig. 10}

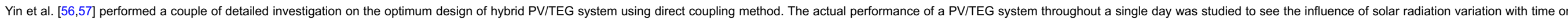

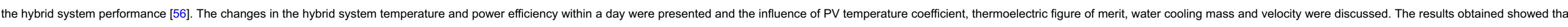

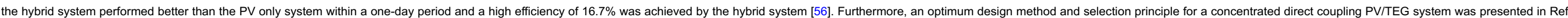

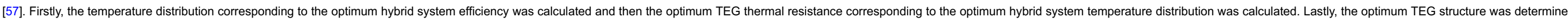

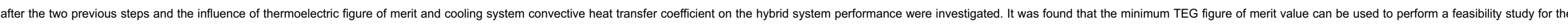

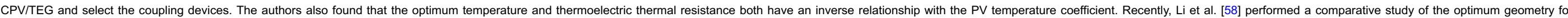

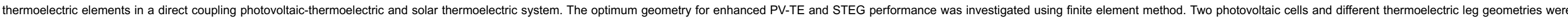

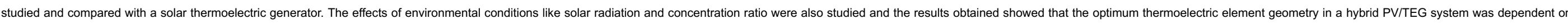
the characteristics of the solar cell used and this optimum geometry is different from that of the solar thermoelectric generator under similar conditions. The performance data for some of the directly coupling PV/TEG systems reviewed can be found in Table 5 .

Table 5 Summary of some selected direct coupling PV/TEG systems reviewed.

\begin{tabular}{|c|c|c|c|c|c|c|}
\hline \multirow[t]{2}{*}{ Reference } & \multicolumn{2}{|r|}{ Material } & \multirow[t]{2}{*}{ Study type } & \multicolumn{2}{|c|}{ Efficiency } & \multirow[t]{2}{*}{ Remarks } \\
\hline & PV & TE & & PV/TEG & PV & \\
\hline Guo et al. [104] & $\begin{array}{l}\text { Dye-sensitized solar cell } \\
\text { (DSSC) }\end{array}$ & N/A & Experiment & $10 \%$ increase & N/A & Hybrid efficiency was compared with a single DSSC. \\
\hline Wang et al. [105] & Dye-sensitized solar cell & N/A & Experiment & $13.8 \%$ & $9.26 \%$ & Solar selective absorber was used. \\
\hline Sark [55] & Polycrystalline silicon & Bismuth telluride $\left(\mathrm{Bi}_{2} \mathrm{Te}_{3}\right)$ & Simulation & $13.98 \%$ & $10.78 \%$ & Typical figure of merit value of 1.2 and coefficient $c=0.058$ were used. \\
\hline Daud et al. [150] & Polycrystalline silicon & Bismuth telluride & Experiment & $9.064 \%$ & $5.970 \%$ & Solar radiation of $868 \mathrm{~W} / \mathrm{m}^{2}$ and liquid cooling was used. \\
\hline Park et al. [79] & Crystalline silicon & Bismuth telluride & $\begin{array}{l}\text { Experiment and } \\
\text { simulation }\end{array}$ & $16.30 \%$ & $12.5 \%$ & $30 \%$ optimized efficiency increase at $15^{\circ} \mathrm{C} \mathrm{TE}$ temperature difference. \\
\hline
\end{tabular}




\begin{tabular}{|c|c|c|c|c|c|c|}
\hline Zhang et al. [151] & Polymer & Bismuth telluride & Experiment & N/A & N/A & Hybrid system power output increase of $46.6 \%$ compared to PV only system was observed. \\
\hline \multirow[t]{2}{*}{ Li et al. [81] } & Crystalline silicon & N/A & Simulation & $11.07 \%$ & $9.5 \%$ & TE load resistance was $0.75 \Omega$ and figure of merit was $0.0085 / \mathrm{K}$. \\
\hline & GaAs & N/A & Simulation & $22.94 \%$ & $21.91 \%$ & TE load resistance was $1.60 \Omega$ and figure of merit was $0.0022 / \mathrm{K}$ \\
\hline \multirow[t]{4}{*}{ Zhang et al. [152] } & Crystalline silicon & \multirow{4}{*}{$\begin{array}{l}\text { Nanostructured bismuth-antimony- } \\
\text { telluride }\end{array}$} & Simulation & $18.6 \%$ & $18.4 \%$ & Concentration ratio was 16. \\
\hline & Thin-film silicon & & Simulation & $14 \%$ & $11 \%$ & Concentration ratio was 12 . \\
\hline & Polymer & & Simulation & $12 \%$ & $4 \%$ & Concentration ratio was 5 . \\
\hline & CIGS & & Simulation & $23.5 \%$ & $21.5 \%$ & Concentration ratio was 30 . \\
\hline \multirow[t]{4}{*}{ Cui et al. [96] } & Crystalline silicon & Bismuth telluride & Simulation & $20.1 \%$ & N/A & Operating temperature was $300 \mathrm{~K}$, optical concentration was 100 and PCM was used. \\
\hline & CIGS & Bismuth telluride & Simulation & $20.5 \%$ & N/A & Operating temperature was $300 \mathrm{~K}$ and optical concentration was 0 . \\
\hline & Single-junction GaAs & Bismuth telluride & Simulation & $28.09 \%$ & N/A & Operating temperature was $425 \mathrm{~K}$, figure of merit was 1.5 , and optical concentration was 500 . \\
\hline & GalnP/InGaAs/Ge (III-V) & Bismuth telluride & Simulation & $38.90 \%$ & N/A & Operating temperature was $300 \mathrm{~K}$ and optical concentration was 500 . \\
\hline Liao et al. [153] & Polycrystalline & Bismuth telluride & Simulation & $15 \%$ & N/A & CG (Concentration ratio x Solar irradiance) was $875 \mathrm{~W} / \mathrm{m}^{2}$. \\
\hline Chen et al. [154] & DSSC & N/A & Simulation & $24.60 \%$ & N/A & Maximum power output of $1.389 \mathrm{~mW}$ was obtained. \\
\hline Lin et al. [82] & Crystalline silicon & Bismuth telluride & Simulation & $13 \%$ & $10.24 \%$ & Power and efficiency enhancement of about $27 \%$ was observed. \\
\hline Beeri et al. [155] & Multijunction & Bismuth telluride & $\begin{array}{l}\text { Experiment and } \\
\text { simulation }\end{array}$ & $32.09 \%$ & $32.08 \%$ & Concentration factor was 20 and hybrid power output was $0.190 \mathrm{~W}$. \\
\hline Da et al. [123] & GaAs & N/A & Simulation & $18.51 \%$ & N/A & Figure of merit was 2.5 and Air Mass was 1.5. \\
\hline Dou et al. [106] & DSSC & $\mathrm{Bi}_{2} \mathrm{Te}_{3} / \mathrm{ZnO}$ & Simulation & $4.27 \%$ & N/A & Hybrid efficiency was $44.3 \%$ higher than efficiency of $\mathrm{ZnO}$ photoanode. \\
\hline $\begin{array}{l}\text { Attivissimo et al. } \\
\text { [156] }\end{array}$ & Polycrystalline & Bismuth telluride & Simulation & N/A & N/A & TEG contributes about $12.2 \%$ to the hybrid system energy in Pachino. \\
\hline Luo et al. [157] & Heterojunction & Bismuth telluride & Experiment & $\begin{array}{l}23.30 \% \\
\text { increase }\end{array}$ & N/A & Efficiency increase was achieved after 1 min illumination. \\
\hline Pang et al. [158] & Monocrystalline silicon & Bismuth telluride & Simulation & $5.9 \%$ & $5.7 \%$ & Efficiency increase of $3.9 \%$ was observed. \\
\hline \multirow[t]{3}{*}{ Cotfas et al. [159] } & Monocrystalline silicon & Bismuth telluride & Simulation & N/A & $18.93 \%$ & Solar irradiance was $920 \mathrm{~W} / \mathrm{m}^{2}$. \\
\hline & Polycrystalline silicon & Bismuth telluride & Simulation & N/A & $16.71 \%$ & Solar irradiance was $1020 \mathrm{~W} / \mathrm{m}^{2}$. \\
\hline & Amorphous silicon & Bismuth telluride & Simulation & N/A & $2.88 \%$ & Solar irradiance was $720 \mathrm{~W} / \mathrm{m}^{2}$. \\
\hline Lamba et al. [75] & Monocrystalline silicon & Bismuth telluride & Simulation & $5.8 \%$ & $5.2 \%$ & Number of TEG was 127 and concentration ratio was 3. \\
\hline Zhu et al. [74] & Monocrystalline silicon & N/A & $\begin{array}{l}\text { Experiment and } \\
\text { simulation }\end{array}$ & $23 \%$ & $19 \%$ & TEG contributed extra electrical energy of $648 \mathrm{~J}$ during zero solar radiation period. \\
\hline Hashim et al. [90] & Amorphous silicon & Bismuth telluride & Simulation & $10.2 \%$ & N/A & Hybrid system power output increased to $163 \mathrm{~mW}$. \\
\hline \multirow[t]{2}{*}{$\begin{array}{l}\text { Kossyvakis et al. } \\
\text { [92] }\end{array}$} & Polycrystalline silicon & Bismuth telluride & $\begin{array}{l}\text { Experiment and } \\
\text { simulation }\end{array}$ & $\begin{array}{l}22.5 \% \\
\text { increase }\end{array}$ & N/A & Hybrid system efficiency was obtained theoretically. \\
\hline & DSSC & Bismuth telluride & $\begin{array}{l}\text { Experiment and } \\
\text { simulation }\end{array}$ & $\begin{array}{l}30.2 \% \\
\text { increase }\end{array}$ & N/A & Hybrid system efficiency was obtained theoretically. \\
\hline Zhang et al. [111] & Perovskite & Bismuth telluride & Simulation & $18.6 \%$ & $17.8 \%$ & Solar selective absorber was used. \\
\hline Cui et al. [97] & Single-junction GaAs & Bismuth telluride & Experiment & $13.45 \%$ & $13.43 \%$ & Phase change material (PCM) was used. \\
\hline Zhou et al. [160] & DSSC & p-type $\mathrm{Bi}_{0.4} \mathrm{Sb}_{1.6} \mathrm{Te}_{3}, \mathrm{n}$-type & Experiment & $9.08 \%$ & $7.21 \%$ & Hybrid efficiency was greater than TEG efficiency by $725.5 \%$. \\
\hline
\end{tabular}




\begin{tabular}{|c|c|c|c|c|c|c|}
\hline & & $\mathrm{Bi}_{2.85} \mathrm{Se}_{0.15} \mathrm{Te}_{3}$ & & & & \\
\hline Lamba et al. [76] & Monocrystalline silicon & Bismuth telluride & Simulation & $7.44 \%$ & $7.068 \%$ & Maximum power output of the hybrid system was $595.5 \mathrm{~mW}$. \\
\hline Dallan et al. [114] & Monocrystalline silicon & Bismuth telluride & Experiment & $13.2 \%$ & $8.052 \%$ & PV and TE power output were $60.5 \mathrm{~W} / \mathrm{m}^{2}$ and $0.01 \mathrm{~W} / \mathrm{m}^{2}$ respectively. \\
\hline Kil et al. [161] & Single junction GaAs & Bismuth telluride & Experiment & $23.2 \%$ & $22.5 \%$ & Solar concentration was 50 suns. \\
\hline Soltani et al. [103] & Crystalline silicon & Bismuth telluride & Experiment & $\begin{array}{l}3.355 \% \\
\text { increase }\end{array}$ & N/A & $\begin{array}{l}\mathrm{SiO}_{2} / \text { water nanofluid cooling was used and power output was increase by } 8.26 \% \text { compared to } \\
\text { natural cooling. }\end{array}$ \\
\hline \multirow[t]{3}{*}{ Li et al. [162] } & CIGS & Bismuth telluride & Simulation & $21.6 \%$ & $20.71 \%$ & Concentration ratio was 200 . \\
\hline & Thin film silicon & Bismuth telluride & Simulation & $13.1 \%$ & $12.89 \%$ & Concentration ratio was 200 . \\
\hline & Polymer & Bismuth telluride & Simulation & $8 \%$ & $7.47 \%$ & Concentration ratio was 180 . \\
\hline \multirow[t]{2}{*}{ Contento et al. [43] } & Amorphous silicon & Nanostructured $\mathrm{Bi}_{2} \mathrm{Te}_{3}$ & Simulation & $\approx 57 \%$ increase & N/A & $\approx 57 \%$ increase and $\approx 42 \%$ for directly and indirectly coupled systems respectively. \\
\hline & Heterojunction CZTS & Nanostructured $\mathrm{Bi}_{2} \mathrm{Te}_{3}$ & Simulation & $\approx 35 \%$ increase & N/A & $\approx 35 \%$ increase and $\approx 24 \%$ for directly and indirectly coupled systems respectively. \\
\hline Liu et al. [163] & Perovskite & Bismuth telluride & Experiment & $22.2 \%$ & $9.88 \%$ & Ice bath was used for TE cooling and Air mass was 1.5. \\
\hline Zhang et al. [164] & Silicon & N/A & Experiment & N/A & N/A & Hybrid system achieved high absorption for wavelengths of $0.3-1.1 \mu \mathrm{m}$. \\
\hline $\begin{array}{l}\text { Machrafi et al. } \\
\text { [165] }\end{array}$ & Monocrystalline silicon & $\mathrm{p}-\mathrm{Sb}_{2} \mathrm{Te}_{3} \mathrm{n}-\mathrm{Bi}_{2} \mathrm{Se}_{3}$ & Simulation & $25 \%$ & N/A & Thermoelectric nanoparticles were used, and optimum cooling velocity was $10 \mathrm{~m} / \mathrm{s}$. \\
\hline $\begin{array}{l}\text { Jeyashree et al. } \\
\text { [166] }\end{array}$ & Polycrystalline silicon & Bismuth telluride & Experiment & $\mathrm{N} / \mathrm{A}$ & N/A & Ice block was used for TEG cooling and hybrid system power output was $10.772 \mathrm{~W}$. \\
\hline $\begin{array}{l}\text { Nishijima et al. } \\
\text { [167] }\end{array}$ & Black silicon & N/A & Simulation & N/A & N/A & Ge_ $\mathrm{Sn}$ layer was added to the solar cell and voltage increase of $7 \%$ was observed. \\
\hline Babu et al. [68] & Polycrystalline & Bismuth telluride & Simulation & $6 \%$ increase & $\mathrm{N} / \mathrm{A}$ & TEG contributed energy of $1-3 \%$ of $P V$ rating. \\
\hline Li et al. [168] & $\begin{array}{l}\text { InGaP/InGaAs/Ge triple- } \\
\text { junction }\end{array}$ & Bismuth telluride & Experiment & $33.53 \%$ & 32.86 & PCM and water cooling were used. Average efficiency was considered. \\
\hline
\end{tabular}

\subsection{PV/TEG study type}

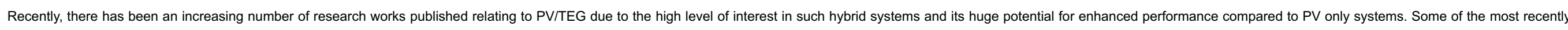

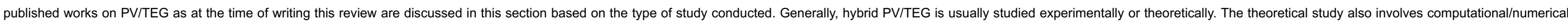
study.

\subsubsection{Experimental study}

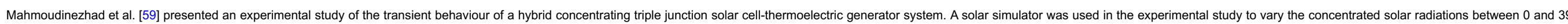

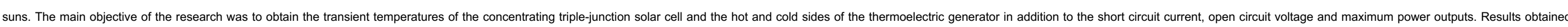

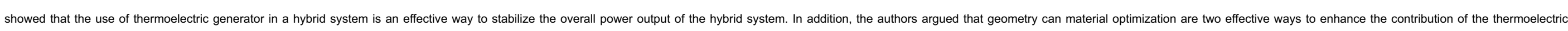
generator to the overall hybrid system power output.

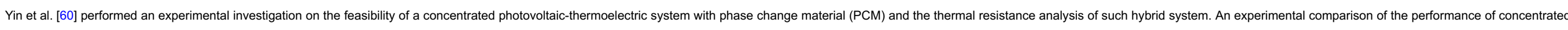

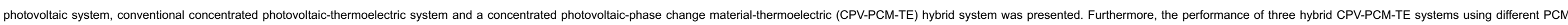

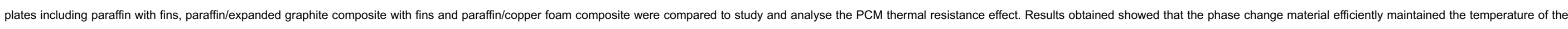

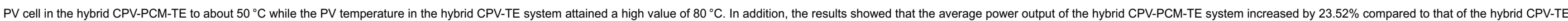




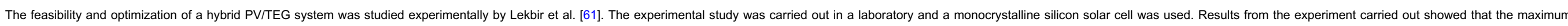

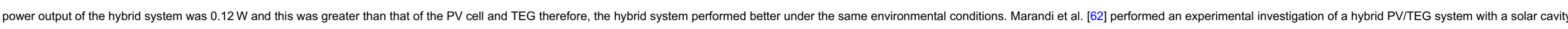

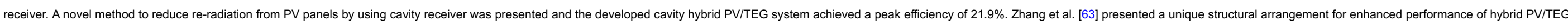

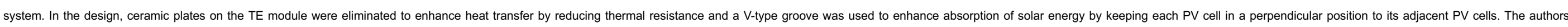

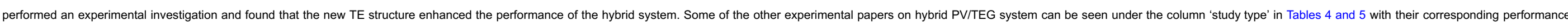
data.

\subsubsection{Theoretical/computational study}

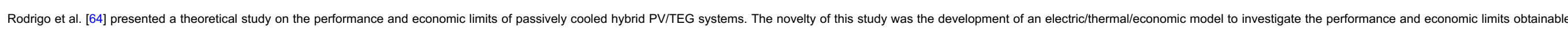

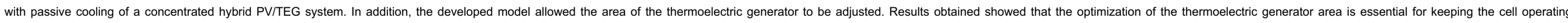

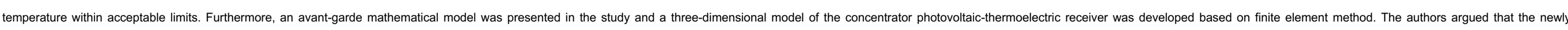
proposed passive cooling concept would influence the development of future passively cooled concentrated PV/TEG prototypes.

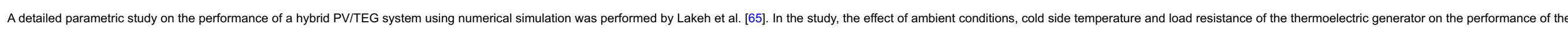

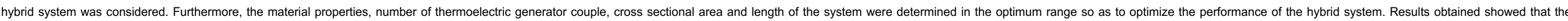

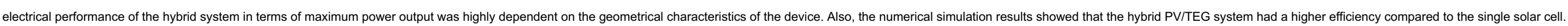

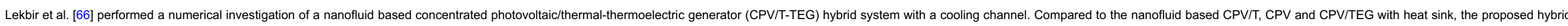

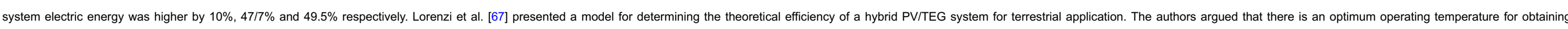

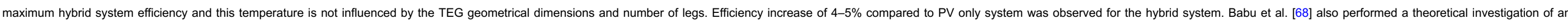
unconcentrated hybrid PV/TEG system using the MATLAB/Simulink environment. It was found that the hybrid system had an overall efficiency increase of $6 \%$ and additional energy projection of $5 \%$.

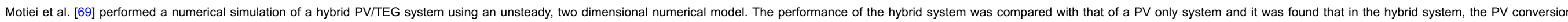

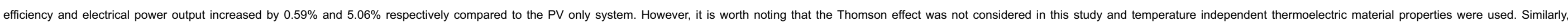

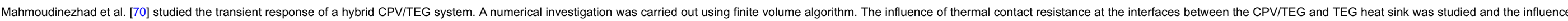

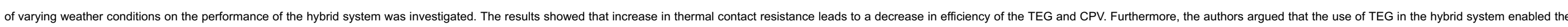
stable generation of power under varying weather condition.

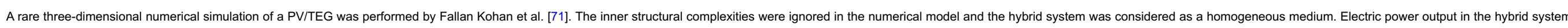

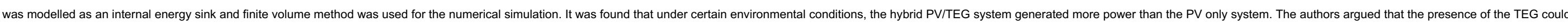

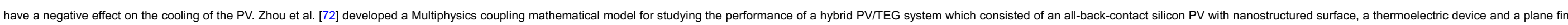

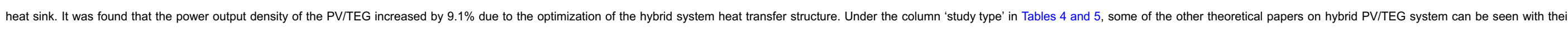
corresponding performance data.

\section{Current research focus areas in hybrid PV/TEG}

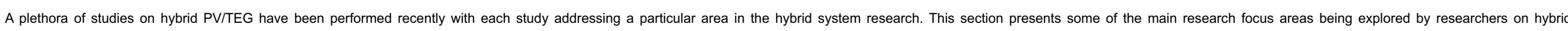
photovoltaic-thermoelectric generator and some niche applications of hybrid PV/TEG.

\subsection{Concentrated hybrid system}

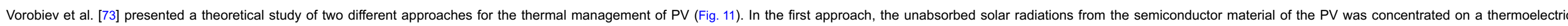




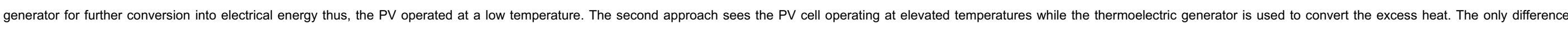

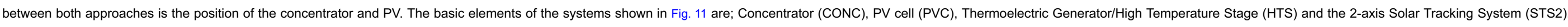

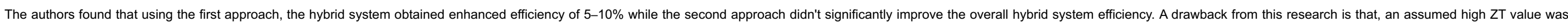
used which is not currently practical.

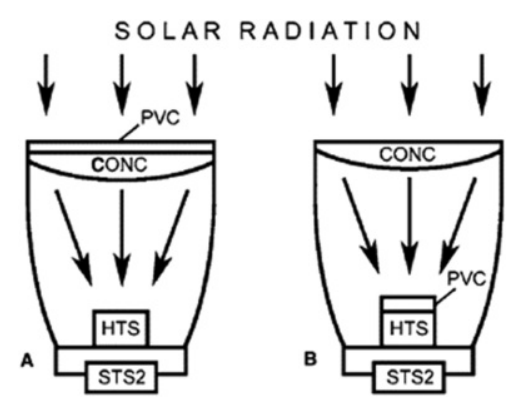

Fig. 11 Schematic diagram of PV/TEG with PV operating at (A) low and (B) high temperatures [73].

\section{alt-text: Fig. 11}

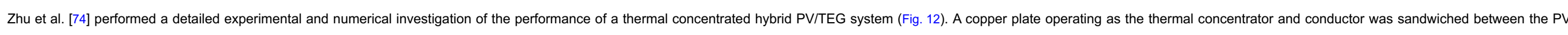

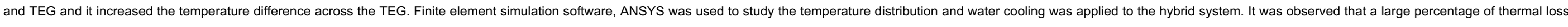

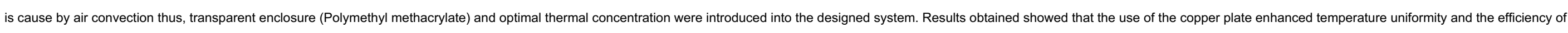
the hybrid system was about $23 \%$.
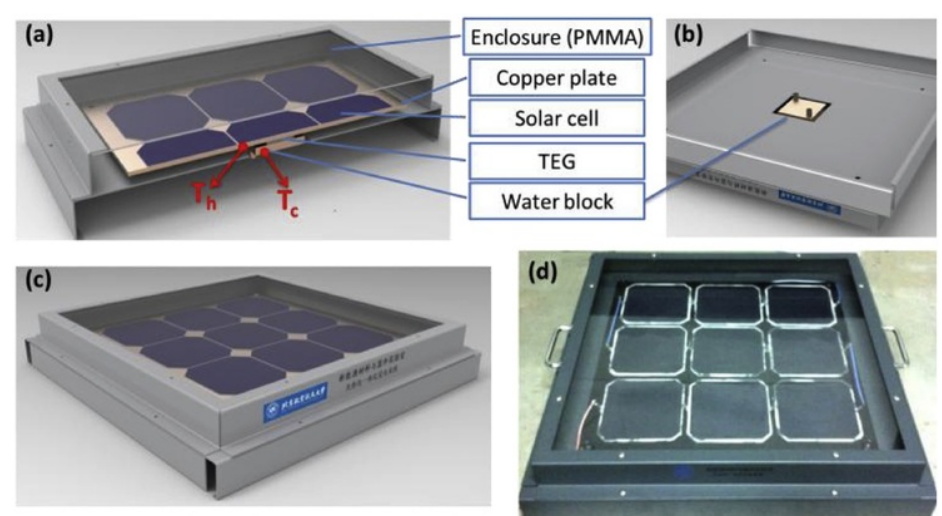

Fig. 12 Hybrid PV/TEG (a) cross-sectional view, (b) bottom view, (c) global view and (d) physical diagram [74] alt-text: Fig. 12

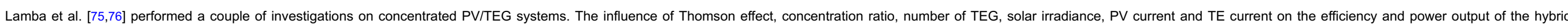

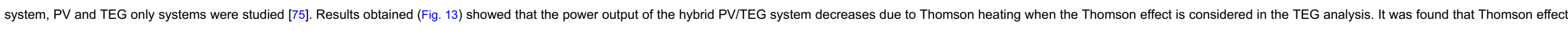

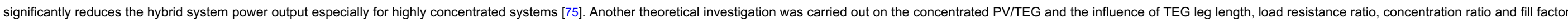

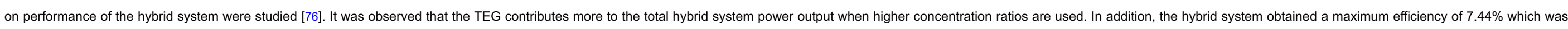
$5 \%$ higher than that of the PV only system [76]. 


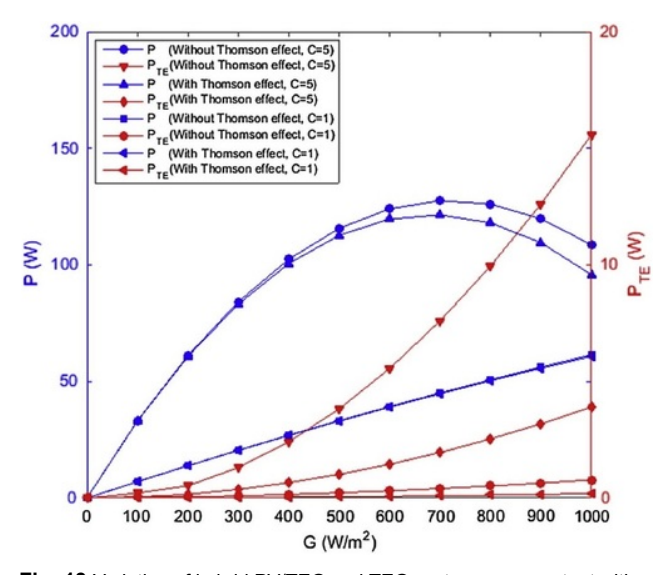

Fig. 13 Variation of hybrid PV/TEG and TEG system power output with solar irradiance [75].

\section{alt-text: Fig. 13}

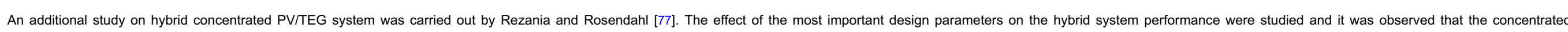

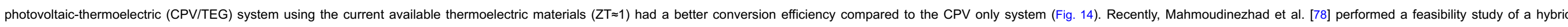

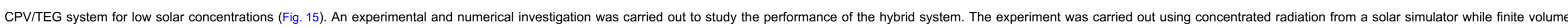

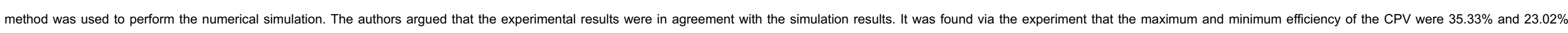
respectively.

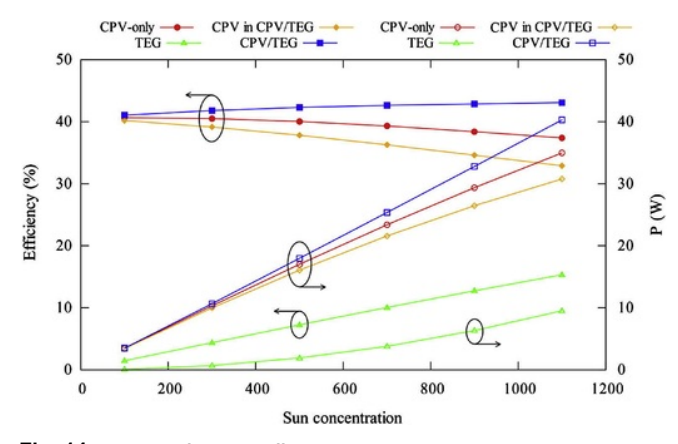

Fig. 14 Variation of system efficiency with sun concentration [77].

alt-text: Fig. 14 

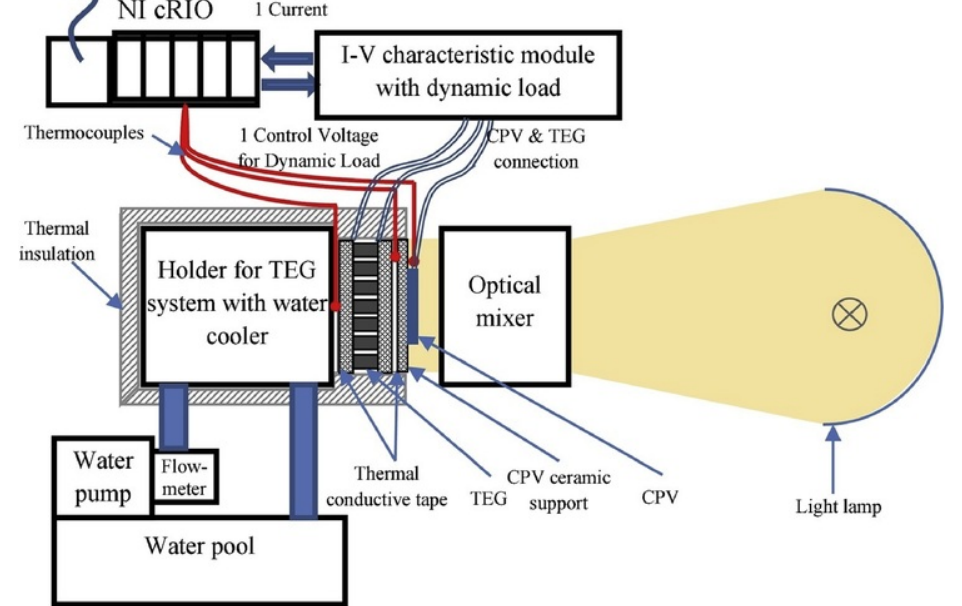

Fig. 15 Schematic diagram of experimental PV/TEG setup [78].

alt-text: Fig. 15

\subsection{Hybrid system coupling}

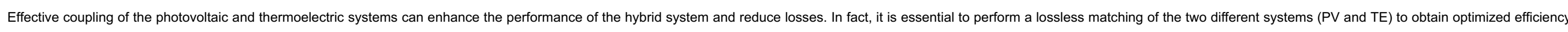
results. In addition, a TEG possesses an internal resistance which must be adequately matched with that of the PV so as the ensure the hybrid system performance is not worse than that of the individual systems.

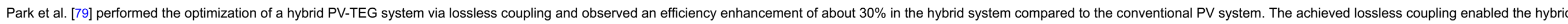

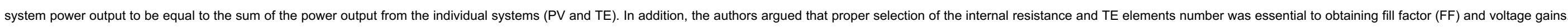

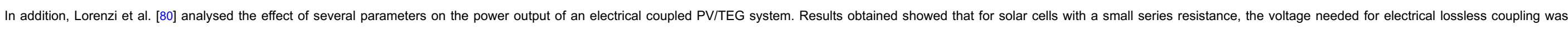
smaller. In addition, it was found that lossless conditions strong depend on temperature.

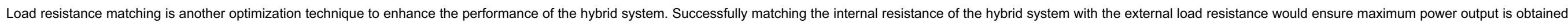

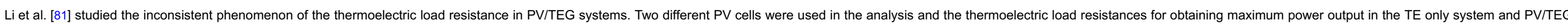

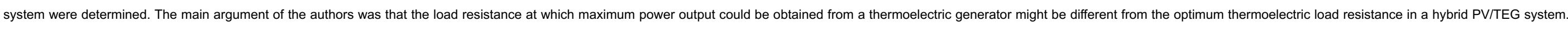

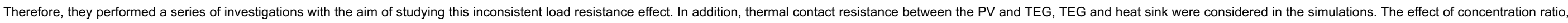

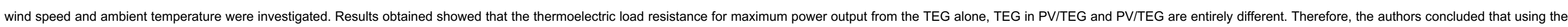
optimum TE load resistance in a TEG only system for the analysis of a PV/TEG system would cause errors and prevent the attainment of hybrid system maximum power output.

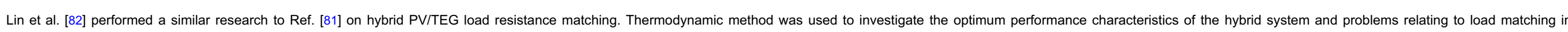

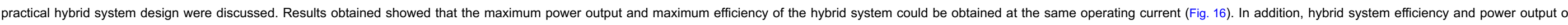
about $27 \%$ compared to the PV only system were observed. Finally, the authors argued that the use of thermoelectric material with a figure of merit $(Z=0.004 / K)$ could lead to the hybrid system efficiency enhancement of about $30 \%$. 

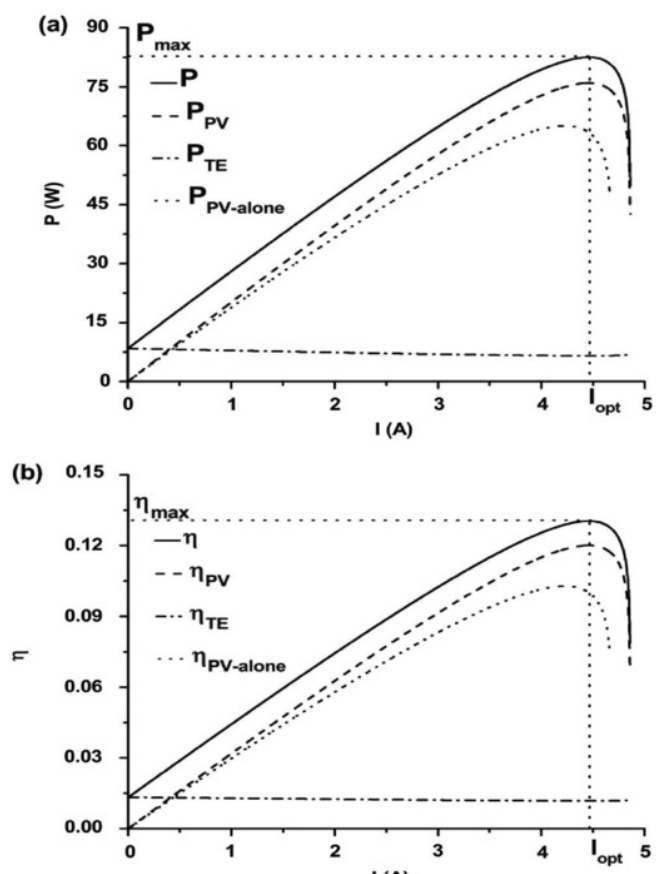

I(A)

Fig. 16 Hybrid system and PV only system (a) power output and (b) efficiency [82]

alt-text: Fig. 16

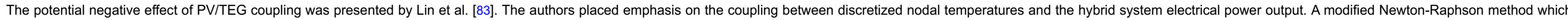

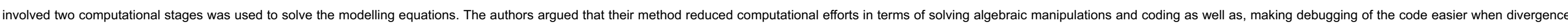

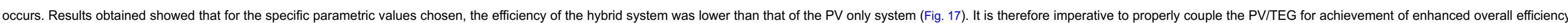
rather than reduced efficiency when compared to the PV only system.

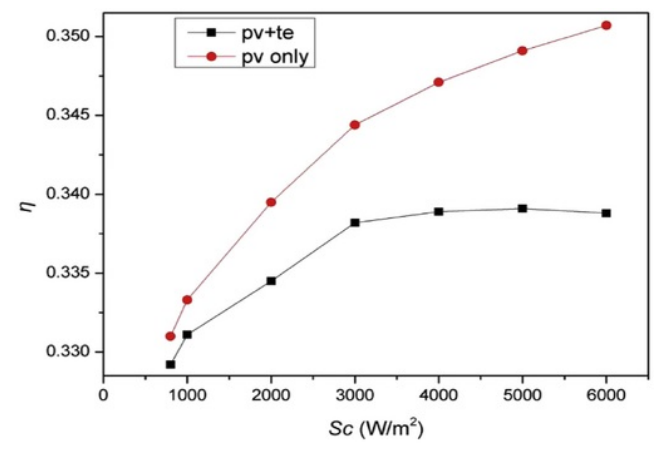

Fig. 17 Variation of hybrid system and PV only system efficiency with solar radiation [83].

alt-text: Fig. 17

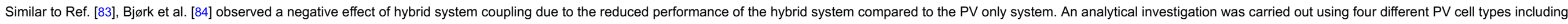

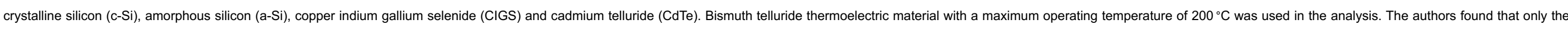



power production due to the decrease in PV performance as temperature increased.

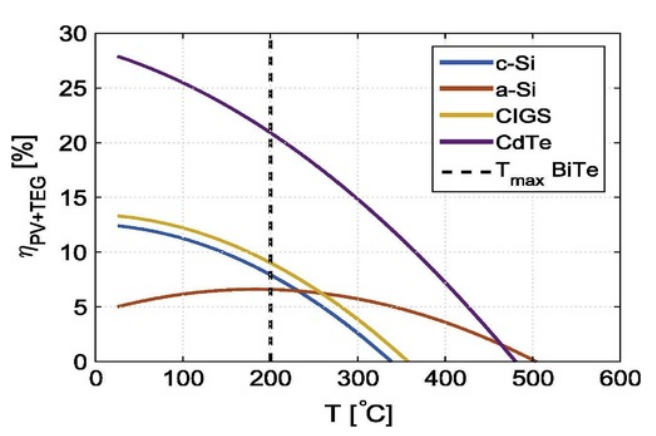

Fig. 18 Hybrid system efficiency variation with temperature for different PV cells [84]. alt-text: Fig. 18

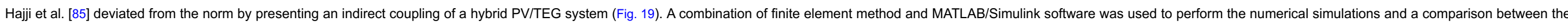

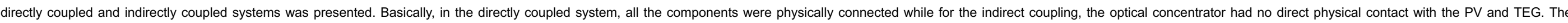
developed system was properly insulated to reduce heat loss and it was observed that the indirect coupling method significantly improved the hybrid system overall efficiency.

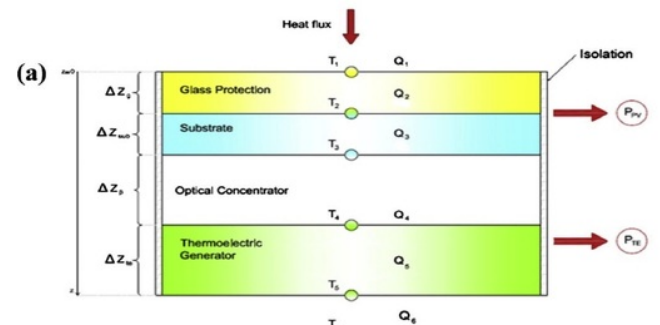

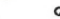

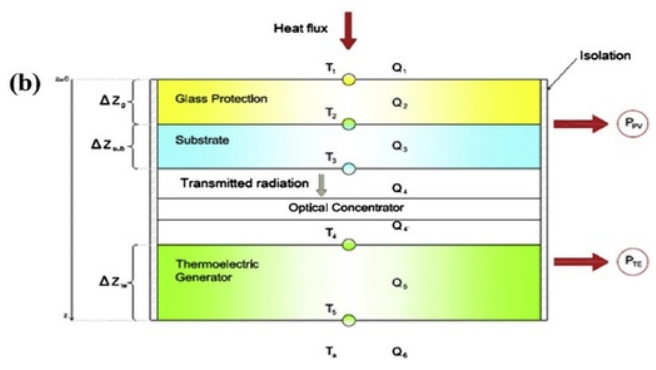

Fig. 19 Schematic of PV/TEG system for (a) direct and (b) indirect coupling [85]. alt-text: Fig. 19 


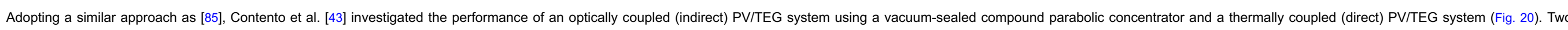

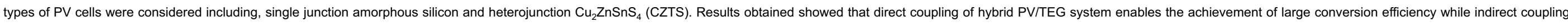

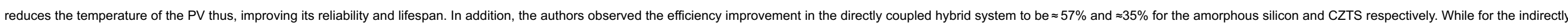
coupled hybrid system, the efficiency improvement was $\approx 42 \%$ and $\approx 24 \%$ for the amorphous silicon and CZTS respectively.

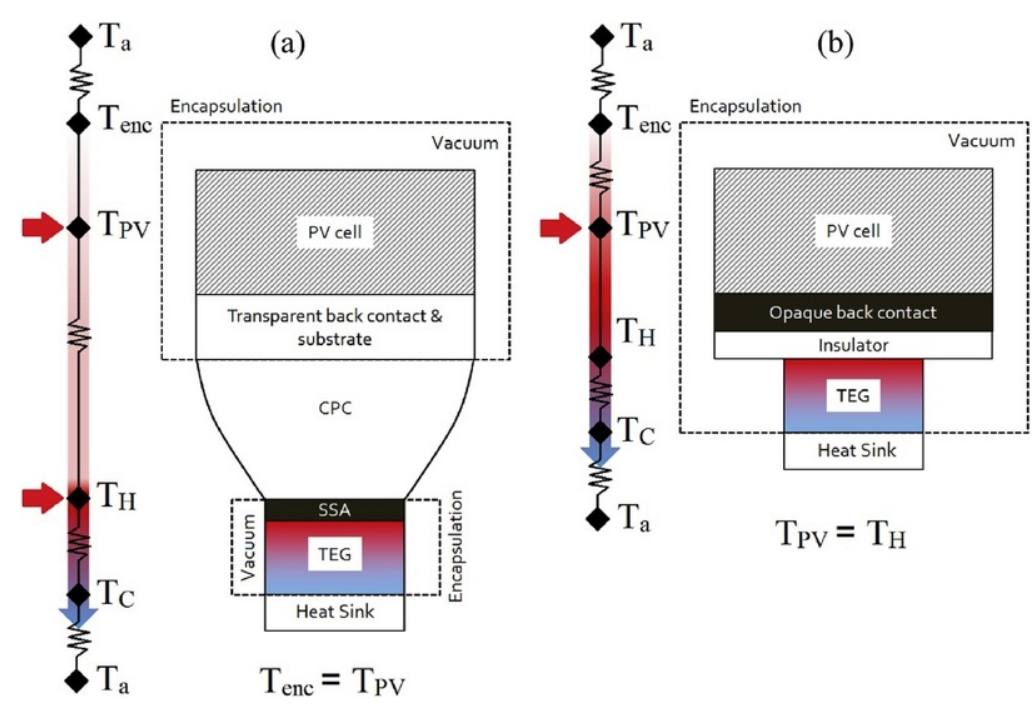

Fig. 20 Schematic of developed hybrid system with (a) indirect and (b) direct coupling [43].

\section{alt-text: Fig. 20}

\subsection{Thermoelectric geometry optimization}

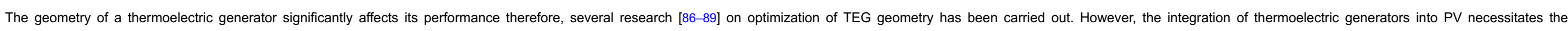
investigation of the optimum geometry of the TEG in the hybrid PV/TEG system as this may differ from the optimum geometry in the TEG only system.

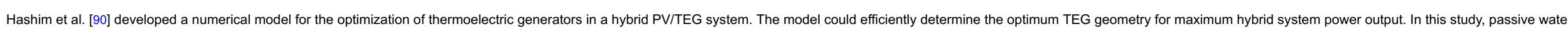

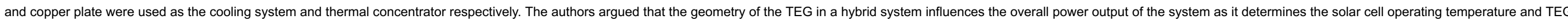

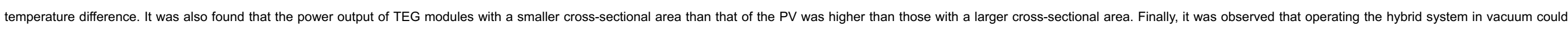
enhance its performance.

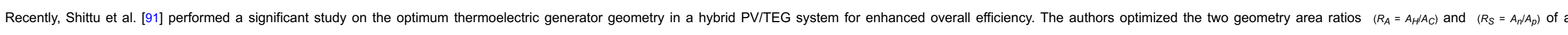

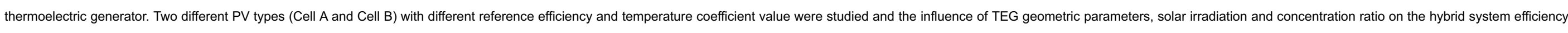

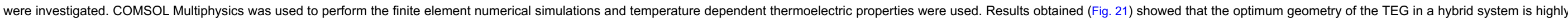
dependent on the type of solar cell used. 

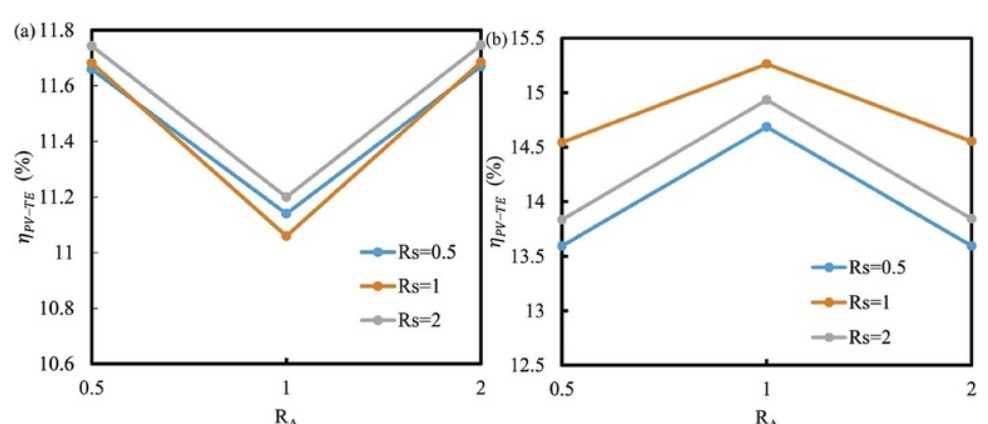

Fig. 21 Hybrid system efficiency variation with geometry area ratio for (a) solar cell A and (b) solar cell [91] alt-text: Fig. 21

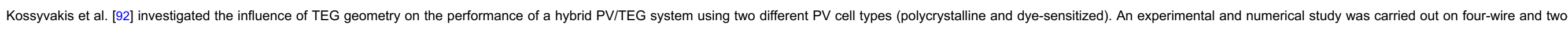

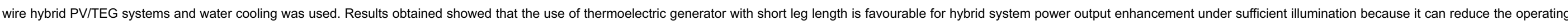
temperature of the PV cell. This observation is significant because it can reduce the hybrid system manufacturing costs because less thermoelectric material will be used to achieve high overall power output.

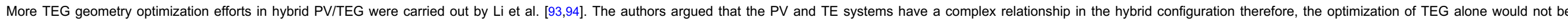

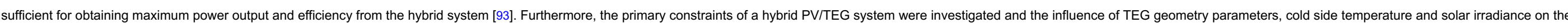
performance of the hybrid system were studied. It was found that the overall efficiency of the hybrid system increased as the cross-sectional area of the TEG increased and shorter TEG leg length is favourable [94].

\subsection{Energy storage}

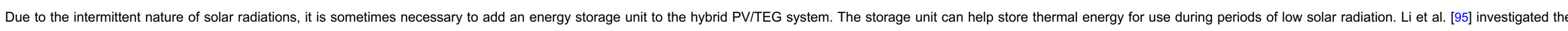

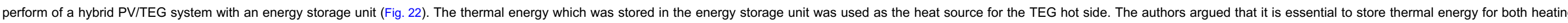

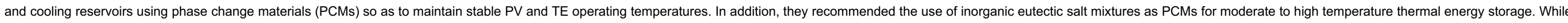

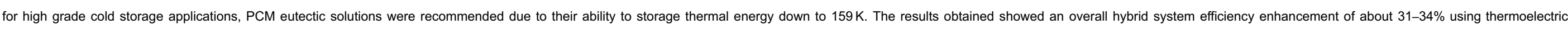
materials with ZT $=1$ (see Fig. 24) (see Fig. 23)

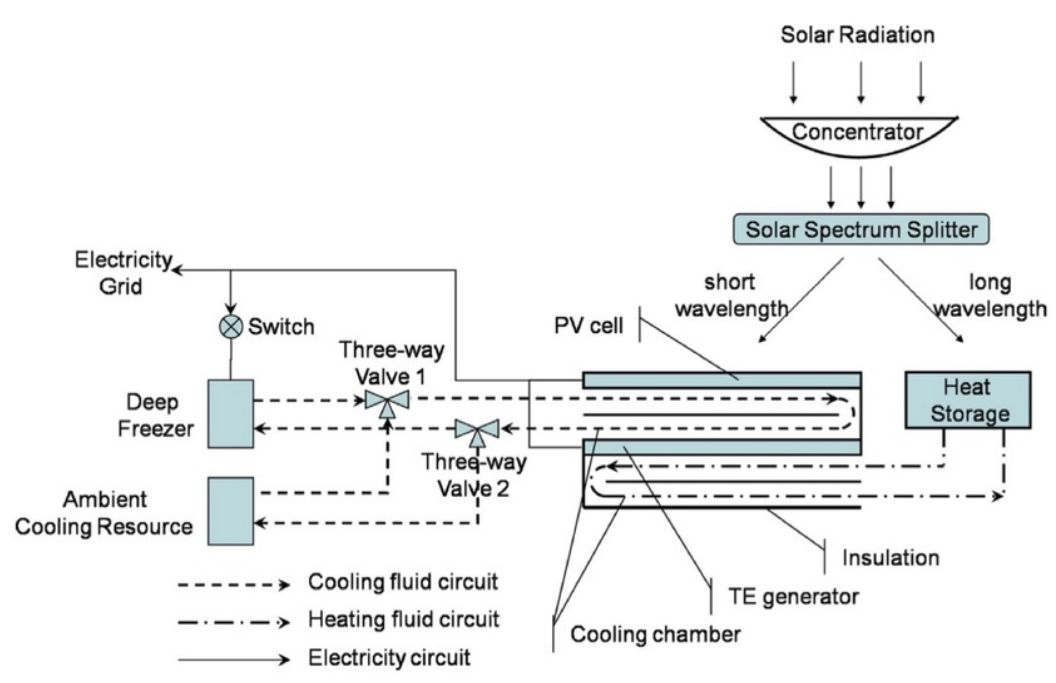




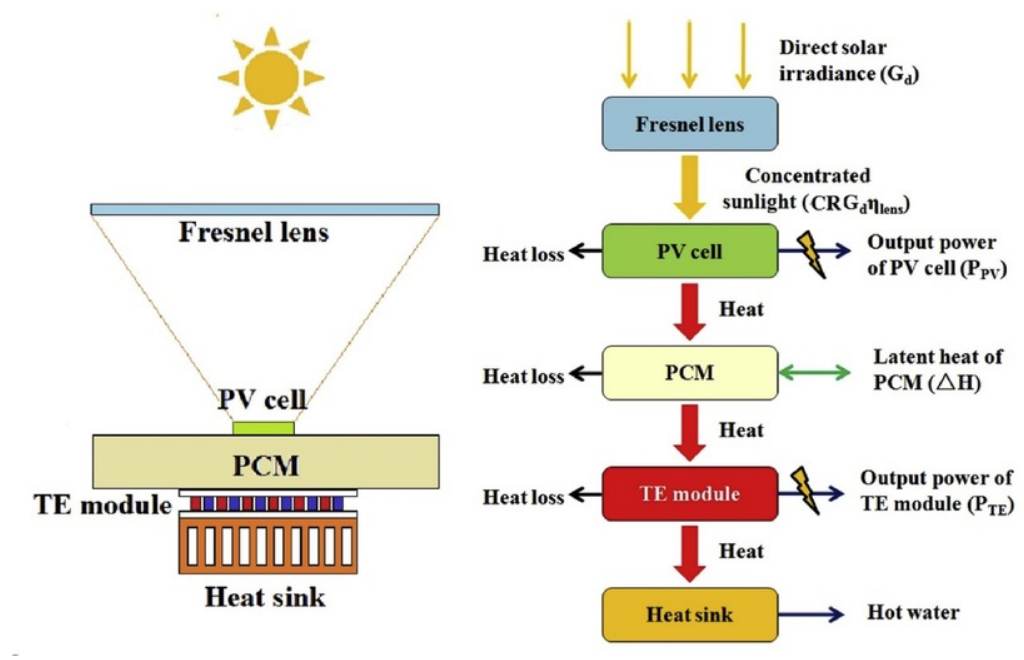

Fig. 23 Schematic diagram and energy flow of the hybrid PV-PCM-TE system [97].

alt-text: Fig. 23

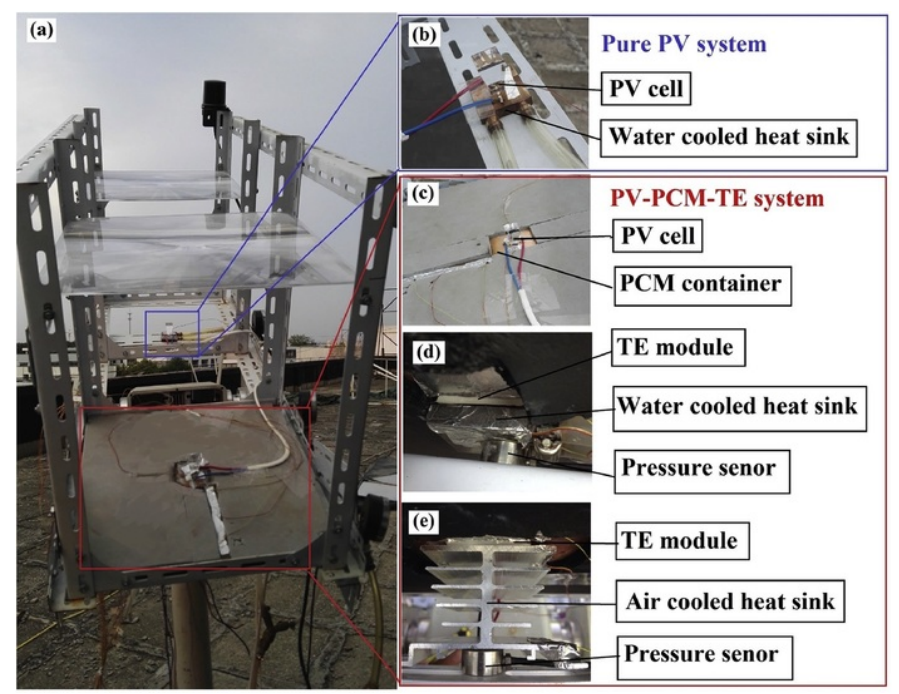

Fig. 24 (a) Hybrid PV-PCM-TE system and PV only system, (b) PV only system, (c) top view of hybrid PV-PCM-TE, (d) bottom view of hybrid system with water heat sink and (3) bottom view of hybrid system with air heat sink [97]. alt-text: Fig. 24

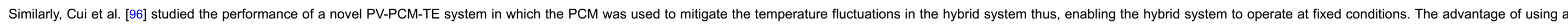

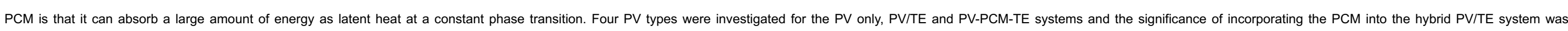

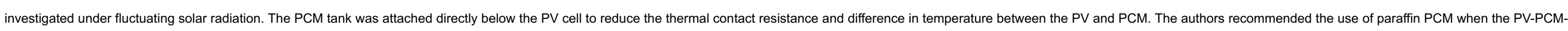




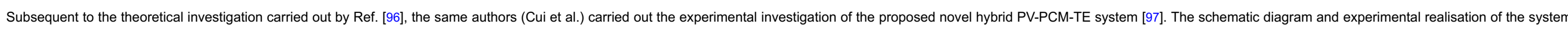

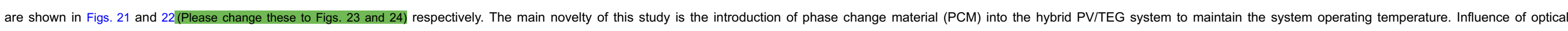

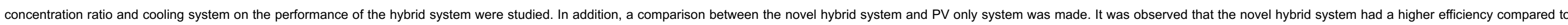
the PV only system due to the use of phase change material.

\subsection{Thermoelectric generator cooling}

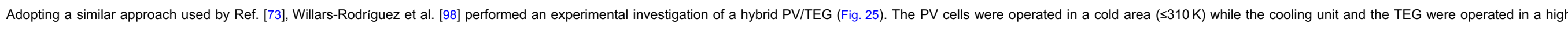

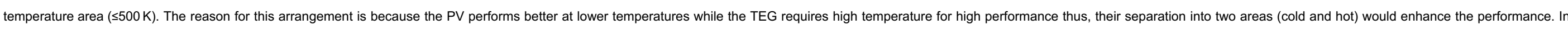

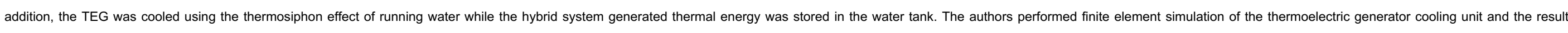

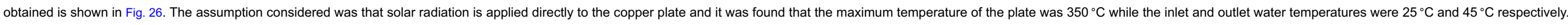
Results obtained showed that the hybrid system generated an electric power of $7 \mathrm{~W}$ and thermal power of $30 \mathrm{~W}$.

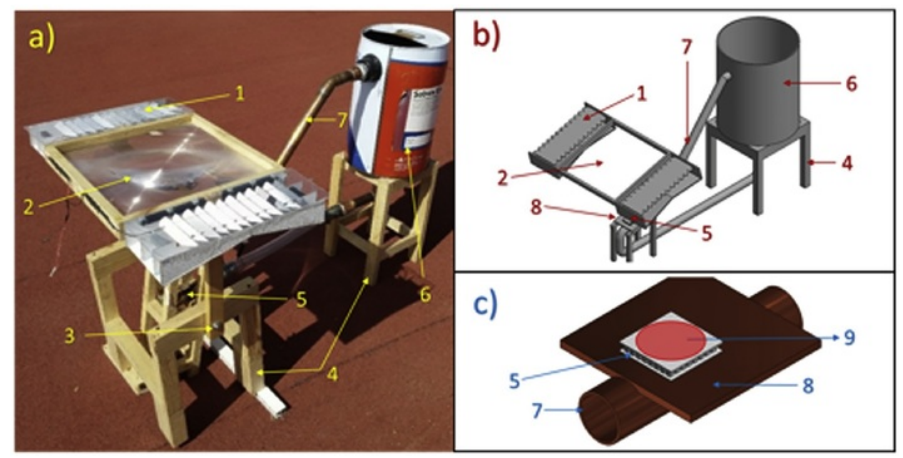

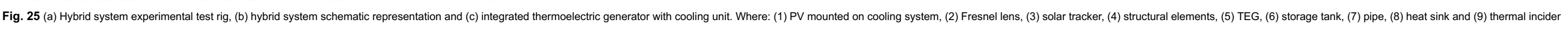
spot [98].

\section{alt-text: Fig. 25}

(a)

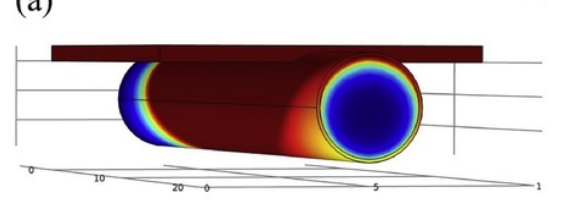

Temperature (degC) (b)

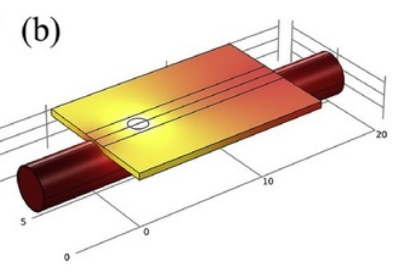

$\hat{L}_{\rightarrow \rightarrow 2} .268$

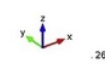

Fig. 26 Temperature distribution in thermoelectric generator cooling unit (a) running water tube and (b) copper plate [98].

alt-text: Fig. 26

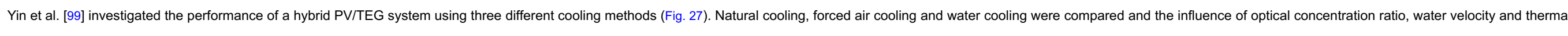

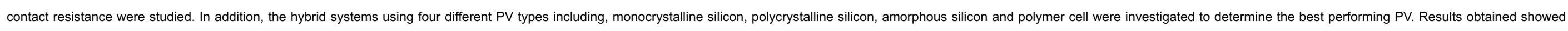




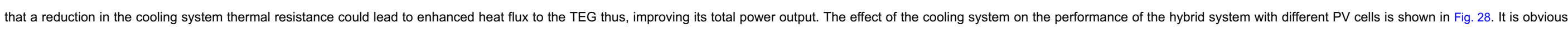

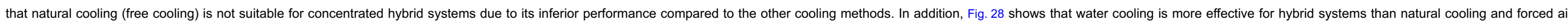

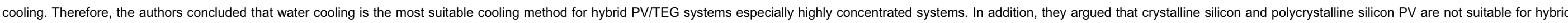
PV/TEG systems because of their reduced overall efficiency compared to the PV only system.

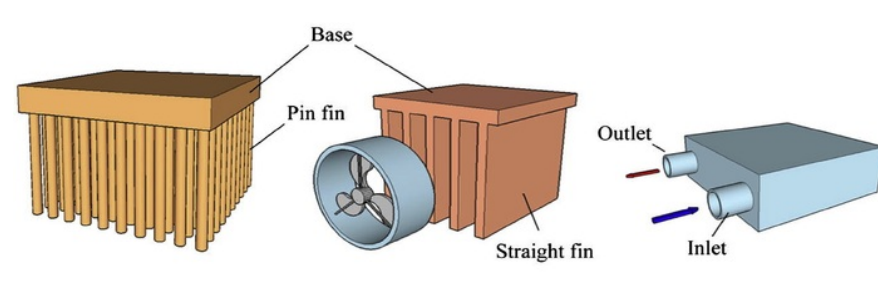
Free cooling
Forced air cooling
Water cooling

Fig. 27 Different types of thermoelectric generator cooling systems [99].

\section{alt-text: Fig. 27}

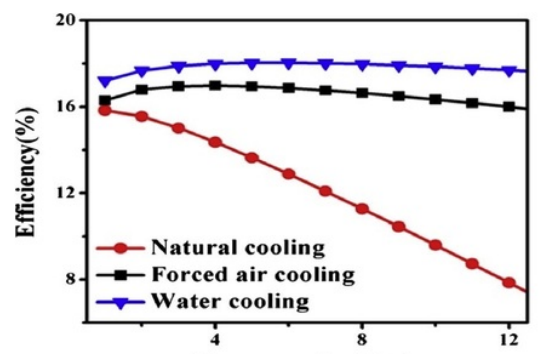

(a)

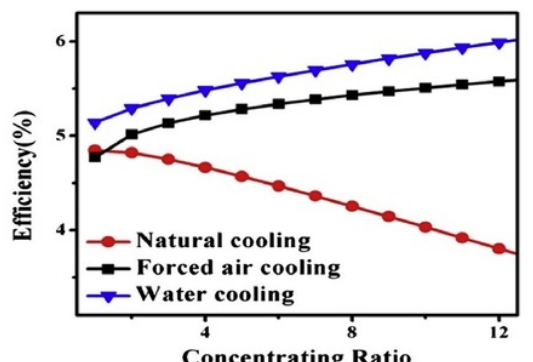

ting Ratio
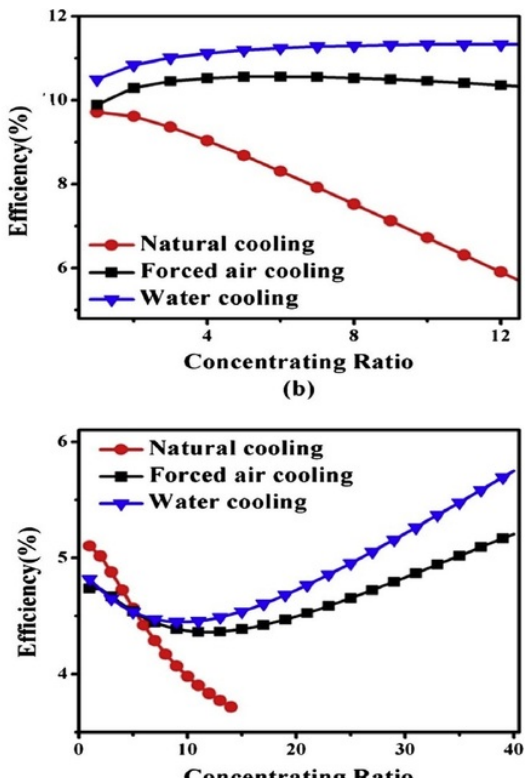

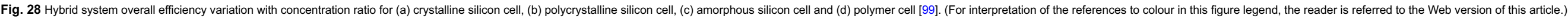
alt-text: Fig. 28

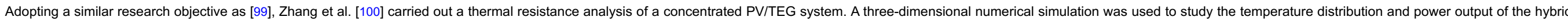

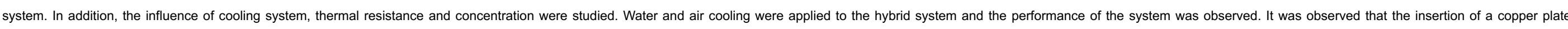

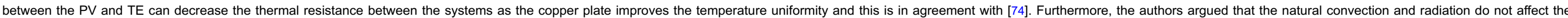

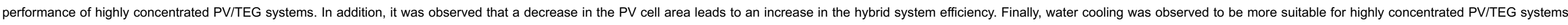
compared to air cooling. This finding is in agreement with [99].

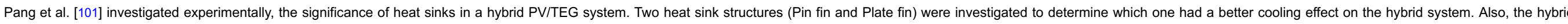




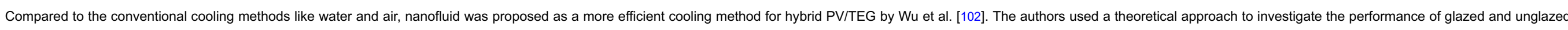

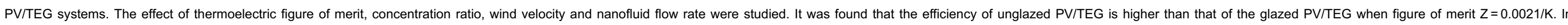

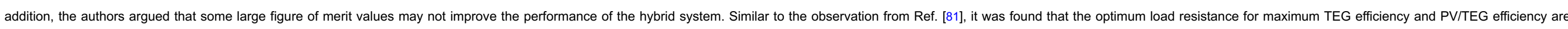
different. Finally, the results obtained showed that nanofluid cooling enhances the performance of PV/TEG systems compared to water cooling especially for the glazed system (Fig. 29).

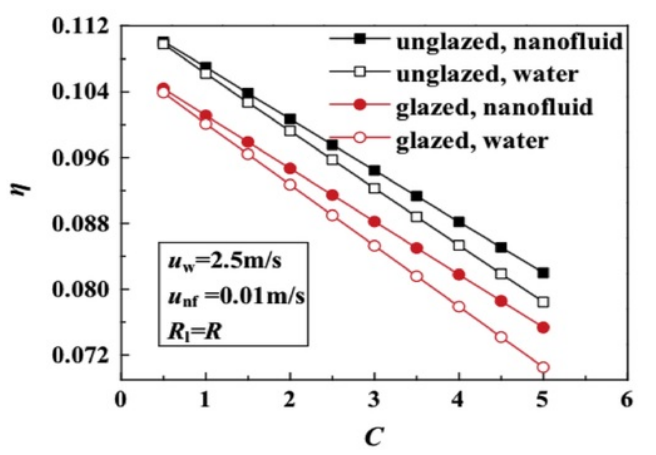

Fig. 29 Variation of hybrid system efficiency with concentration ratio for different cooling systems [102]

\section{alt-text: Fig. 29}

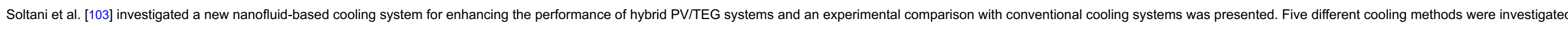

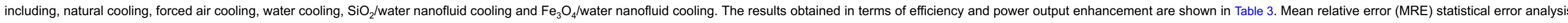

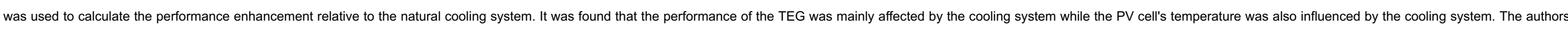
argued that nanofluid cooling performed better especially $\mathrm{SiO}_{2} /$ water nanofluid cooling which enhanced the efficiency and power output of the hybrid system by $3355 \%$ and $8.26 \%$ respectively compared to the natural cooling.

\subsection{New generation PV and TE materials}

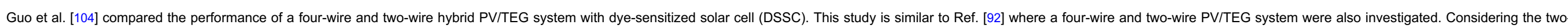

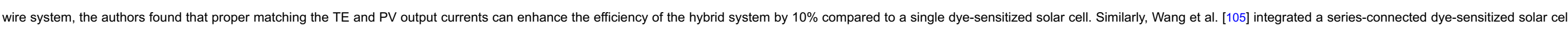

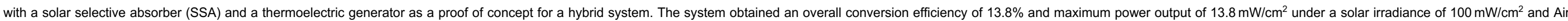
Mass of 1.5G. The authors argued that the conversion efficiency of the hybrid system could be enhanced further by optimizing the system.

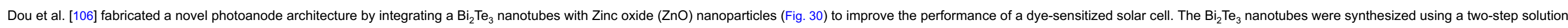

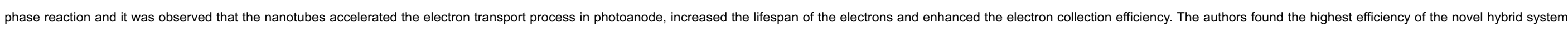
composed of $\mathrm{DSCC}$ and $\mathrm{Bi}_{2} \mathrm{Te}_{3}(1.5 \mathrm{wt} \%)$ to be $4.27 \%$ which was $44.3 \%$ higher than that of a single $\mathrm{ZnO}$ photoanode. 


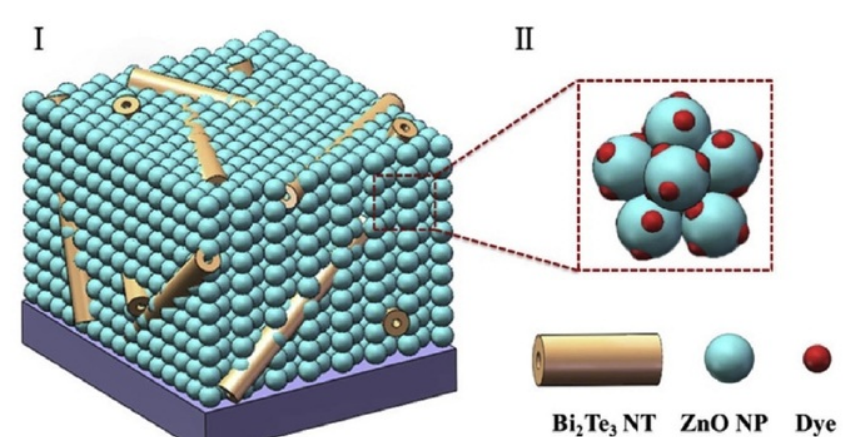

III

$\mathrm{Bi}_{2} \mathrm{Te}_{3} \mathrm{NT}$ ZnO NP Dye

IV

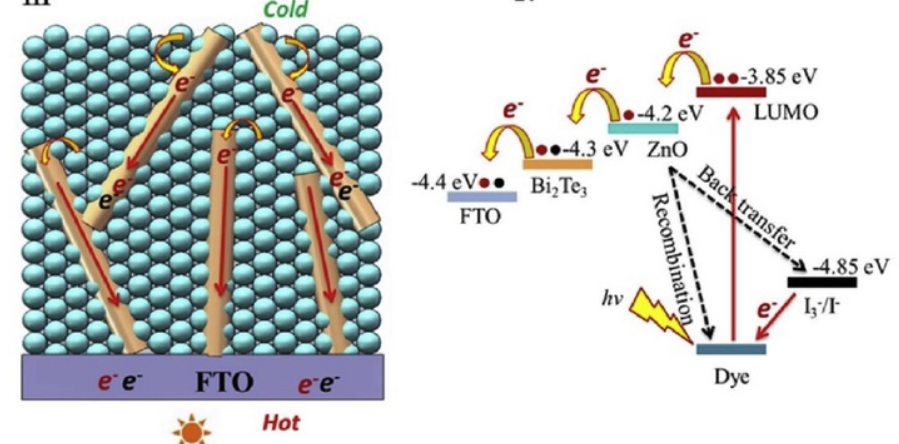

$\rightarrow$ Electron transport path $\bullet$ Photoelectric conversion - Thermoelectric conversion

Fig. 30 Schematic diagram of $\mathrm{Bi}_{2} \mathrm{Te}_{3} / \mathrm{ZnO}$ composite photoanode (I, II) electron transport process, (III, IV) energy diagram of system operation [106]

alt-text: Fig. 30

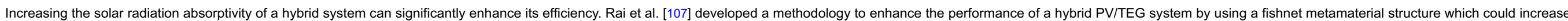

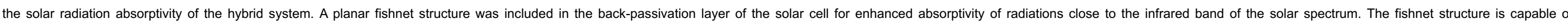

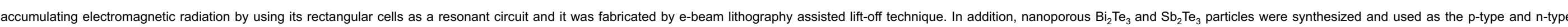

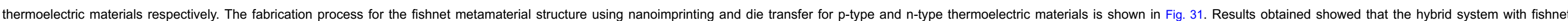
structure had an enhanced efficiency around 11 folds.

Fishnet pattern

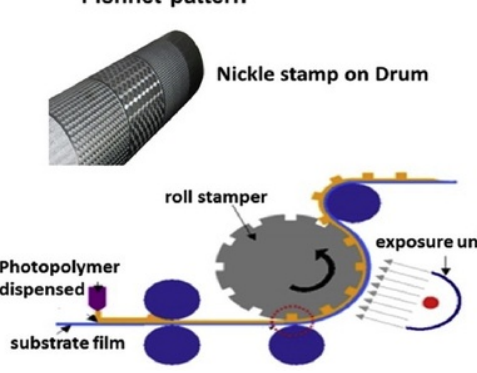

p-type and n-type TE material mount

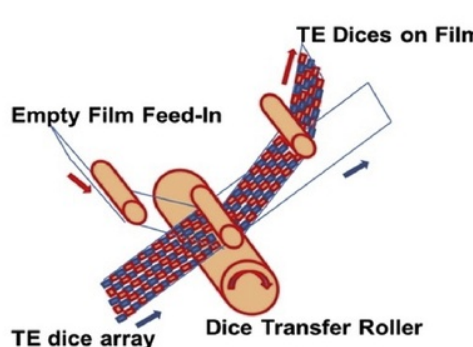

Fig. 31 Fabrication process for fishnet metamaterial structure [107]

alt-text: Fig. 31 


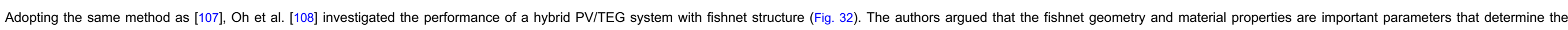

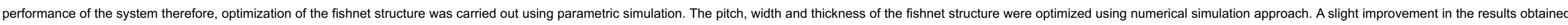
from Ref. [107] was observed by the authors [108] as the optimized hybrid system with fishnet structure had an enhanced efficiency around 12 folds compared to the system without fishnet.

(a)

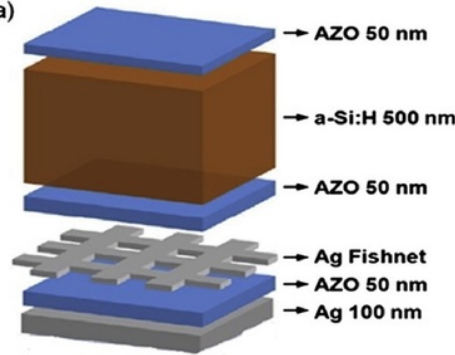

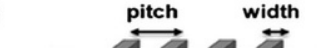

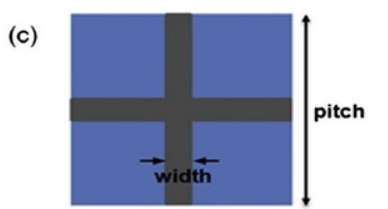

Fig. 32 Schematic diagram of hybrid stem with fishnet. (a) 3D schematic of thin film solar cell, (b) fishnet structural parameters and (c) top view of solar cell [108]. alt-text: Fig. 32

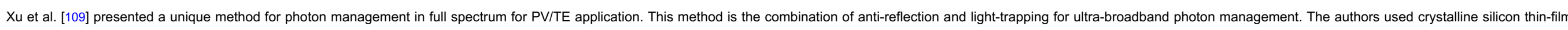

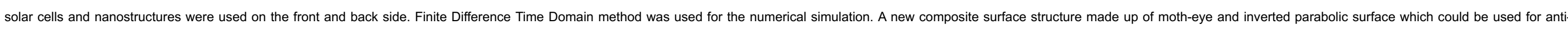

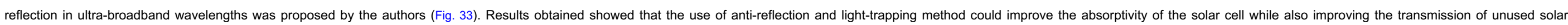
radiation to the thermoelectric generator. Therefore, the performance of the hybrid PV/TEG could be enhanced.

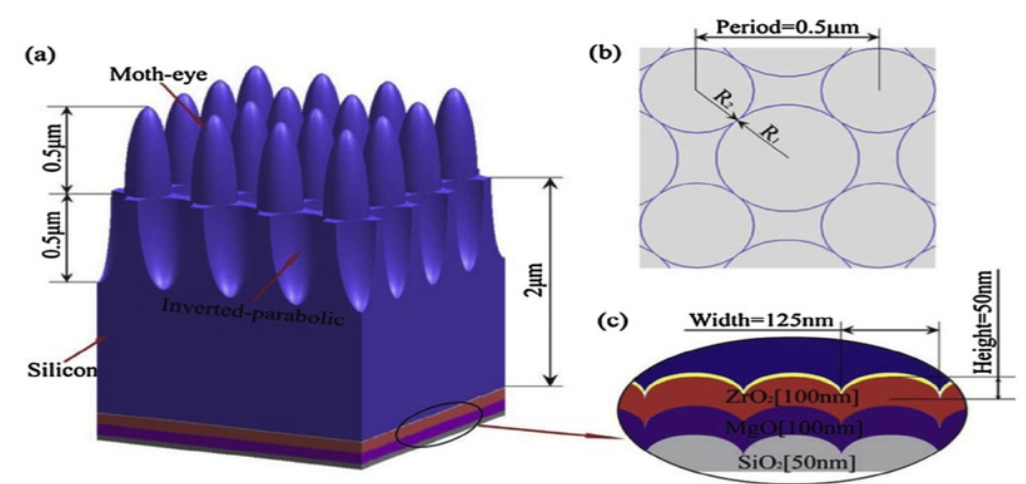

Fig. 33 (a) Silicon solar cell structure, (b) top view of solar cell structure and (c) cross-view of bottom antireflection coating [109]. (For interpretation of the references to colour in this figure legend, the reader is referred to the Web version of this article.) alt-text: Fig. 33

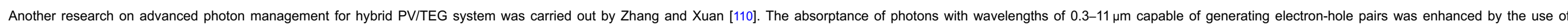

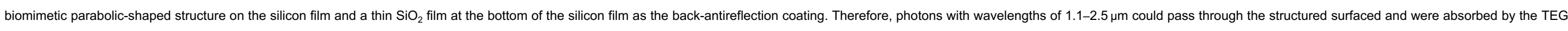
thus, the overall efficiency of the hybrid system was increased. Finally, the authors argued that the use of omnidirectional broadband photon management can enhance the performance of hybrid systems.

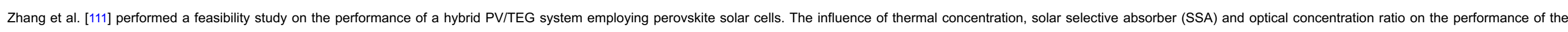

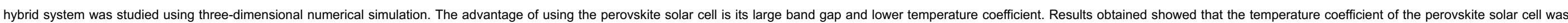

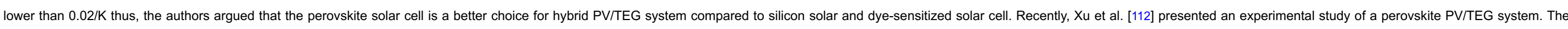

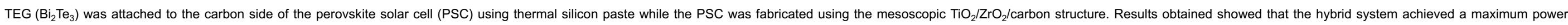




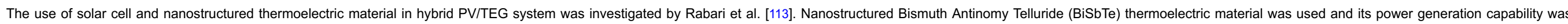

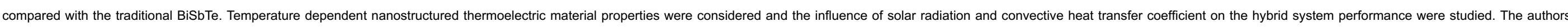
argued that the nanostructured thermoelectric material enhanced the performance of the TEG.

\subsection{Maximum power tracking and control system}

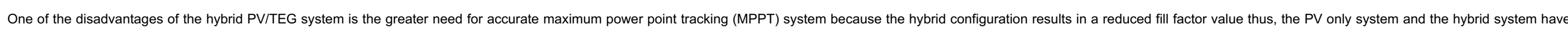

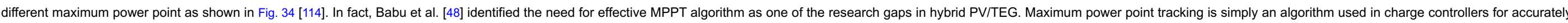

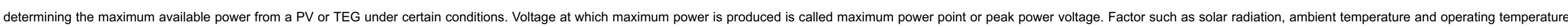
influence the maximum power point.

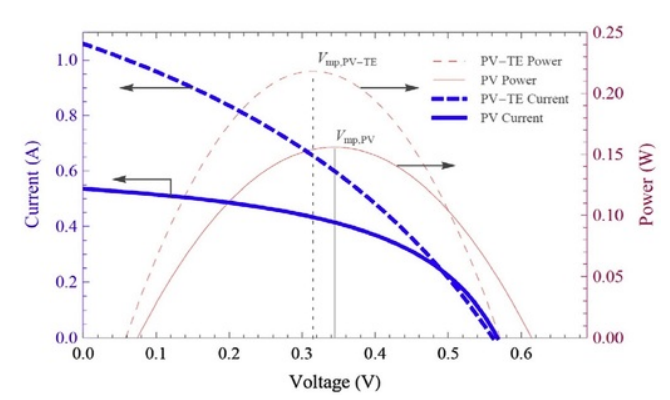

Fig. 34 PV/TEG and PV current-voltage characteristics [114].

alt-text: Fig. 34

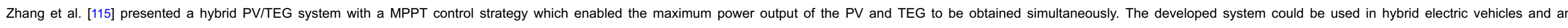

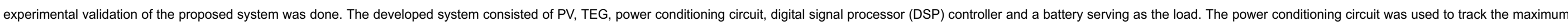

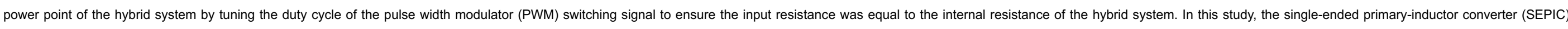
circuit was used.

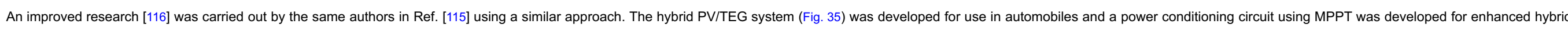

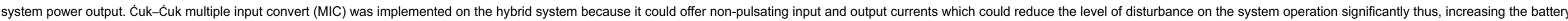

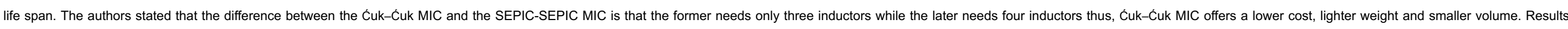
obtained showed that the overall hybrid system power output was enhanced by the Ćuk-Ćuk MIC using the MPPT.

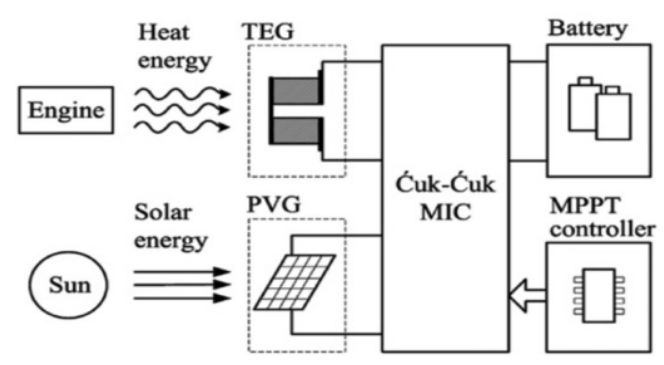

Fig. 35 Hybrid PV/TEG system with the Ćuk-Ćuk MIC [116] 


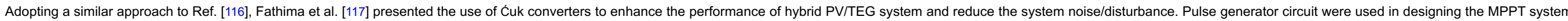

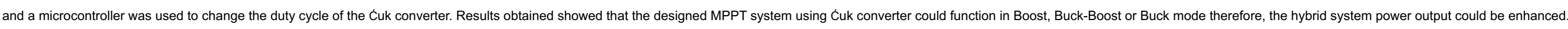

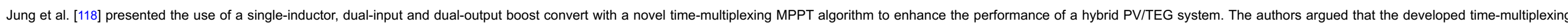

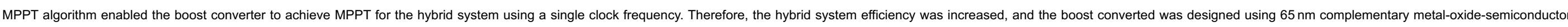
(CMOS) technology. Finally, a peak efficiency of $78 \%$ was obtained by the hybrid system.

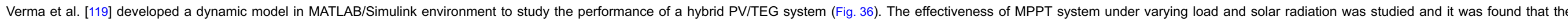

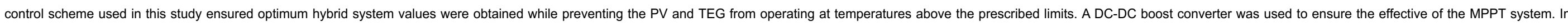

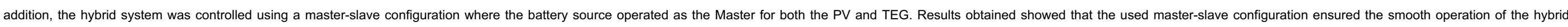
system.

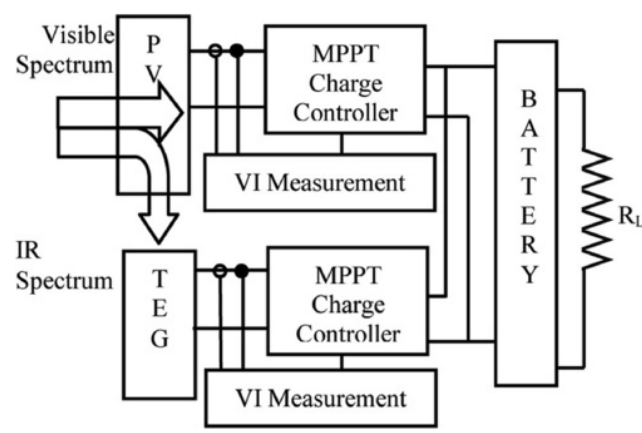

Fig. 36 Proposed hybrid system with MPPT for waste heat recovery [119].

alt-text: Fig. 36

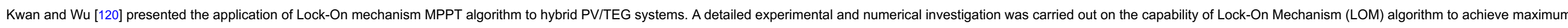

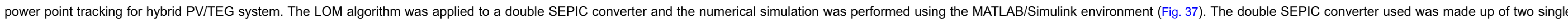

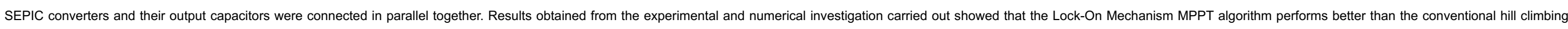
algorithm. 


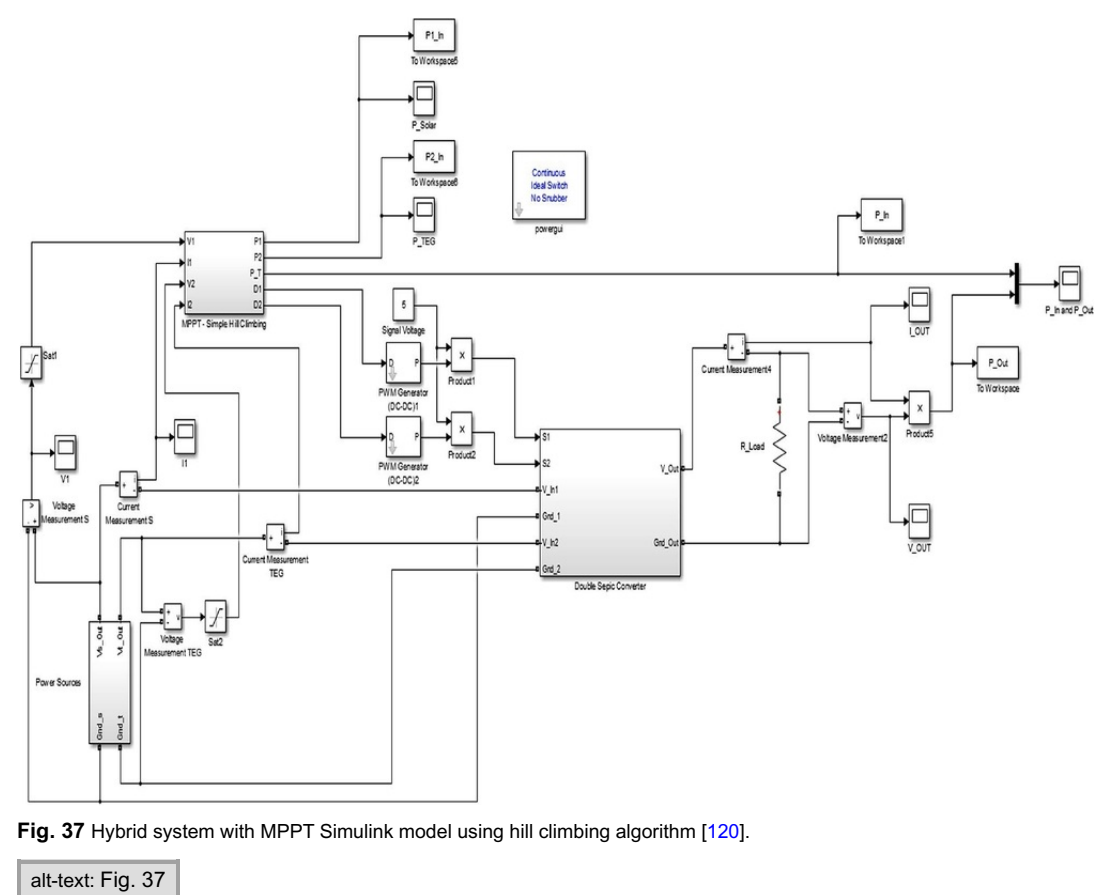

\subsection{Niche applications}

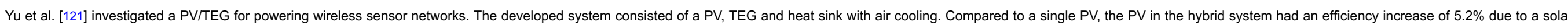

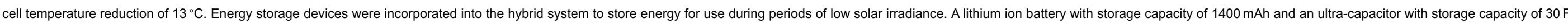
were used to store energy from the PV and TEG respectively. The developed hybrid system had the capacity to renew energy by itself thus it could provide reliable and long-time power to the sensor node.

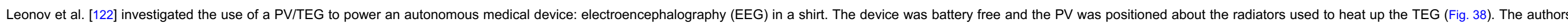

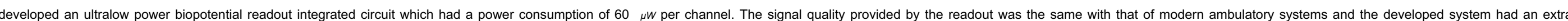
advantage of being wireless compared to wired commercial systems thus, the biopotential signals could be transmitted to a doctor in real time.
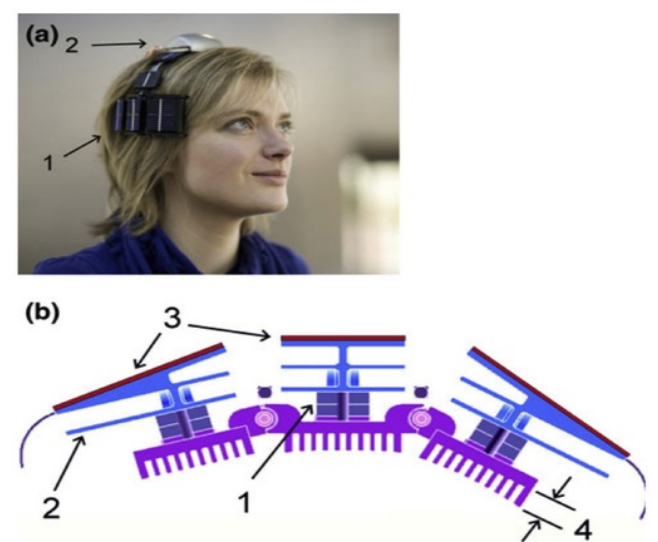


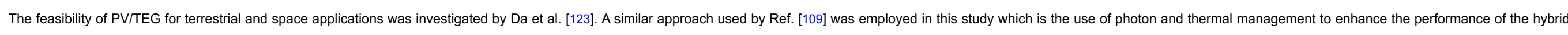

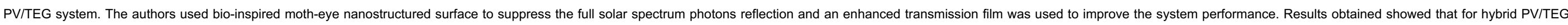

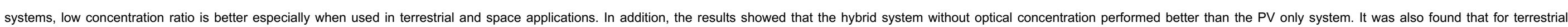

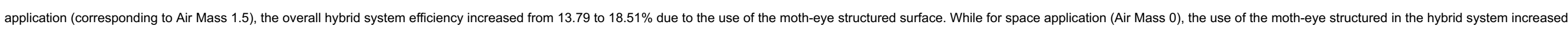
the efficiency to $16.84 \%$ (Fig. 39).
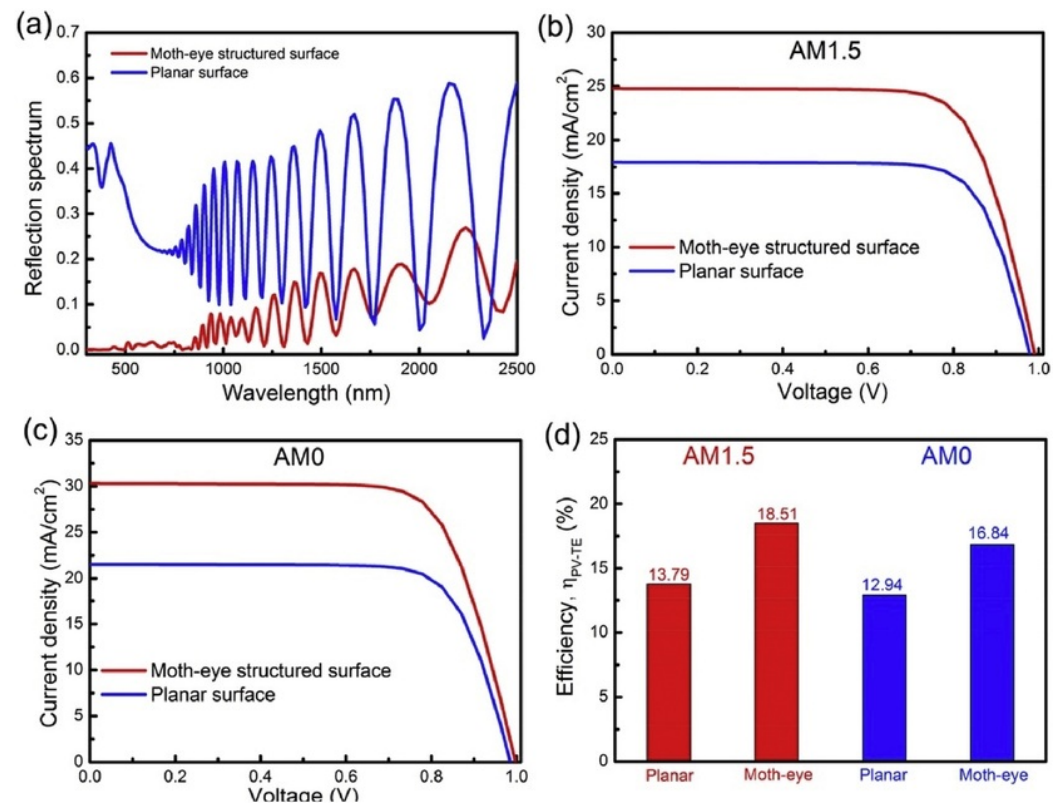

\begin{tabular}{|l|} 
Fig. 39 Performan \\
\hline alt-text: Fig. 39 \\
\hline
\end{tabular}

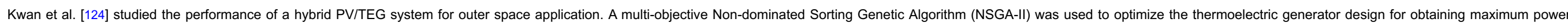

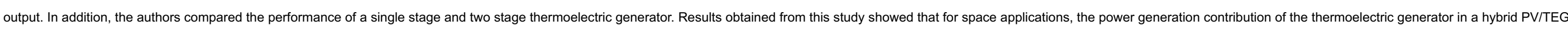

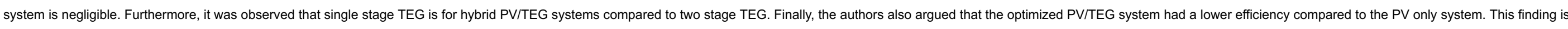
in agreement with other similar findings like $[83,84]$.

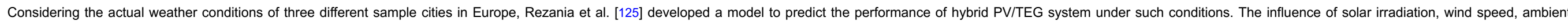

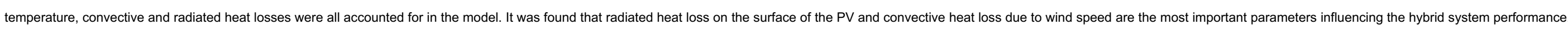

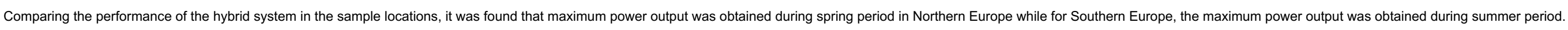

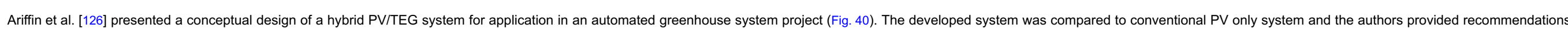

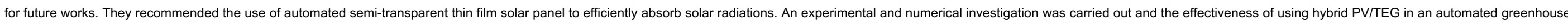
system was demonstrated. 


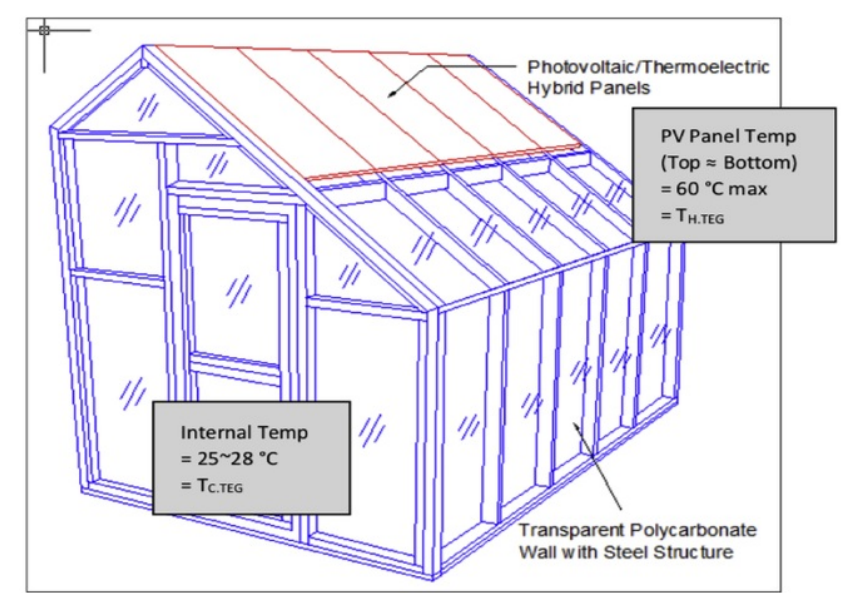

Fig. 40 Conceptual design of a hybrid PV/TEG for automated greenhouse system [126]. alt-text: Fig. 40

\section{Discussion and recommendations}

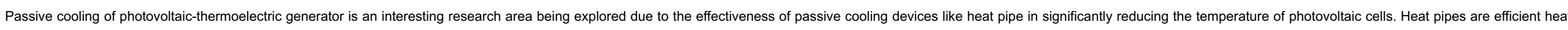

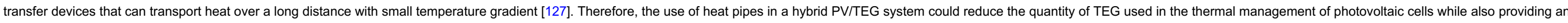

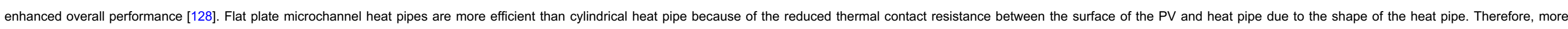
research on the integration of hybrid PV/TEG with flat plate microchannel heat pipes are strongly recommended especially because of the encouraging results reported from such heat pipe hybrid systems by Refs. [127,128].

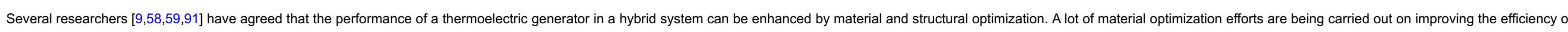

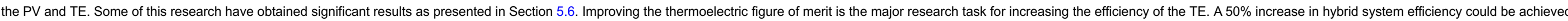

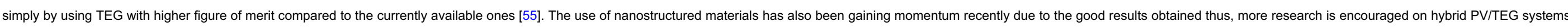

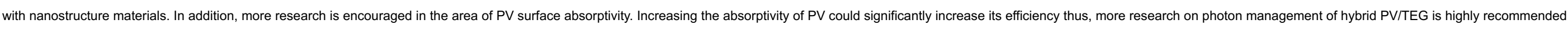

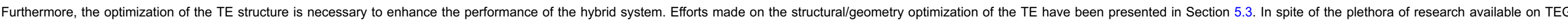

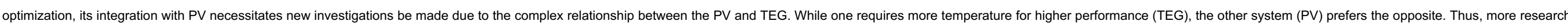

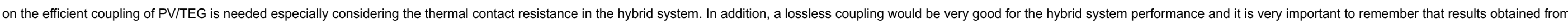
TEG only optimization is not sufficient for the hybrid system optimization. Therefore, the optimized load resistance and geometry of the TEG in a TEG only system is different from the one in a hybrid system due to the influence of the PV.

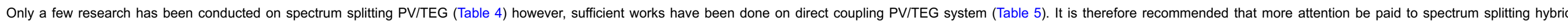

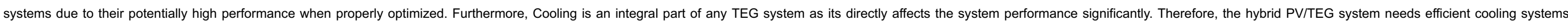

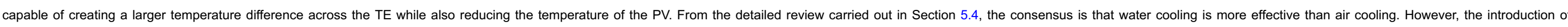

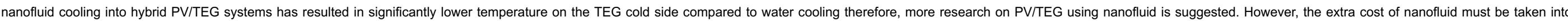
consideration and a justification must be made in terms of overall performance compared to hybrid systems with cheap conventional cooling.

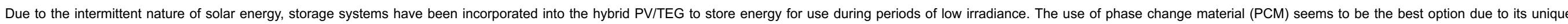

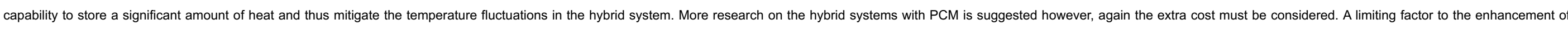
hybrid system performance is the need for opportunity cost analysis. While there are obvious ways to easily improve the performance of the system, a trade-off must be made due to the high cost of such optimization.

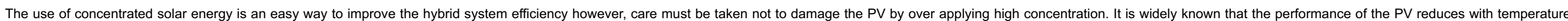




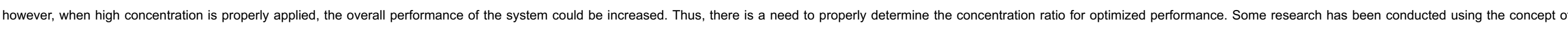

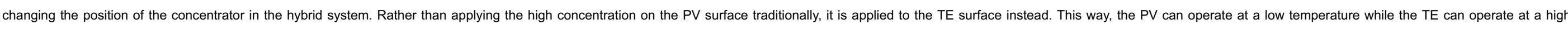

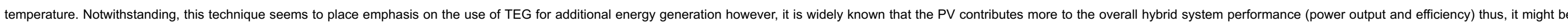

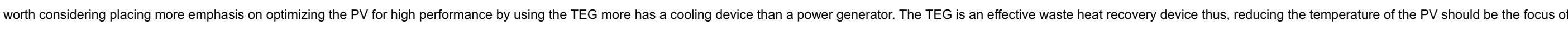
hybrid PV/TEG system optimization.

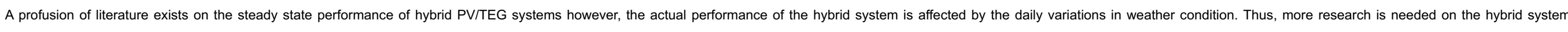

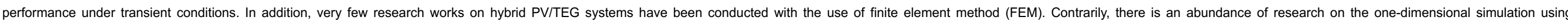

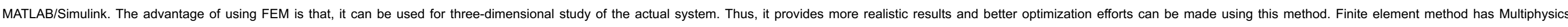

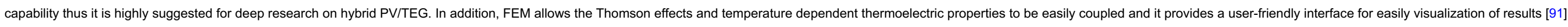

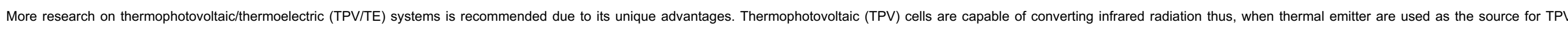

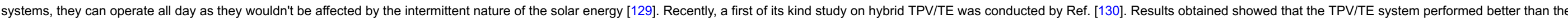

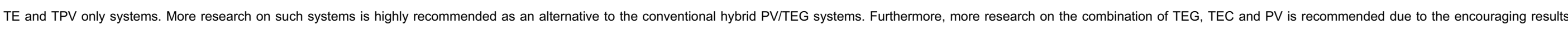

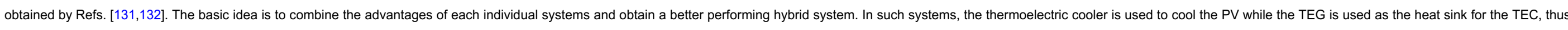

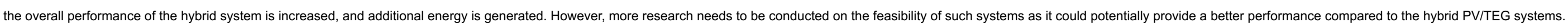

\section{Conclusion}

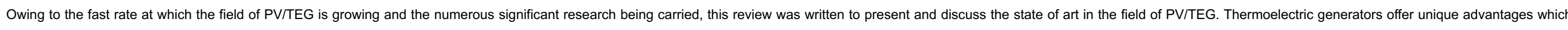

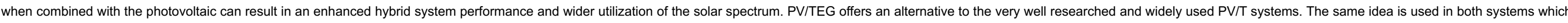

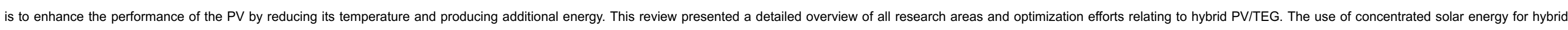

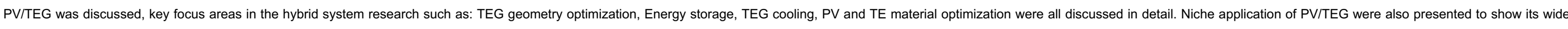

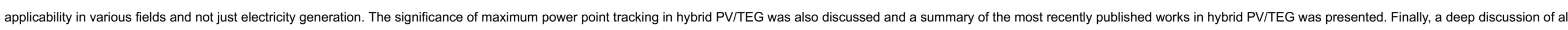

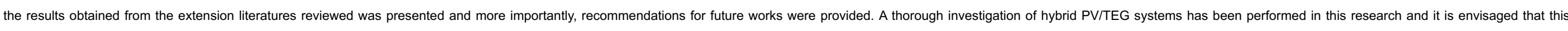
review would serve as an indispensable literature on hybrid PV/TEG.

\section{Acknowledgement}

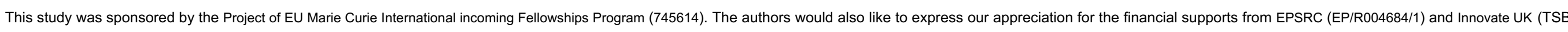

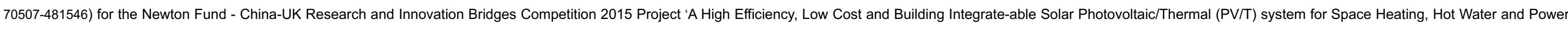
Supply' and DongGuan Innovation Research Team Program (No. 2014607101008).

\section{References}

[1] R.K. Akikur, R. Saidur, H.W. Ping and K.R. Ullah, Comparative study of stand-alone and hybrid solar energy systems suitable for off-grid rural electrification: a review, Renew Sustain Energy Rev 27, 2013, 738-752,

https://doi.org/10.1016/j.rser.2013.06.043.

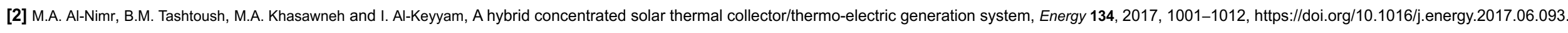

[3] M. Thirugnanasambandam, A. Review of solar thermal technologies, Renew Sustain Energy Rev 14, 2010, 312-322, https://doi.org/10.1016/j.rser.2009.07.014.

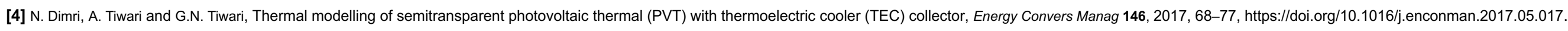

[5] A. Makki, S. Omer and H. Sabir, Advancements in hybrid photovoltaic systems for enhanced solar cells performance, Renew Sustain Energy Rev 41, 2015, 658-684, https://doi.org/10.1016/j.rser.2014.08.069. 
[6] F. Grubisić-Čabo, S. Nizetić and T.G. Marco, Photovoltaic panels: a review of the cooling techniques, Trans FAMENA 40, 2016, 63-74, https://doi.org/10.1017/S0020818300000849.

[7] W. He, G. Zhang, X. Zhang, J. Ji, G. Li and X. Zhao, Recent development and application of thermoelectric generator and cooler, Appl Energy 143, 2015, 1-25 https://doi.org/10.1016/j.apenergy.2014.12.075.

[8] G. Li, S. Shittu, T.M.O. Diallo, M. Yu, X. Zhao and J. Ji, A review of solar photovoltaic-thermoelectric hybrid system for electricity generation, Energy 158, 2018, 41-58, https://doi.org/10.1016/j.energy.2018.06.021.

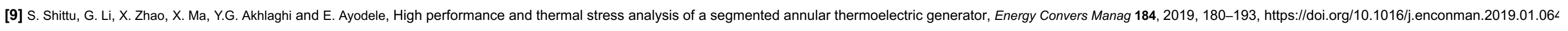

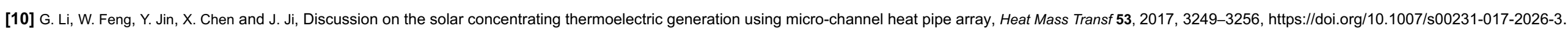

[11] D. Narducci, P. Bermel, B. Lorenzi and N. Wang, Hybrid and fully thermoelectric solar harvesting, first ed., 2018, Springer International Publishing; Switzerland, https://doi.org/10.1109/IPDPS.2006.1639640.

[12] D.M. Chapin, C.S. Fuller and G.L. Pearson, A new silicon p-n junction photocell for converting solar radiation into electrical power [3], J Appl Phys 25, 1954, 676-677, https://doi.org/10.1063/1.1721711.

[13] L. El Chaar, L.A. Lamont and N. El Zein, Review of photovoltaic technologies, Renew Sustain Energy Rev 15, 2011, 2165-2175, https://doi.org/10.1016/j.rser.2011.01.004

[14] P.G.V. Sampaio, M.O.A. González, R.M. de Vasconcelos, M.A.T. dos Santos, J.C. de Toledo and J.P.P. Pereira, Photovoltaic technologies: mapping from patent analysis, Renew Sustain Energy Rev 93, 2018, 215-224, https://doi.org/10.1016/j.rser.2018.05.033.

[15] P. Huen and W.A. Daoud, Advances in hybrid solar photovoltaic and thermoelectric generators, Renew Sustain Energy Rev 72, 2017, 1295-1302, https://doi.org/10.1016/j.rser.2016.10.042.

[16] M.A. Green, Y. Hishikawa, E.D. Dunlop, D.H. Levi, J. Hohl-Ebinger and A.W.Y. Ho-Baillie, Solar cell efficiency tables (version 52), Prog Photovoltaics Res App/ 26, 2018, 427-436, https://doi.org/10.1002/pip.3040.

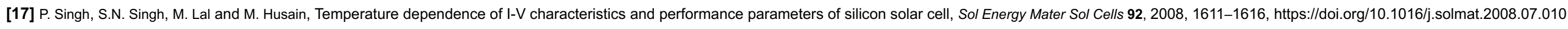

[18] M. Fisac, F.X. Villasevil and A.M. López, High-efficiency photovoltaic technology including thermoelectric generation, J Power Sources 252, 2014, 264-269, https://doi.org/10.1016/j.jpowsour.2013.11.121.

[19] O. Dupré, R. Vaillon and M.A. Green, Thermal behavior of photovoltaic devices, 2017, Springer International Publishinghttps://doi.org/10.1007/978-3-319-49457-9.

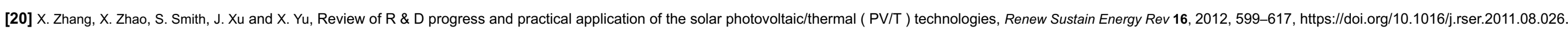

[21] J.F. Li, W.S. Liu, L.D. Zhao and M. Zhou, High-performance nanostructured thermoelectric materials, NPG Asia Mater 2, 2010, 152-158, https://doi.org/10.1038/asiamat.2010.138.

[22] D.M. Rowe, Thermoelectric handbook: macro to nano, 2006, CRC Press, Taylor \& Francis Group.

[23] A.J. Minnich, M.S. Dresselhaus, Z.F. Ren and G. Chen, Bulk nanostructured thermoelectric materials: current research and future prospects, Energy Environ Sci 2, 2009, 466-479, https://doi.org/10.1039/b822664b.

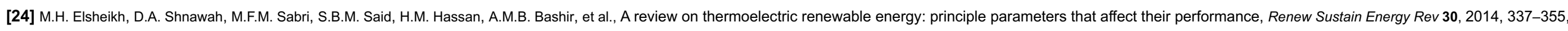
https://doi.org/10.1016/j.rser.2013.10.027.

[25] S.B. Riffat and X. Ma, Improving the coefficient of performance of thermoelectric cooling systems: a review, Int J Energy Res 28, 2004, 753-768, https://doi.org/10.1002/er.991.

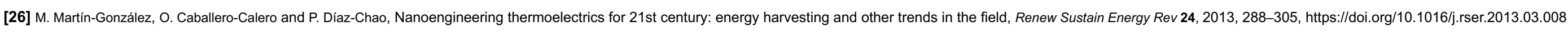

[27] A.R.M. Siddique, S. Mahmud and B. Van Heyst, A review of the state of the science on wearable thermoelectric power generators (TEGs) and their existing challenges, Renew Sustain Energy Rev 73, 2017, 730-744, https://doi.org/10.1016/j.rser.2017.01.177.

[28] X.F. Zheng, C.X. Liu, Y.Y. Yan and Q. Wang, A review of thermoelectrics research - recent developments and potentials for sustainable and renewable energy applications, Renew Sustain Energy Rev 32, 2014, 486-503, https://doi.org/10.1016/j.rser.2013.12.053.

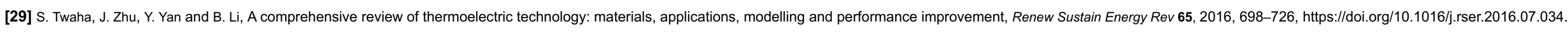

[30] J. Yang, Potential applications of thermoelectric waste heat recovery in the automotive industry, In: Int conf thermoelectr ICT, proc 2005, 2005, 155-159, https://doi.org/10.1109/ICT.2005.1519911. 


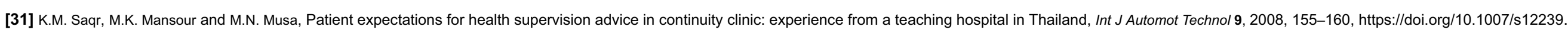

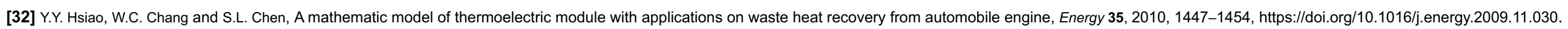

[33] G. Shu, X. Ma, H. Tian, H. Yang, T. Chen and X. Li, Configuration optimization of the segmented modules in an exhaust-based thermoelectric generator for engine waste heat recovery, Energy 160, 2018, 612-624, https://doi.org/10.1016/j.energy.2018.06.175.

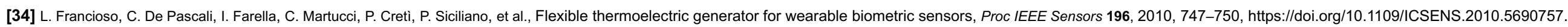

[35] S.J. Kim, J.H. We and B.J. Cho, A wearable thermoelectric generator fabricated on a glass fabric, Energy Environ Sci 7, 2014, 1959-1965, https://doi.org/10.1039/c4ee00242c.

[36] F. Suarez, D.P. Parekh, C. Ladd, D. Vashaee, M.D. Dickey and M.C. Öztürk, Flexible thermoelectric generator using bulk legs and liquid metal interconnects for wearable electronics, Appl Energy 202, 2017, 736-745, https://doi.org/10.1016/j.apenergy.2017.05.181.

[37] S. Qing, A. Rezania, L.A. Rosendahl and X. Gou, Design of flexible thermoelectric generator as human body sensor, Mater Today Proc 5, 2018, 10338-10346, https://doi.org/10.1016/j.matpr.2017.12.282.

[38] R. Amatya and R.J. Ram, Solar thermoelectric generator for micropower applications, J Electron Mater 39, 2010, 1735-1740, https://doi.org/10.1007/s11664-010-1190-8.

[39] D. Madan, Z. Wang, P.K. Wright and J.W. Evans, Printed flexible thermoelectric generators for use on low levels of waste heat, Appl Energy 156, 2015, 587-592, https://doi.org/10.1016/j.apenergy.2015.07.066.

[40] P. Pichanusakorn and P. Bandaru, Nanostructured thermoelectrics, Mater Sci Eng R Rep 67, 2010, 19-63, https://doi.org/10.1016/j.mser.2009.10.001.

[41] S.B. Riffat and X. Ma, Thermoelectrics: a review of present and potential applications, Appl Therm Eng 23, 2003, 913-935, https://doi.org/10.1016/S1359-4311(03)00012-7.

[42] G. Moore and W. Peterson, Solar PV-thermoelectric generator hybrid system: case studies, In: 10th int. Telecommun. Energy conf., san diego, California, USA, 1988, 308-311.

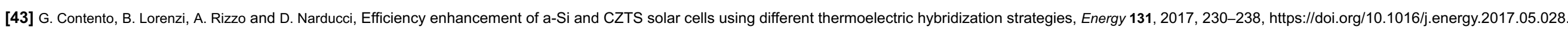

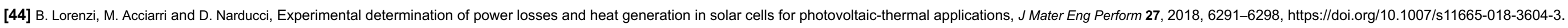

[45] J.R. Howell, R. Siegel and M.P. Mengüç, Thermal radiation heat transfer, fifth ed., 2010, CRC Press, Taylor \& Francis Group; New York.

[46] T.M. Tritt, H. Böttner and L. Chen, Thermoelectrics : direct solar thermal energy conversion, Harnessing Mater Energy 33, 2008, 366-368, https://doi.org/10.1016/0038-092X(80)90311-4.

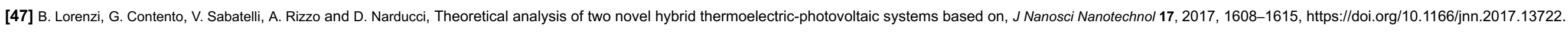

[48] C. Babu and P. Ponnambalam, The role of thermoelectric generators in the hybrid PV/T systems: a review, Energy Convers Manag 151, 2017, 368-385, https://doi.org/10.1016/j.enconman.2017.08.060.

[49] D. Kraemer, L. Hu, A. Muto, X. Chen, G. Chen and M. Chiesa, Photovoltaic-thermoelectric hybrid systems: a general optimization methodology, Appl Phys Lett 92, 2008, 243503, https://doi.org/10.1063/1.2947591.

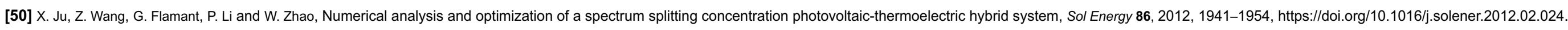

[51] E. Yin, Q. Li and Y. Xuan, A novel optimal design method for concentration spectrum splitting photovoltaicethermoelectric hybrid system, Energy 163, 2018, 519-532, https://doi.org/10.1016/j.apenergy.2018.05.127.

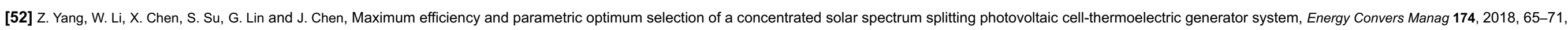
https://doi.org/10.1016/j.enconman.2018.08.038.

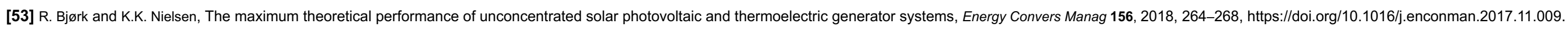

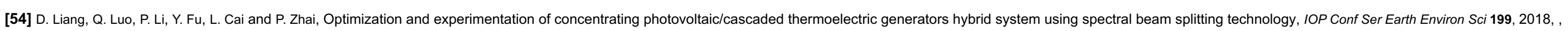
052044https://doi.org/10.1088/1755-1315/199/5/052044.

[55] W.G.J.H.M. Van Sark, Feasibility of photovoltaic - thermoelectric hybrid modules, Appl Energy 88, 2011, 2785-2790, https://doi.org/10.1016/j.apenergy.2011.02.008. 
[56] E. Yin, Q. Li and Y. Xuan, One-day performance evaluation of photovoltaic-thermoelectric hybrid system, Energy 143, 2018, 337-346, https://doi.org/10.1016/j.energy.2017.11.011.

[57] E. Yin, Q. Li and Y. Xuan, Optimal design method for concentrating photovoltaic-thermoelectric hybrid system, Appl Energy 226, 2018, 320-329, https://doi.org/10.1016/j.apenergy.2018.05.127.

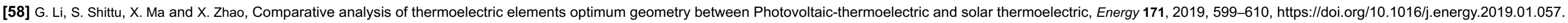

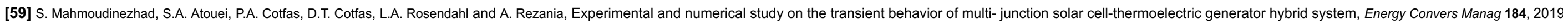
448-455, https://doi.org/10.1016/j.enconman.2019.01.081.

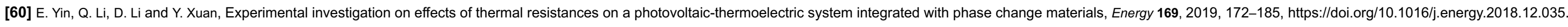

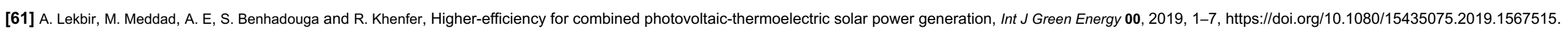

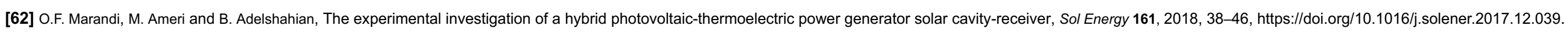

[63] J. Zhang and Y. Xuan, An integrated design of the photovoltaic-thermoelectric hybrid system, Sol Energy 177, 2019, 293-298, https://doi.org/10.1016/j.solener.2018.11.012.

[64] P.M. Rodrigo, A. Valera, E.F. Fernández and F.M. Almonacid, Performance and economic limits of passively cooled hybrid thermoelectric generator-concentrator photovoltaic modules, Appl Energy 238, 2019, 1150-1162, https://doi.org/10.1016/J.APENERGY.2019.01.132.

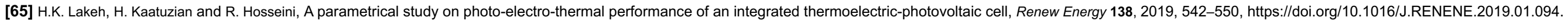

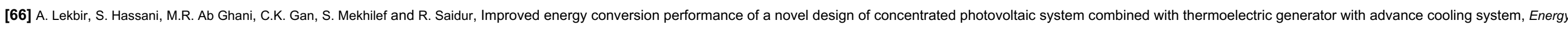
Convers Manag 177, 2018, 19-29, https://doi.org/10.1016/j.enconman.2018.09.053.

[67] B. Lorenzi and G. Chen, Theoretical efficiency of hybrid solar thermoelectric-photovoltaic generators, J Appl Phys 124, 2018, https://doi.org/10.1063/1.5022569.

[68] C. Babu and P. Ponnambalam, The theoretical performance evaluation of hybrid PV-TEG system, Energy Convers Manag 173, 2018, 450-460, https://doi.org/10.1016/j.enconman.2018.07.104.

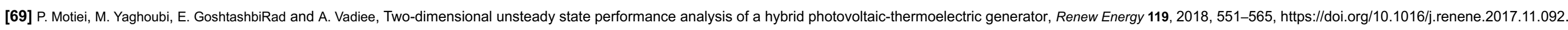

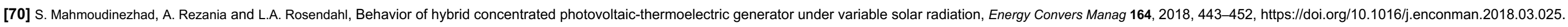

[71] H.R. Fallah Kohan, F. Lotfipour and M. Eslami, Numerical simulation of a photovoltaic thermoelectric hybrid power generation system, Sol Energy 174, 2018, 537-548, https://doi.org/10.1016/j.solener.2018.09.046.

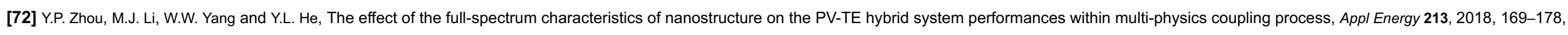
https://doi.org/10.1016/j.apenergy.2018.01.027.

[73] Y. Vorobiev, J. González-Hernández, P. Vorobiev and L. Bulat, Thermal-photovoltaic solar hybrid system for efficient solar energy conversion, Sol Energy 80, 2006, 170-176, https://doi.org/10.1016/j.solener.2005.04.022.

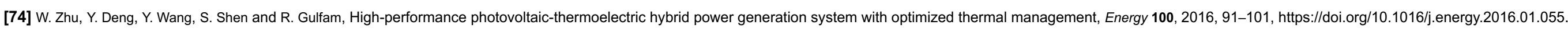

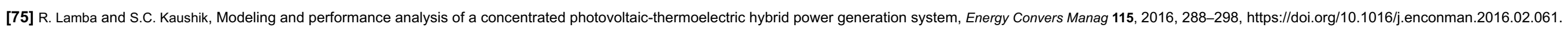

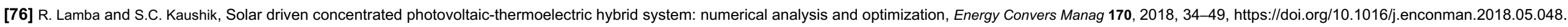

[77] A. Rezania and L.A. Rosendahl, Feasibility and parametric evaluation of hybrid concentrated photovoltaic-thermoelectric system, Appl Energy 187, 2017, 380-389, https://doi.org/10.1016/j.apenergy.2016.11.064.

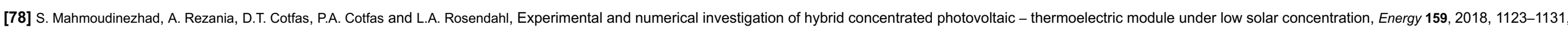
https://doi.org/10.1016/j.energy.2018.06.181.

[79] K.T. Park, S.M. Shin, A.S. Tazebay, H.D. Um, J.Y. Jung, S.W. Jee, et al., Lossless hybridization between photovoltaic and thermoelectric devices, Sci Rep 3, 2013 , 1-6, https://doi.org/10.1038/srep02123. 
[80] B. Lorenzi, M. Acciarri and D. Narducci, Suitability of electrical coupling in solar cell thermoelectric hybridization, Design 2, 2018, 32, https://doi.org/10.3390/designs2030032.

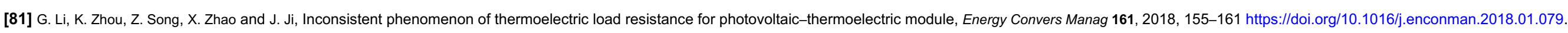

[82] J. Lin, T. Liao and B. Lin, Performance analysis and load matching of a photovoltaic-thermoelectric hybrid system, Energy Convers Manag 105, 2015, 891-899, https://doi.org/10.1016/j.enconman.2015.08.054.

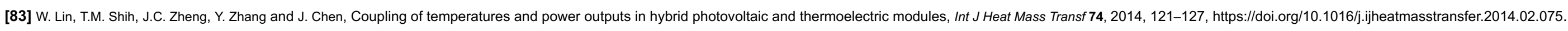

[84] R. Bjørk and K.K. Nielsen, The performance of a combined solar photovoltaic (PV) and thermoelectric generator (TEG) system, Sol Energy 120, 2015, 187-194, https://doi.org/10.1016/j.solener.2015.07.035.

[85] M. Haji, H. Labrim, M. Benaissa, A. Laazizi, H. Ez-Zahraouy, E. Ntsoenzok, et al., Photovoltaic and thermoelectric indirect coupling for maximum solar energy exploitation, Energy Convers Manag 136, 2017, 184-191, https://doi.org/10.1016/j.enconman.2016.12.088.

[86] D. Kraemer, K. McEnaney, M. Chiesa and G. Chen, Modeling and optimization of solar thermoelectric generators for terrestrial applications, Sol Energy 86, 2012, 1338-1350, https://doi.org/10.1016/j.solener.2012.01.025.

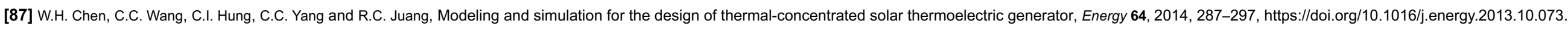

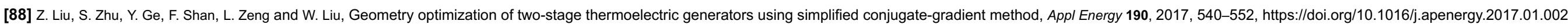

[89] A.S. Al-Merbati, B.S. Yilbas and A.Z. Sahin, Thermodynamics and thermal stress analysis of thermoelectric power generator: influence of pin geometry on device performance, Appl Therm Eng 50, 2013, 683-692, https://doi.org/10.1016/j.applthermaleng.2012.07.021.

[90] H. Hashim, J.J. Bomphrey and G. Min, Model for geometry optimisation of thermoelectric devices in a hybrid PV/TE system, Renew Energy 87, 2016, 458-463, https://doi.org/10.1016/j.renene.2015.10.029.

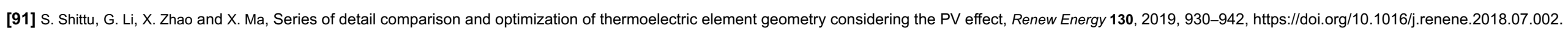

[92] D.N. Kossyvakis, G.D. Voutsinas and E.V. Hristoforou, Experimental analysis and performance evaluation of a tandem photovoltaic-thermoelectric hybrid system, Energy Convers Manag 117, 2016, 490-500, https://doi.org/10.1016/j.enconman.2016.03.023.

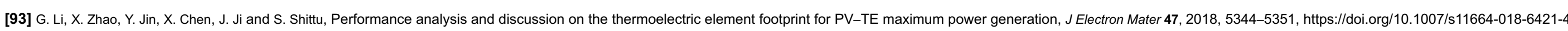

[94] G. Li, X. Chen and Y. Jin, Analysis of the primary constraint conditions of an efficient photovoltaic-thermoelectric hybrid system, Energies 10, 2017, 1-12, https://doi.org/10.3390/en10010020.

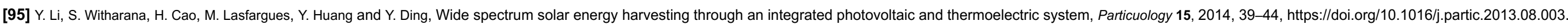

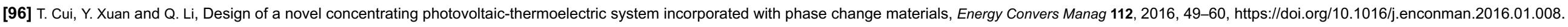

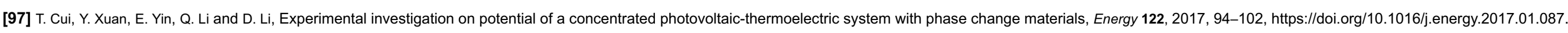

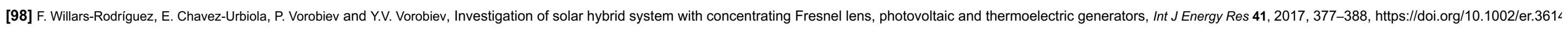

[99] E. Yin, Q. Li and Y. Xuan, Thermal resistance analysis and optimization of photovoltaic-thermoelectric hybrid system, Energy Convers Manag 143, 2017, 188-202, https://doi.org/10.1016/j.enconman.2017.04.004.

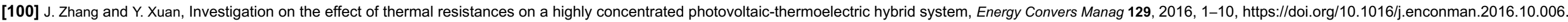

[101] W. Pang, Y. Liu, S. Shao and X. Gao, Empirical study on thermal performance through separating impacts from a hybrid PV/TE system design integrating heat sink, Int Commun Heat Mass Transf 60, 2015, 9-12, https://doi.org/10.1016/j.icheatmasstransfer.2014.11.004.

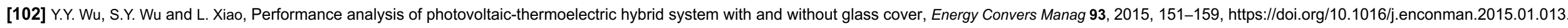

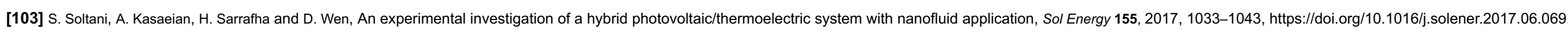

[104] X.Z. Guo, Y.D. Zhang, D. Qin, Y.H. Luo, D.M. Li, Y.T. Pang, et al., Hybrid tandem solar cell for concurrently converting light and heat energy with utilization of full solar spectrum, J Power Sources 195, 2010, 7684-7690, 
https://doi.org/10.1016/j.jpowsour.2010.05.033.

[105] N. Wang, L. Han, H. He, N.-H. Park and K. Koumoto, A novel high-performance photovoltaic-thermoelectric hybrid device, Energy Environ Sci 4, 2011, 3676, https://doi.org/10.1039/c1ee01646f.

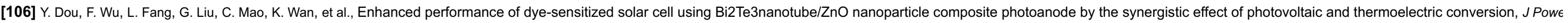
Sources 307, 2016, 181-189, https://doi.org/10.1016/j.jpowsour.2015.12.113

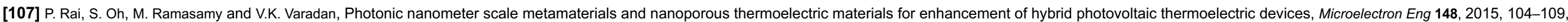
https://doi.org/10.1016/j.mee.2015.09.017.

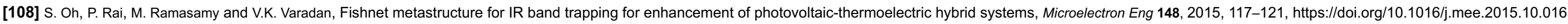

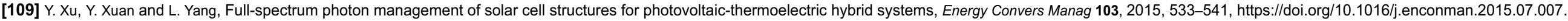

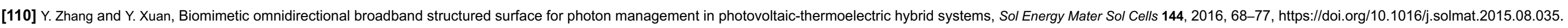

[111] J. Zhang, Y. Xuan and L. Yang, A novel choice for the photovoltaic-thermoelectric hybrid system: the perovskite solar cell, Int J Energy Res 40, 2016, 1400-1409, https://doi.org/10.1002/er.3532.

[112] L. Xu, Y. Xiong, A. Mei, Y. Hu, Y. Rong, Y. Zhou, et al., Efficient perovskite photovoltaic-thermoelectric hybrid device, Adv Energy Mater 8, 2018, 1-5, https://doi.org/10.1002/aenm.201702937.

[113] R. Rabari, S. Mahmud and A. Dutta, Analysis of combined solar photovoltaic-nanostructured thermoelectric generator system, Int J Green Energy 13, 2016, 1175-1184, https://doi.org/10.1080/15435075.2016.1173040.

[114] B.S. Dallan, J. Schumann and F.J. Lesage, Performance evaluation of a photoelectric-thermoelectric cogeneration hybrid system, Sol Energy 118, 2015, 276-285, https://doi.org/10.1016/j.solener.2015.05.034

[115] X. Zhang, K. Chau and C. Chan, Design and implementation of a thermoelectric- photovoltaic hybrid energy source for hybrid electric vehicles, World Electr Veh J 3, 2009 , 271-281.

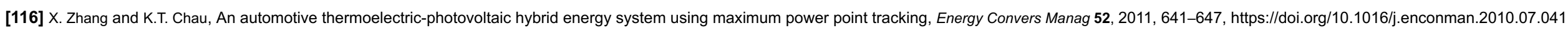

[117] K. Fathima, T.M. Saranya, D. Joseph and A. Kumar, A novel method of utilizing hybrid generator as renewable source, Int J Eng Technol 7, 2016, 1972-1976.

[118] D. Jung, K. Kim and S. Jung, Thermal and solar energy harvesting boost converter with time-multiplexing MPPT algorithm, IEICE Electron Express 13, 2016, 1-9, https://doi.org/10.1109/TEC.2006.874230.

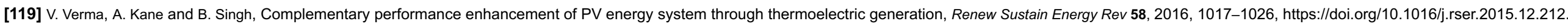

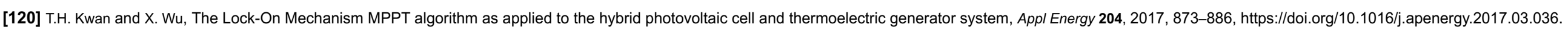

[121] H. Yu, Y. Li, Y. Shang and B. Su, Design and investigation of photovoltaic and thermoelectric hybrid power source for wireless sensor networks, 3rd IEEE Int Conf Nano/Micro Eng Mol Syst NEMS 2008, 196-201, https://doi.org/10.1109/NEMS.2008.4484317.

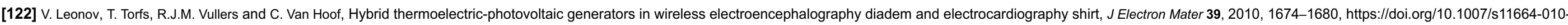
$1230-4$.

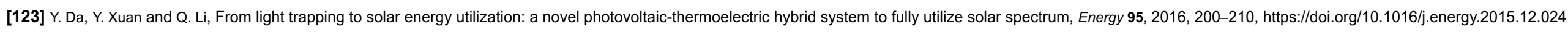

[124] T.H. Kwan and X. Wu, Power and mass optimization of the hybrid solar panel and thermoelectric generators, Appl Energy 165, 2016, 297-307, https://doi.org/10.1016/j.apenergy.2015.12.016.

[125] A. Rezania, D. Sera and L.A. Rosendahl, Coupled thermal model of photovoltaic-thermoelectric hybrid panel for sample cities in Europe, Renew Energy 99, 2016, 127-135, https://doi.org/10.1016/j.renene.2016.06.045.

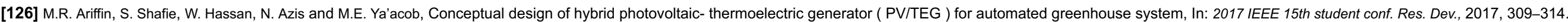

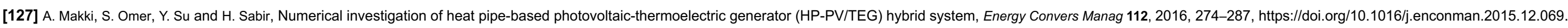

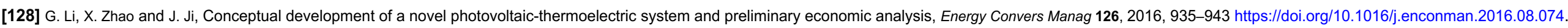


[129] P. Huen and W.A. Daoud, Advances in hybrid solar photovoltaic and thermoelectric generators, Renew Sustain Energy Rev 72, 2017, 1295-1302, https://doi.org/10.1016/j.rser.2016.10.042.

[130] D.L. Chubb and B.S. Good, A combined thermophotovoltaic-thermoelectric energy converter, Sol Energy 159, 2018, 760-767, https://doi.org/10.1016/j.solener.2017.11.030.

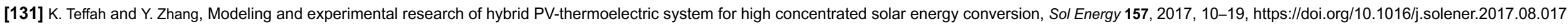

[132] K. Teffah, Y. Zhang and X. Mou, Modeling and experimentation of new thermoelectric cooler-thermoelectric generator module, Energies 11, 2018, 576, https://doi.org/10.3390/en11030576.

[133] K. Yoshikawa, H. Kawasaki, W. Yoshida, T. Irie, K. Konishi, K. Nakano, et al., Silicon heterojunction solar cell with interdigitated back contacts for a photoconversion efficiency over $26 \%$, Nat Energy 2, $2017,17032$.

[134] J. Benick, A. Richter, R. Müller, H. Hauser, F. Feldmann, P. Krenckel, et al., High-efficiency n-type HP mc silicon solar cells, IEEE J Photovoltaics 7, 2017, 1171-1175, https://doi.org/10.1109/JPHOTOV.2017.2714139.

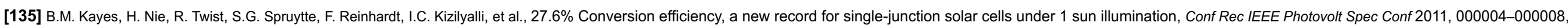
https://doi.org/10.1109/PVSC.2011.6185831.

[136] M. Wanlass, Systems and methods for advanced ultra-high-performance, InP solar cells 9 (590), 2017, 131 B2.

[137] Solar Frontier Press Release, Solar frontier achieves world record thin-film solar cell efficiency of 22.9\%, 20 December 2017 http://www.solar-frontier.com/eng/news/2017/1220_press.html, Accessed 10 November 2018.

[138] J.-L. Wu, Y. Hirai, T. Kato, H. Sugimoto and V. Bermudez, New world record efficiency up to $22.9 \%$ for Cu(in,Ga)(Se,S)2 thin-film solar cells, 2018.

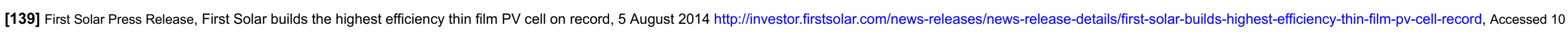
November 2018.

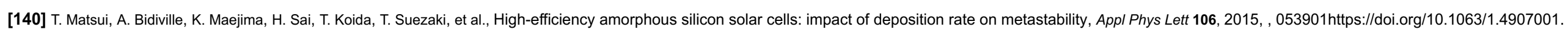

[141] W.S. Yang, J.H. Noh, N.J. Jeon, Y.C. Kim, S. Ryu, J. Seo, et al., High-performance photovoltaic perovskite layers fabricated through intramolecular exchange, Science 348 (80-), 2015, 1234-1237, doi:10/f7fzcf.

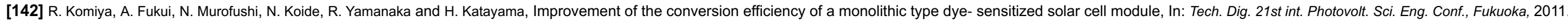

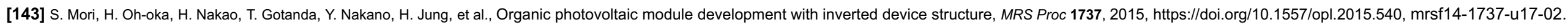

[144] M. Mizoshiri, M. Mikami and K. Ozaki, Thermal-photovoltaic hybrid solar generator using thin-film thermoelectric modules, Jpn J Appl Phys 51, 2012, 2-7, https://doi.org/10.1143/JJAP.51.06FL07.

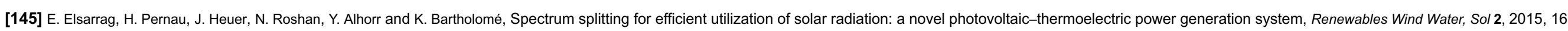
https://doi.org/10.1186/s40807-015-0016-y.

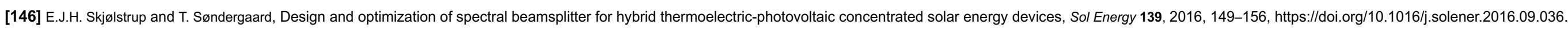

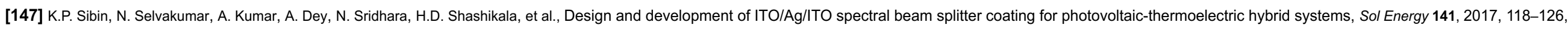
https://doi.org/10.1016/j.solener.2016.11.027.

[148] Mustofa, Z. Djafar, Syafaruddin and W.H. Piarah, A new hybrid of photovoltaic-thermoelectric generator with hot mirror as spectrum splitter, J Phys Sci 29, 2018, 63-75, https://doi.org/10.21315/jps2018.29.s2.6.

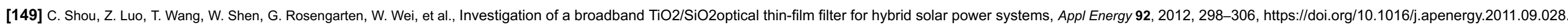

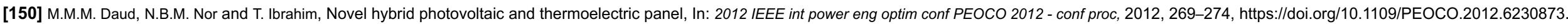

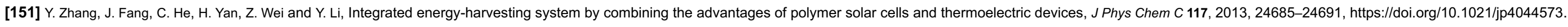

[152] J. Zhang, Y. Xuan and L. Yang, Performance estimation of photovoltaic-thermoelectric hybrid systems, Energy 78, 2014, 895-903, https://doi.org/10.1016/j.energy.2014.10.087.

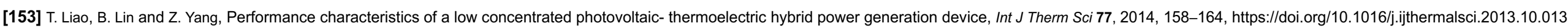


[154] H. Chen, N. Wang and H. He, Equivalent circuit analysis of photovoltaic - thermoelectric hybrid device with different TE module structure, Adv Condens Matter Phys 2014, 1-16, https://doi.org/10.1155/2014/824038.

[155] O. Beeri, O. Rotem, E. Hazan, E.A. Katz, A. Braun and Y. Gelbstein, Hybrid photovoltaic-thermoelectric system for concentrated solar energy conversion: experimental realization and modeling, J Appl Phys 118, 2015, , 115104https://doi.org/10.1063/1.4931428.

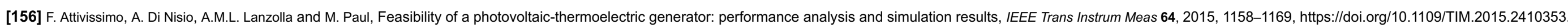

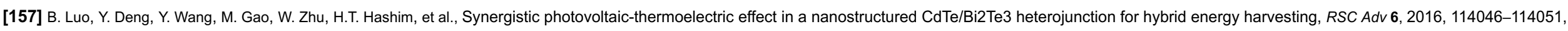
https://doi.org/10.1039/C6RA20149K.

[158] W. Pang, H. Yu, Y. Zhang and H. Yan, Electrical characteristics of a hybrid photovoltaic/thermoelectric generator system, Energy Techno/ 6, 2017, 1248-1254, https://doi.org/10.1002/ente.201700801.

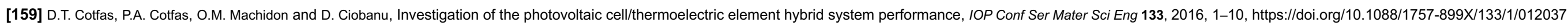

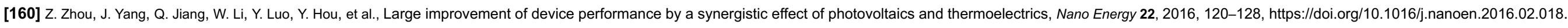

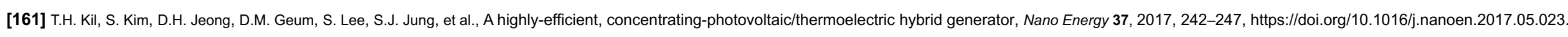

[162] D. Li, Y. Xuan, Q. Li and H. Hong, Exergy and energy analysis of photovoltaic-thermoelectric hybrid systems, Energy 126, 2017, 343-351, https://doi.org/10.1016/j.energy.2017.03.042.

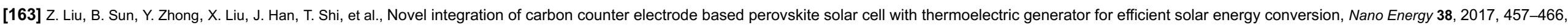
https://doi.org/10.1016/j.nanoen.2017.06.016.

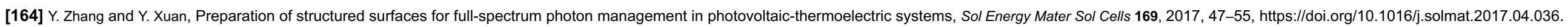

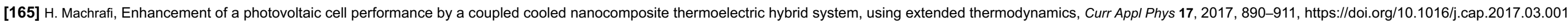

[166] Y. Jeyashree, P.B. Hepsiba, S. Indirani, A.D. Savio and Y. Sukhi, Solar energy harvesting using hybrid photovoltaic and thermoelectric generating system, Glob J Pure Appl Math 13, 2017 , 5935-5944.

[167] Y. Nishijima, R. Komatsu, T. Yamamura, G. Seniutinas and J. Saulius, Design concept of a hybrid photo-voltaic/thermal conversion cell for mid-infrared light energy harvester, Opt Mater Express 7, 2017, 3484-3493, https://doi.org/10.4161/19336918.2014.994893.

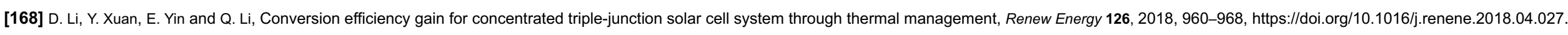

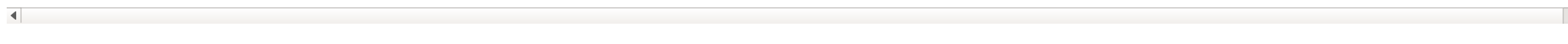

Highlights

- A detailed review of photovoltaic thermal management is presented

- The role of thermoelectric generator in hybrid photovoltaic enhancement is explained.

- Research focus areas in hybrid photovoltaic/thermoelectric generator are discussed.

- Recommendations for future research on hybrid PV/TEG are presented.

\section{Queries and Answers}

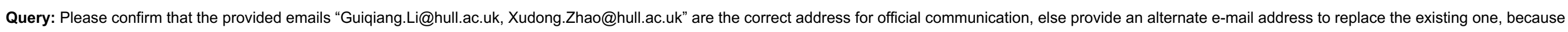
private e-mail addresses should not be used in articles as the address for communication. 
Answer: Yes, the provided emails are correct for official communication.

Query: Please note that 'Fig.23, Fig.24' was/were not cited in the text. Please check that the citation(s) suggested by the copyeditor are in the appropriate place, and correct if necessary. Answer: No, the citations suggested are not in the appropriate place. An instruction on their right location has been given.

Query: Have we correctly interpreted the following funding source(s) and country names you cited in your article: Innovate UK, United Kingdom; EPSRC, United Kingdom? Answer: Yes

Query: Please confirm that given names and surnames have been identified correctly and are presented in the desired order and please carefully verify the spelling of all authors' names. Answer: Yes

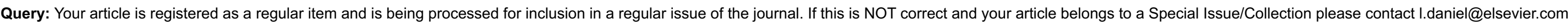
immediately prior to returning your corrections.

Answer: Yes this is correct. 